\title{
Periodic cyclic homology of reductive $p$-adic groups
}

\author{
Maarten Solleveld
}

\begin{abstract}
Let $G$ be a reductive $p$-adic group, $\mathscr{H}(G)$ its Hecke algebra and $S(G)$ its Schwartz algebra. We will show that these algebras have the same periodic cyclic homology. This provides an alternative proof of the Baum-Connes conjecture for $G$, modulo torsion.

As preparation for our main theorem we prove two results that have independent interest. Firstly, a general comparison theorem for the periodic cyclic homology of finite type algebras and certain Fréchet completions thereof. Secondly, a refined form of the Langlands classification for $G$, which clarifies the relation between the smooth spectrum and the tempered spectrum.
\end{abstract}

Mathematics Subject Classification (2000). 20G25, 16E40.

Keywords. Periodic cyclic homology, $p$-adic groups, Baum-Connes conjecture, finite type algebras, topological K-theory.

\section{Contents}

1 Comparison theorems for periodic cyclic homology . . . . . . . . . . . 506

1.1 Finite type algebras . . . . . . . . . . . . . . . . 506

1.2 The commutative case . . . . . . . . . . . . . . . . . . 508

1.3 Comparison with topological K-theory . . . . . . . . . . . . . . 512

1.4 The general case . . . . . . . . . . . . . . . . . . . . 514

2 Some representation theory of reductive $p$-adic groups $\ldots \ldots$. . . . . . . . . 519

2.1 Convolution algebras . . . . . . . . . . . . . . . . . . 519

2.2 The Plancherel theorem . . . . . . . . . . . . . . . . . . 525

2.3 The Langlands classification . . . . . . . . . . . . . . . . . . . . 529

2.4 Parametrizing irreducible representations . . . . . . . . . . . . . . . . 533

3 The noncommutative geometry of reductive $p$-adic groups . . . . . . . 537

3.1 Periodic cyclic homology . . . . . . . . . . . . . . . 537

3.2 Example: $\mathrm{SL}_{2}\left(\mathbb{Q}_{p}\right) \ldots \ldots \ldots \ldots \ldots \ldots \ldots$

3.3 Equivariant cosheaf homology . . . . . . . . . . . . . . . . 547

3.4 The Baum-Connes conjecture . . . . . . . . . . . . . . . . . . . . . . . . 549

References . . . . . . . . . . . . . . . . . . . . 555 


\section{Introduction}

In this article we compare different homological invariants of algebras associated to reductive $p$-adic groups. Group algebras, or more precisely convolution algebras of functions on groups, have always been important objects of study in noncommutative geometry. Generally speaking the idea (or hope) is that the interaction between representation theory, harmonic analysis, operator algebras and geometry leads to results that can not (yet) be proven inside only one of these areas.

By definition a group algebra encodes information about a group, so its homological invariants should reflect properties of the group. Therefore, whenever one considers two convolution algebras associated to the same group, their invariants should be closely related. Yet in practice this has to be taken with quite a few grains of salt. For example the periodic cyclic homology of $\mathbb{C}\left[\mathbb{Z} \rtimes C_{2}\right]$ is isomorphic to the de Rham cohomology (with complex coefficients) of the disjoint union of $\mathbb{C}^{\times} /\left(z \sim z^{-1}\right)$ and a point. On the other hand the periodic cyclic homology of the group- $C^{*}$-algebra $C^{*}\left(\mathbb{Z} \rtimes C_{2}\right)$ does not give any new information: it is the algebra itself in even degrees and it vanishes in odd degrees. So finding a meaningful invariant of the group is a matter of both choosing the right group algebra and the right functor.

For Fréchet algebras topological K-theory is a good choice since it is a very stable functor. It has the excision property and is invariant under homotopy equivalences and under passing to holomorphically closed dense subalgebras. Comparing with the above example, the $\mathrm{K}$-theory of $C^{*}\left(\mathbb{Z} \rtimes C_{2}\right)$ is again isomorphic to the cohomology of a manifold. But the manifold has been adjusted to its compact form

$$
S^{1} /\left(z \sim z^{-1}\right) \cup \text { point } \cong[-1,1] \cup \text { point }
$$

and we must take its singular cohomology with integral coefficients. We remark that subalgebras consisting of all functions on $\mathbb{Z} \rtimes C_{2}$ with rapid (resp. subexponential) decay have the same K-theory.

Nevertheless it can be hard to compute a $K$-group of a lesser known algebra. Indeed in the classical picture of $K_{0}$ one has to find all homotopy classes of projectors, a task for which no general procedure exists.

Of course there is a wider choice of interesting functors. Arguably the most subtle one is Hochschild homology $\left(\mathrm{HH}_{*}\right)$, the oldest homology theory for algebras. Depending on the circumstances it can be regarded as group cohomology, (noncommutative) differential forms or as a torsion functor. Moreover Hochschild homology can be computed with the very explicit bar complex. On the other hand $\mathrm{HH}_{*}$ does neither have the excision property nor is it homotopy invariant.

We mainly discuss periodic cyclic homology $\left(\mathrm{HP}_{*}\right)$ in this article. Although it carries less information than Hochschild homology, it is much more stable. The relation between $\mathrm{HH}_{*}$ and $\mathrm{HP}_{*}$ is analogous to that between differential forms and de Rham cohomology, as the Hochschild-Kostant-Rosenberg theorem makes explicit in the case of smooth commutative algebras. It is known that periodic cyclic homology has 
the excision property and is invariant under Morita equivalences, diffeotopy equivalences and nilpotent extensions. Together with the link to Hochschild homology these make $\mathrm{HP}_{*}$ computable in many cases. This functor works especially well on the category of finite type algebras [KNS], that is, algebras that are finitely generated modules over the coordinate ring of some complex affine variety. In this category an important principle holds for periodic cyclic homology, namely that it depends only on the primitive ideal spectrum of the algebra in question.

A similar principle fails miserably for topological algebras, even for commutative ones. For example let $M$ be a compact smooth manifold. Then $\mathrm{HP}_{*}\left(C^{\infty}(M)\right)$ is the de Rham cohomology of $M$, while $\mathrm{HP}_{*}(C(M))$ just returns the $C^{*}$-algebra $C(M)$. The underlying reason is that $\mathrm{HP}_{*}$ does not only see the (irreducible) modules of an algebra, it also takes the derived category into account. In geometric terms this means that $\mathrm{HP}_{*}(A)$ does not only depend on the primitive ideal spectrum of $A$ as a topological space but also on the structure of the "infinitesimal neighborhoods" of points in this space. These infinitesimal neighborhoods are automatically right for finite type algebras, because they can be derived from the underlying affine variety. But the spectrum of $C(M)$ does not admit infinitesimal neighborhoods. Indeed, these have to be related to the powers of a maximal ideal $I$, but they collapse because $\overline{I^{n}}=I$ for all $n \in \mathbb{N}$.

We remark that this problem can partially be overcome with a clever variation on $\mathrm{HP}_{*}$, local cyclic homology [Mey3]. This functor gives nice results for $C^{*}$ algebras because it is stable under isoradial homomorphisms of complete bornological algebras. On the other hand this theory does require an array of new techniques.

We will add a new move under which periodic cyclic homology is invariant. Let $\Gamma$ be a finite group acting (by $\alpha$ ) on a nonsingular complex affine variety $X$, and suppose that we have a cocycle $u: \Gamma \rightarrow \mathrm{GL}_{N}(\mathcal{O}(X))$. Then $\alpha$ and $u$ combine to an action of $\Gamma$ on $M_{N}(\mathcal{O}(X))$ :

$$
\gamma \cdot f=u_{\gamma} f^{\alpha(\gamma)} u_{\gamma}^{-1}
$$

The algebra of $\Gamma$-invariants $M_{N}(\mathcal{O}(X))^{\Gamma}$ has a natural Fréchet completion, namely $M_{N}\left(C^{\infty}(X)\right)^{\Gamma}$. We will show in Section 1 that the inclusion map induces an isomorphism

$$
\operatorname{HP}_{*}\left(M_{N}(\mathcal{O}(X))^{\Gamma}\right) \rightarrow \operatorname{HP}_{*}\left(M_{N}\left(C^{\infty}(X)\right)^{\Gamma}\right) .
$$

The proof is based on abelian filtrations of both algebras, that is, on sequences of ideals such that the successive quotients are Morita equivalent to commutative algebras. In terms of primitive ideal spectra this means that we have stratifications of finite length such that all the strata are Hausdorff spaces.

Let us discuss these general issues in connection with reductive $p$-adic groups. We use this term as an abbreviation of "the $\mathbb{F}$-rational points of a connected reductive algebraic group, where $\mathbb{F}$ is a non-Archimedean local field". Such groups are important in number theory, especially in relation with the Langlands program. There are many open problems for reductive $p$-adic groups, for example there is no definite 
classification of irreducible smooth representations. There are two general strategies to divide the classification problem into pieces, thereby reducing it to either supercuspidal or square-integrable representations.

First we start with a supercuspidal representation of a Levi-component of a parabolic subgroup of our given group $G$. Then we apply parabolic induction to obtain a (not necessarily irreducible) smooth $G$-representation. The collection of representations obtained in this way contains every irreducible object at least once.

The second method involves the Langlands classification, which reduces the problem to the classification of irreducible tempered $G$-representations. These can be found as in the first method, replacing supercuspidal by square-integrable representations. This kind of induction was studied in [ScZi]. The procedure yields a collection of (possibly decomposable) tempered $G$-representations, in which every irreducible tempered representation appears at least once.

Our efforts in Section 2 result in a refinement of the Langlands classification. To every irreducible smooth $G$-representation we associate a quadruple $(P, A, \omega, \chi)$ consisting of a parabolic pair $(P, A)$, a square-integrable representation $\omega$ of the Levi component $Z_{G}(A)$ and an unramified character $\chi$ of $Z_{G}(A)$. Moreover we prove that this quadruple is unique up to $G$-conjugacy. This result is useful for comparing the smooth spectrum of $G$ with its tempered spectrum and for constructing stratifications of these spectra.

Let us consider three convolution algebras associated to a reductive $p$-adic group $G$. Firstly the reduced $C^{*}$-algebra $C_{r}^{*}(G)$, secondly the Hecke algebra $\mathscr{H}(G)$ and thirdly Harish-Chandra's Schwartz algebra $\delta(G)$. For each of these algebras we will study the most appropriate homology theory. For the reduced $C^{*}$-algebra this is topological K-theory, and for the Hecke algebra we take periodic cyclic homology. For the Schwartz algebra the choice is more difficult. Since it is not a Fréchet algebra the usual versions of K-theory are not even defined for $S(G)$. It is not difficult to give an ad-hoc definition, and the natural ways to do so quickly lead to $K_{*}(\delta(G)) \cong K_{*}\left(C_{r}^{*}(G)\right)$. Nevertheless, we would also like to compute the periodic cyclic homology of $S(G)$. It is definitely not a good idea to do this with respect to the algebraic tensor product, because that would ignore the topology on $\delta(G)$. As explained in [Mey2], $\delta(G)$ is best regarded as a bornological algebra, and therefore we will study its periodic cyclic homology with respect to the completed bornological tensor product $\hat{\otimes}_{\mathbb{C}}$.

That this is the right choice is vindicated by two comparison theorems. On the one hand it is already proved in [Sol1] that the Chern character for $\delta(G)$ induces an isomorphism

$$
\operatorname{ch} \otimes \mathrm{id}: K_{*}\left(C_{r}^{*}(G)\right) \otimes_{\mathbb{Z}} \mathbb{C} \rightarrow \operatorname{HP}_{*}\left(\delta(G), \hat{\otimes}_{\mathbb{C}}\right) .
$$

On the other hand we will show in Section 3.1 that the inclusion of $\mathscr{H}(G)$ in $\delta(G)$ induces an isomorphism

$$
\mathrm{HP}_{*}(\mathscr{H}(G)) \rightarrow \mathrm{HP}_{*}\left(S(G), \hat{\otimes}_{\mathbb{C}}\right) .
$$


Of course both comparison theorems can be decomposed as direct sums over the Bernstein components of $G$. The proof of (4) is an extension of the ideas leading to (2) and is related to the following quote [SSZ], p. 3:

The remarkable picture which emerges is that Bernstein's decomposition of $\mathcal{M}(G)$ into its connected components refines into a stratification of $\mathscr{Y}(G)$ where the strata, at least up to nilpotent elements, are module categories over commutative rings. We strongly believe that such a picture holds true for any group $G$.

If this is indeed the case then our methods can be applied to many other groups.

The most important application of (3) and (4) lies in their relation with yet other invariants of $G$. Let $\beta G$ be the affine Bruhat-Tits building of $G$. The classical paper $[\mathrm{BCH}]$ introduced among others the equivariant $K$-homology $K_{*}^{G}(\beta G)$ and the cosheaf homology $\mathrm{CH}_{*}^{G}(\beta G)$. Let us recall the known relations between these invariants. The Baum-Connes conjecture for $G$, proven by Lafforgue [Laf], asserts that the assembly map

$$
\mu: K_{*}^{G}(\beta G) \rightarrow K_{*}\left(C_{r}^{*}(G)\right)
$$

is an isomorphism. Voigt [Voi3] constructed a Chern character

$$
\operatorname{ch}: K_{*}^{G}(\beta G) \rightarrow \mathrm{CH}_{*}^{G}(\beta G),
$$

which becomes an isomorphism after tensoring the left-hand side with $\mathbb{C}$. Furthermore it is already known from [HiNi] that $\mathrm{CH}_{*}^{G}(\beta G)$ is isomorphic to $\mathrm{HP}_{*}(\mathscr{H}(G))$. Altogether we get a diagram

$$
\begin{array}{ccc}
K_{*}^{G}(\beta G) \otimes_{\mathbb{Z}} \mathbb{C} & \cong K_{*}\left(C_{r}^{*}(G)\right) \otimes_{\mathbb{Z}} \mathbb{C} \\
\cong & \cong \\
\mathrm{CH}_{*}^{G}(\beta G) & \cong & \operatorname{HP}_{*}(\mathscr{H}(G)),
\end{array}
$$

whose existence was already conjectured in [BHP3]. We will prove in Section 3.3 that it commutes. The four isomorphisms all have mutually independent proofs, so any three of them can be used to proof the fourth. None of the proofs is easy, but it seems to the author that (5) is the most difficult one. Therefore it is not unreasonable to say that this diagram provides an alternative way to prove the Baum-Connes conjecture for reductive $p$-adic groups, modulo torsion.

Returning to our initial broad point of view, we conclude that we used representation theory and harmonic analysis to prove results in noncommutative geometry. It is outlined in [BHP3] how cosheaf homology could be used to prove representation theoretic results. The author hopes that the present article might contribute to the understanding of the issues raised in [BHP3]. 


\section{Comparison theorems for periodic cyclic homology}

1.1. Finite type algebras. We will compare the periodic cyclic homology of certain finite type algebras and completions thereof. The motivating example of the result we aim at is as follows.

Let $X$ be a nonsingular complex affine variety. We consider the algebras $\mathcal{O}(X)$ of regular (polynomial) functions and $C^{\infty}(X)$ of complex valued smooth functions on $X$. By default, if we talk about continuous or differentiable functions on $X$ or about the cohomology of $X$, we always do this with respect to the analytic topology on $X$, obtained from embedding $X$ in a complex affine space.

The Hochschild-Kostant-Rosenberg-Connes theorem tells us what the periodic cyclic homology of these algebras looks like:

$$
\begin{aligned}
\operatorname{HP}_{n}(\mathcal{O}(X)) & \cong \bigoplus_{m \in \mathbb{Z}} H_{D R}^{n+2 m}(X ; \mathbb{C}), \\
\operatorname{HP}_{n}\left(C^{\infty}(X)\right) & \cong \bigoplus_{m \in \mathbb{Z}} H_{D R}^{n+2 m}(X ; \mathbb{C}) .
\end{aligned}
$$

In the first line we must take the de Rham cohomology of $X$ as an algebraic variety. However, according to a result of Grothendieck and Deligne this is naturally isomorphic to the de Rham cohomology of $X$ as a smooth manifold. Hence the inclusion $\mathcal{O}(X) \rightarrow C^{\infty}(X)$ induces an isomorphism

$$
\mathrm{HP}_{*}(\mathcal{O}(X)) \rightarrow \operatorname{HP}_{*}\left(C^{\infty}(X)\right) .
$$

Now let us discuss this in greater generality, allowing noncommutative algebras. We denote the primitive ideal spectrum of any algebra $A$ by $\operatorname{Prim}(A)$ and we endow it with the Jacobson topology, which is the natural noncommutative generalization of the Zariski topology. An algebra homomorphism $\phi: A \rightarrow B$ is called spectrum preserving if it induces a bijection on primitive ideal spaces, in the following sense. For every $J \in \operatorname{Prim}(B)$ there is a unique $I \in \operatorname{Prim}(A)$ such that $\phi^{-1}(J) \subset I$, and the map $\operatorname{Prim}(B) \rightarrow \operatorname{Prim}(A): J \mapsto I$ is bijective.

Since we do not want to get too far away from commutative algebras, we will work with finite type algebras, see [KNS], [BaNi]. Let $\mathbf{k}$ be the ring of regular functions on some complex affine variety. A finite type $\mathbf{k}$-algebra is a $\mathbf{k}$-algebra that is finitely generated as a $\mathbf{k}$-module. The periodic cyclic homology of a finite type algebra always has finite dimension, essentially because this is case for $\mathcal{O}(X)$ [KNS], Theorem 1. Moreover it depends only on the primitive ideal spectrum of the algebra, in the following sense:

Theorem 1.1 ([BaNi], Theorem 8). A spectrum preserving morphism of finite type k-algebras induces an isomorphism on periodic cyclic homology .

Morally speaking $\operatorname{HP}_{*}(A)$ should correspond to the "cohomology" of $\operatorname{Prim}(A)$. However, this is only a nonseparated scheme, so classical cohomology theories will not do. Yet this can be made precise with sheaf cohomology [Sol3], Section 2.2. 
It is not unreasonable to expect that there is always some Fréchet completion $A_{\text {smooth }}$ of $A=A_{\text {alg }}$ such that the inclusion $A_{\text {alg }} \rightarrow A_{\text {smooth }}$ induces an isomorphism

$$
\mathrm{HP}_{*}\left(A_{\text {alg }}\right) \rightarrow \mathrm{HP}_{*}\left(A_{\text {smooth }}\right) .
$$

A good candidate appears to be

$$
A_{\text {smooth }}=A_{\text {alg }} \otimes_{\mathcal{O}(X)} C^{\infty}(X)
$$

if the center of $A_{\text {alg }}$ is $\mathcal{O}(X)$. However I believe that it would be rather cumbersome to determine precisely under which conditions this works out. Moreover I do not know whether the resulting smooth algebras are interesting in this generality. Therefore we restrict our attention to algebras of a specific (but still rather general) form, which we will now describe.

Let $\Gamma$ be a finite group acting (by $\alpha$ ) on the nonsingular complex affine variety $X$. Take $N \in \mathbb{N}$ and consider the algebra of matrix-valued regular functions on $X$ :

$$
\mathcal{O}\left(X ; M_{N}(\mathbb{C})\right):=M_{N}(\mathcal{O}(X))=\mathcal{O}(X) \otimes M_{N}(\mathbb{C}) .
$$

Suppose that we have elements $u_{\gamma} \in \mathrm{GL}_{N}(\mathcal{O}(X))$ such that

$$
(\gamma \cdot f)(x)=u_{\gamma}(x) f\left(\alpha_{\gamma}^{-1} x\right) u_{\gamma}^{-1}(x)
$$

defines a group action of $\Gamma$ on $M_{N}(\mathcal{O}(X))$, by algebra homomorphisms. We do not require that $\gamma \mapsto u_{\gamma}$ is a group homomorphism. Nevertheless the above does imply that there exists a 2 -cocycle $\lambda: \Gamma \times \Gamma \rightarrow \mathcal{O}(X)^{\times}$such that

$$
u_{\gamma}\left(u_{\gamma^{\prime}} \circ \alpha_{\gamma}^{-1}\right)=\lambda\left(\gamma, \gamma^{\prime}\right) u_{\gamma \gamma^{\prime}} .
$$

In particular, for every $x \in X$ we get a projective $\Gamma_{x}$-representation

$$
\left(\pi_{x}, \mathbb{C}^{N}\right) \text { with } \pi_{x}(\gamma)=u_{\gamma}(x) .
$$

The element $u_{\gamma}$ should be regarded as an intertwiner between representations with $\mathcal{O}(X)$-characters $x$ and $\alpha_{\gamma}(x)$. We are interested in the finite type algebra

$$
A_{\text {alg }}=\mathcal{O}\left(X ; M_{N}(\mathbb{C})\right)^{\Gamma}
$$

of $\Gamma$-invariant elements. We note that restriction of a module from $A_{\text {alg }}$ to $\mathcal{O}(X)^{\Gamma}$ defines a continuous finite to one surjection [KNS], Lemma 1,

$$
\theta: \operatorname{Prim}\left(A_{\mathrm{alg}}\right) \rightarrow X / \Gamma \text {. }
$$

Examples. Classical algebras of this type are

$$
\begin{aligned}
\mathcal{O}(X)^{\Gamma} & =\mathcal{O}(X / \Gamma), \\
\mathcal{O}(X ; \operatorname{End}(\mathbb{C}[\Gamma]))^{\Gamma} & \cong \mathcal{O}(X) \rtimes \Gamma .
\end{aligned}
$$


For example, take $X=\mathbb{C}^{3}$ and $\Gamma=\mathbb{Z} / 3 \mathbb{Z}$, acting through cyclic permutations of the coordinates. Put $A_{\text {alg }}=\mathcal{O}(X) \rtimes \Gamma$. Almost all points $\Gamma x \in X / \Gamma$ correspond to a unique irreducible $A_{\text {alg }}$-module, namely $\operatorname{Ind}_{\mathcal{O}(X)}^{A_{\text {alg }}} \mathbb{C}_{x}$. Only the points $(z, z, z)$ with $z \in \mathbb{C}$ carry three irreducible $\mathcal{O}(X) \rtimes \Gamma$-modules, of the form $\mathbb{C}_{(z, z, z)} \otimes \mathbb{C}_{\zeta}$ with $\zeta$ a cubic root of unity.

More generally, suppose that we have a larger group $G$ with a normal subgroup $N$ such that $\Gamma=G / N$. Let $(\pi, V)$ be a $G$-representation on which $N$ acts by a character. Then

$$
(g \cdot f)(x)=\pi(g) f\left(\alpha_{g N}^{-1} x\right) \pi\left(g^{-1}\right)
$$

defines an action of $G$ on $\mathcal{O}(X ; \operatorname{End}(V))$ which factors through $\Gamma$, so

$$
\mathcal{O}(X ; \operatorname{End}(V))^{G}=\mathcal{O}(X ; \operatorname{End}(V))^{\Gamma} .
$$

If we put $u_{\gamma}=\pi(g)$ for some $g$ with $g N=\gamma$ then we are in the setting of (1.6). Yet in general there is no canonical choice for $u_{\gamma}$, and we end up with a nontrivial cocycle $\lambda$. (In fact this a typical example of a projective $\Gamma$-representation.)

The natural Fréchet completion of (1.8) is

$$
A_{\text {smooth }}=C^{\infty}\left(X ; M_{N}(\mathbb{C})\right)^{\Gamma} .
$$

This algebra has the same spectrum as $A_{\text {alg }}$, but the two algebras induce different Jacobson topologies on this set. The Jacobson topology from $A_{\text {smooth }}$ is finer and makes Prim $\left(A_{\text {smooth }}\right)$ a non-Hausdorff manifold. (By this we mean a second countable topological space in which every point has a neighborhood that is homeomorphic to $\mathbb{R}^{n}$.)

The map (1.3) is an isomorphism in the special cases where $A_{\text {alg }}$ is as in (1.10) and $A_{\text {smooth }}$ as in (1.11), as follows from comparing [KNS] and [Was]. We will show that it holds much more generally, for example if we take $A_{\text {smooth }}=C^{\infty}\left(X^{\prime} ; M_{N}(\mathbb{C})\right)^{\Gamma}$ with $X^{\prime}$ a suitable deformation retract of $X$. Such an algebra is finitely generated as a $C^{\infty}\left(X^{\prime}\right)^{\Gamma}$-module, and therefore we will call it a (topological) finite type algebra.

1.2. The commutative case. Let $\mathrm{CC}_{* *}(A)$ be the periodic cyclic bicomplex associated to an algebra $A$ [Lod], Section 1. Its terms are of the form $A^{\otimes n}$ and its homology is by definition $\mathrm{HP}_{*}(A)$. For topological algebras we must specify which particular topological tensor product we wish to use in cyclic theory. By default we work with a Fréchet algebra $A$ whose topology is defined by submultiplicative seminorms, and with the completed projective tensor product $\hat{\otimes}$. Since this is a completion of the algebraic tensor product we get natural maps

$$
\begin{aligned}
\mathrm{CC}_{* *}(A, \otimes) & \rightarrow \mathrm{CC}_{* *}(A, \hat{\otimes}), \\
\operatorname{HP}_{*}(A, \otimes) & \rightarrow \operatorname{HP}_{*}(A, \hat{\otimes}) .
\end{aligned}
$$


We abbreviate $\operatorname{CC}_{* *}(A)=\mathrm{CC}_{* *}(A, \hat{\otimes})$ and $\operatorname{HP}_{*}(A)=\operatorname{HP}_{*}(A, \hat{\otimes})$ for Fréchet algebras. Recall that an extension of topological algebras is admissible if it is split exact in the category of topological vector spaces. An ideal $I$ of $A$ is admissible if $0 \rightarrow I \rightarrow A \rightarrow A / I \rightarrow 0$ is admissible.

Any extension $0 \rightarrow A \rightarrow B \rightarrow C \rightarrow 0$ gives rise to a short exact sequence of differential complexes

$$
0 \rightarrow \mathrm{CC}_{* *}(B, A) \rightarrow \mathrm{CC}_{* *}(B) \rightarrow \mathrm{CC}_{* *}(C) \rightarrow 0
$$

with $\mathrm{CC}_{* *}(B, A):=\operatorname{ker}\left(\mathrm{CC}_{* *}(B) \rightarrow \mathrm{CC}_{* *}(C)\right)$. By a standard construction in homological algebra this leads to a long exact sequence

$$
\cdots \rightarrow \mathrm{HP}_{i}(B, A) \rightarrow \mathrm{HP}_{i}(B) \rightarrow \mathrm{HP}_{i}(C) \rightarrow \mathrm{HP}_{i+1}(B, A) \rightarrow \cdots .
$$

Actually this sequence wraps up to an exact hexagon since $\mathrm{HP}_{i+2} \cong \mathrm{HP}_{i}$. The inclusion $\mathrm{CC}_{* *}(A) \rightarrow \mathrm{CC}_{* *}(B, A)$ induces a map $\mathrm{HP}_{*}(A) \rightarrow \mathrm{HP}_{*}(B, A)$. One of our main tools will be the excision property of periodic cyclic homology:

Theorem 1.2. Let $0 \rightarrow A \rightarrow B \rightarrow C \rightarrow 0$ be an extension of (nontopological) algebras or an admissible extensions of Fréchet algebras. Then $\mathrm{HP}_{*}(A) \rightarrow \mathrm{HP}_{*}(B, A)$ is an isomorphism, and (1.12) yields an exact hexagon

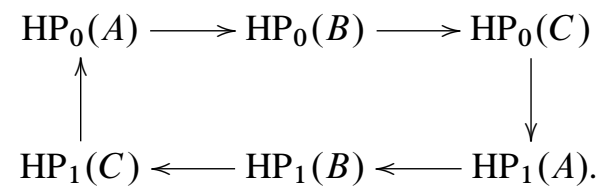

Proof. The basic version of this theorem is due to Wodzicki [Wod]. It was proved in general by Cuntz and Quillen [CuQu], [Cun].

Suppose that we want to prove that an algebra homomorphism $\phi: A \rightarrow B$ induces an isomorphism on $\mathrm{HP}_{*}$. The excision property can be used as follows.

Lemma 1.3. Suppose that there are sequences of ideals

$$
A=I_{0} \supset I_{1} \supset \cdots \supset I_{d}=0, \quad B=J_{0} \supset J_{1} \supset \cdots \supset J_{d}=0
$$

with the properties

- $\phi\left(I_{p}\right) \subset J_{p}$ for all $p \geq 0$,

- $\operatorname{HP}_{*}\left(I_{p-1} / I_{p}\right) \rightarrow \mathrm{HP}_{*}\left(J_{p-1} / J_{p}\right)$ is an isomorphism for all $p \geq 0$,

- if $B$ (respectively $A$ ) is Fréchet then the ideals $J_{p}$ (respectively $I_{p}$ ) are admissible.

Then $\mathrm{HP}_{*}(\phi): \mathrm{HP}_{*}(A) \rightarrow \mathrm{HP}_{*}(B)$ is an isomorphism. 
Proof. Left as an exercise. Use the five lemma.

Generally speaking a good tool to compute the periodic cyclic homology of a finite type algebra $A$ is a filtration by ideals $I_{p}$ such that the successive quotients $I_{p-1} / I_{p}$ behave like commutative algebras. In particular Prim $\left(I_{p-1} / I_{p}\right)$ should be a (separated) affine variety, so this gives rise a kind of stratification of $\operatorname{Prim}(A)$. This can be formalized with the notion of an abelian filtration [KNS].

To describe suitable smooth analogues of $A_{\text {alg }}$ we must say what precisely we mean by smooth functions on spaces that are not manifolds. Let $Z \subset Y$ be subsets of a smooth manifold $X$ and let $V$ be a complete topological vector space. Define

$$
\begin{aligned}
C^{\infty}(Y ; V) & :=\left\{f: Y \rightarrow V \mid \text { there exist an open } U \subset X \text { and } \tilde{f} \in C^{\infty}(U ; V)\right. \\
\text { such that } \left.Y \subset U,\left.\tilde{f}\right|_{Y}=f\right\}, & \\
C_{0}^{\infty}(Y, Z) & :=\left\{f \in C^{\infty}(Y ; \mathbb{C})|f|_{Z}=0\right\}, \\
C_{0}^{\infty}(Y, Z ; V) & :=\left\{f \in C^{\infty}(Y ; V)|f|_{Z}=0\right\} .
\end{aligned}
$$

Recall that a corner in a manifold is a point that has a neighborhood homeomorphic to $\mathbb{R}^{n} \times[0, \infty)^{m}$, with $m>0$. To apply excision we will often need the following result of Tougeron.

Theorem 1.4 ([Tou], Théorème IX.4.3). Let $Y$ be a smooth manifold and $Z$ a smooth submanifold, both possibly with corners. The following extension is admissible:

$$
0 \rightarrow C_{0}^{\infty}(Y, Z) \rightarrow C^{\infty}(Y) \rightarrow C^{\infty}(Z) \rightarrow 0 .
$$

For completeness we include an extended version of the Hochschild-KostantRosenberg theorem for periodic cyclic homology. We abbreviate

$$
H^{[n]}(Y)=\bigoplus_{m \in \mathbb{Z}} \check{H}^{n+2 m}(Y ; \mathbb{C}) .
$$

Theorem 1.5. Let $Y$ be a smooth manifold, possibly noncompact and with corners. There is a natural isomorphism

$$
\operatorname{HP}_{*}\left(C^{\infty}(Y)\right) \cong H^{[*]}(Y) .
$$

Proof. For $Y$ compact and without boundary this is due to Connes [Con], p. 130, who in fact proved the much stronger statement

$$
\mathrm{HH}_{*}\left(C^{\infty}(Y)\right) \cong \Omega^{*}(Y) .
$$

Here $\mathrm{HH}_{*}$ denotes Hochschild homology and $\Omega^{*}$ means differential forms with complex values. By Corollary 4.3 and Theorem 7.1 of [BLT], (1.13) still holds if $Y$ is allowed to have corners and may be noncompact. Hence $\mathrm{HP}_{*}\left(C^{\infty}(?)\right)$ and $H^{[*]}($ ?) agree at least locally. By Theorems 1.2 and 1.4 both these functors satisfy excision, so they agree on every manifold. 
Let $Y$ be a complex affine variety and $Z$ a closed subvariety, both possibly reducible and singular. In line with the above we write

$$
\begin{aligned}
\mathcal{O}_{0}(Y, Z) & :=\left\{f \in \mathcal{O}(Y)|f|_{Z}=0\right\}, \\
\mathcal{O}_{0}(Y, Z ; V) & :=O_{0}(Y, Z) \otimes V .
\end{aligned}
$$

Let $\breve{H}^{*}(Y, Z ; \mathbb{C})$ denote the Čech cohomology of the pair $(Y, Z)$, with complex coefficients and with respect to the analytic topology. Because $\mathrm{HP}_{*}$ and $K_{*}$ have a $\mathbb{Z} / 2 \mathbb{Z}$-grading, it is convenient to impose this also on Čech cohomology. Therefore we write

$$
H^{[n]}(Y, Z):=\bigoplus_{m \in \mathbb{Z}} \check{H}^{n+2 m}(Y, Z ; \mathbb{C})
$$

Now we are ready to state and prove the comparison theorem for the periodic cyclic homology of commutative algebras.

Theorem 1.6. a) There is a natural isomorphism $\operatorname{HP}_{*}\left(\mathcal{O}_{0}(Y, Z)\right) \cong H^{[*]}(Y, Z)$.

b) Suppose that $Y \backslash Z$ is nonsingular and that $\widetilde{C}_{0}^{\infty}(Y, Z)$ is a Fréchet algebra with the properties

- $\mathcal{O}_{0}(Y, Z) \subset \widetilde{C}_{0}^{\infty}(Y, Z) \subset C_{0}^{\infty}(Y, Z)$,

- if the partial derivatives of $f \in C_{0}^{\infty}(Y, Z)$ all vanish on $Z$ then $f \in \widetilde{C}_{0}^{\infty}(Y, Z)$.

Then $\mathrm{HP}_{*}\left(\mathcal{O}_{0}(Y, Z)\right) \rightarrow \mathrm{HP}_{*}\left(\widetilde{C}_{0}^{\infty}(Y, Z)\right)$ is an isomorphism.

Proof. a) was proved in [KNS], Theorem 9.

b) By assumption $Y \backslash Z$ with the analytic topology is a smooth manifold. Let $N$ be a closed neighborhood of $Z$ in $Y$ such that $Y \backslash N$ is a smooth manifold and a deformation retract of $Y \backslash Z$. Let $r:[0,1] \times Y \rightarrow Y$ be a smooth map with the properties

(1) $r_{1}=\operatorname{id}_{Y}$ where $r_{t}(y):=r(t, y)$,

(2) $r_{t}(z)=z$ for all $z \in Z, t \in[0,1]$,

(3) $r_{t}(N) \subset N$ for all $t \in[0,1]$ and $r_{0}(N)=Z$,

(4) $r_{t}^{-1}(Z)$ is a neighborhood of $Z$ for all $t<1$,

(5) $r_{t}: r_{t}^{-1}(Y \backslash Z) \rightarrow Y \backslash Z$ is a diffeomorphism for all $t \in[0,1]$.

Consider the algebra homomorphisms

$$
\begin{aligned}
& C_{0}^{\infty}(Y, N) \rightarrow \widetilde{C}_{0}^{\infty}(Y, Z), \quad f \mapsto f, \\
& \widetilde{C}_{0}^{\infty}(Y, Z) \rightarrow C_{0}^{\infty}(Y, N), \quad f \mapsto f \circ r_{0} .
\end{aligned}
$$

By construction these are diffeotopy equivalences. The diffeotopy invariance of $\mathrm{HP}_{*}(?, \hat{\otimes})[\mathrm{Con}]$, p. 125 , ensures that

$$
\mathrm{HP}_{*}\left(\widetilde{C}_{0}^{\infty}(Y, Z)\right) \cong \mathrm{HP}_{*}\left(C_{0}^{\infty}(Y, N)\right)
$$


Write $\tilde{Y}=r_{1 / 2}^{-1}(Y \backslash Z)$ and $\tilde{N}=N \cap \tilde{Y}$. This could look like

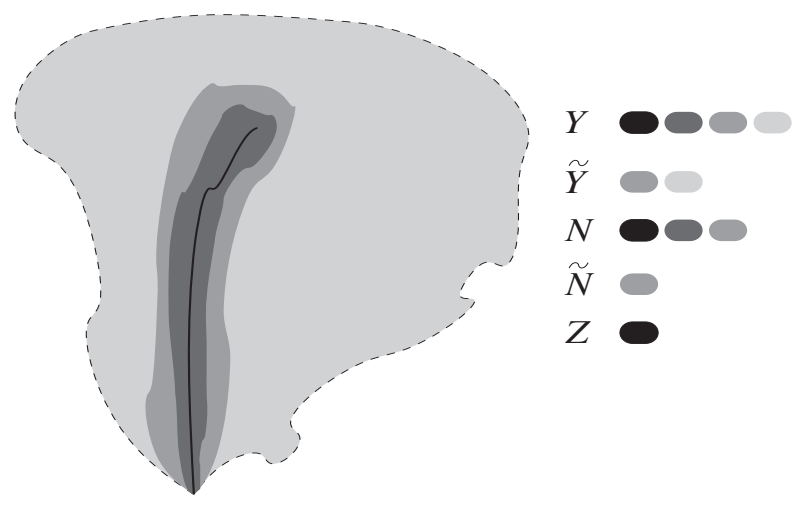

By Theorem 1.4 there is an admissible extension

$$
0 \rightarrow C_{0}^{\infty}(Y, N)=C_{0}^{\infty}(\tilde{Y}, \tilde{N}) \rightarrow C^{\infty}(\tilde{Y}) \rightarrow C^{\infty}(\tilde{N}) \rightarrow 0 .
$$

Combining this with Theorems 1.2 and 1.5 yields natural isomorphisms

$$
\operatorname{HP}_{*}\left(C^{\infty}(\tilde{Y}, \tilde{N})\right) \cong H^{[*]}(\tilde{Y}, \tilde{N}) \cong H^{[*]}(Y, N) \cong H^{[*]}(Y, Z) .
$$

Now consider the diagram

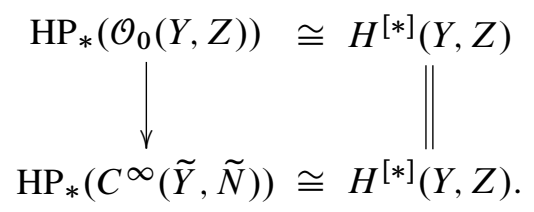

It commutes by naturality, so the arrow is an isomorphism.

1.3. Comparison with topological K-theory. Let $\Gamma$ be a finite group which acts by diffeomorphisms on a smooth manifold $X$. We will frequently meet algebras of the form

$$
C_{0}^{\infty}\left(Y, Z ; M_{N}(\mathbb{C})\right)^{\Gamma},
$$

where $Y$ and $Z$ are $\Gamma$-stable closed submanifolds of $X$. We allow our manifolds to have corners (and in particular a boundary), since these appear naturally in orbifolds. But we must be careful, because the algebra $C^{\infty}(X)^{\Gamma}$ of smooth functions on the orbifold $X / \Gamma$ does not contain all smooth functions on the manifold $X / \Gamma$. Namely, there are some conditions for the partial derivatives of $f \in C^{\infty}(X)^{\Gamma}$ at the corners.

This makes it rather tricky to compute the periodic cyclic homology of algebras like (1.15). Actually such algebras would look much better if we could replace smooth functions by continuous functions, because continuous functions are not bothered by 
mild singularities like corners. But then another problem pops up, that $\mathrm{HP}_{*}$ tends to give tautological results for Banach algebras. For example, if $K$ is any compact Hausdorff space then

$$
\begin{aligned}
& \operatorname{HP}_{0}(C(K))=C(K), \\
& \operatorname{HP}_{1}(C(K))=0 .
\end{aligned}
$$

To overcome these inconveniences we will compute the periodic cyclic homology of (1.3) via its topological K-theory. The K-theory of Fréchet algebras was defined by Phillips [Phi]. As is well known, these theories are related by a Chern character $\mathrm{ch}: K_{*} \rightarrow \mathrm{HP}_{*}$.

Theorem 1.7 ([Nis], Theorem 16). Let $0 \rightarrow A \rightarrow B \rightarrow C \rightarrow 0$ be an extension of Fréchet algebras. The various Chern characters form a commutative diagram

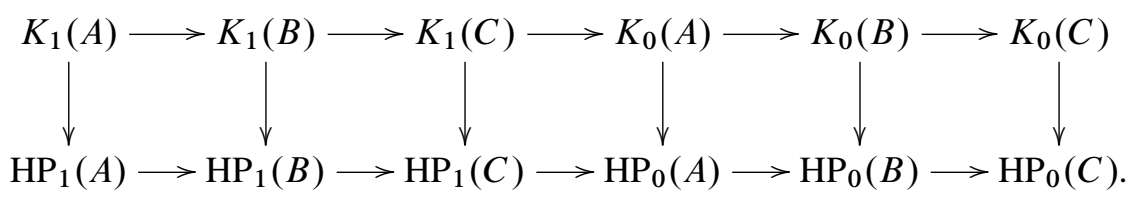

If the extension is admissible and $\eta: K_{0}(C) \rightarrow K_{1}(A)$ and $\partial: \operatorname{HP}_{0}(C) \rightarrow \operatorname{HP}_{1}(A)$ denote the connecting maps, then $\mathrm{ch} \circ \eta=2 \pi i \partial \circ \mathrm{ch}$.

Let $\mathcal{L} \mathcal{I}$ be the class of Fréchet algebras $A$ for which

$$
\operatorname{ch} \otimes \operatorname{id}_{\mathbb{C}}: K_{*}(A) \otimes_{\mathbb{Z}} \mathbb{C} \rightarrow \mathrm{HP}_{*}(A) \otimes_{\mathbb{C}} \mathbb{C}=\mathrm{HP}_{*}(A)
$$

is an isomorphism.

Corollary 1.8. Let $0 \rightarrow A \rightarrow B \rightarrow C \rightarrow 0$ be an admissible extension of Fréchet algebras. If two of $A, B, C$ belong to the class CIA, then so does the third.

Proof. This follows from Theorems 1.2 and 1.7, in combination with Bott periodicity and the five lemma.

The class CIA is very large since it is also closed under finite direct products, tensoring with $M_{n}(\mathbb{C})$ and diffeotopy equivalences. Furthermore all topological finite type algebras are in $\mathcal{L} \mathcal{L}$ :

Theorem 1.9. Let $\Gamma$ be a finite group acting (by $\alpha)$ on a smooth manifold $X$ and let $u_{\gamma} \in \mathrm{GL}_{N}\left(C^{\infty}(X)\right)$ be elements such that

$$
\gamma \cdot f=u_{\gamma}\left(f \circ \alpha_{\gamma}^{-1}\right) u_{\gamma}^{-1}
$$

defines an action of $\Gamma$ on $C^{\infty}\left(X ; M_{N}(\mathbb{C})\right)$. Let $Z \subset Y$ be $\Gamma$-stable submanifolds of $X$, possibly with corners. Then $C_{0}^{\infty}\left(Y, Z ; M_{N}(\mathbb{C})\right)^{\Gamma}$ belongs to the class $\mathcal{C} \mathcal{I} \mathcal{A}$. 
Proof. According to Theorem 1.4,

$$
0 \rightarrow C_{0}^{\infty}(X, Y) \rightarrow C^{\infty}(X) \rightarrow C^{\infty}(Y) \rightarrow 0
$$

is an admissible extension. Hence so is

$$
0 \rightarrow C_{0}^{\infty}\left(X, Y ; M_{N}(\mathbb{C})\right) \rightarrow C_{0}^{\infty}\left(X, Z ; M_{N}(\mathbb{C})\right) \rightarrow C_{0}^{\infty}\left(Y, Z ; M_{N}(\mathbb{C})\right) \rightarrow 0
$$

Because $\Gamma$ is finite the same holds for the subalgebras of $\Gamma$-invariants in (1.17) and (1.18). Together with Corollary 1.8 this reduces the proof to the case $Z=\emptyset$.

Thus we have to show that $C^{\infty}\left(Y ; M_{N}(\mathbb{C})\right)^{\Gamma}$ is in $\mathcal{E} \mathcal{L} \mathcal{A}$. Except for a detail this is the content of [Sol1], Theorem 6. The small complication is that in [Sol1] the author considered only $\Gamma$-manifolds $Y$ without corners, because according to [Ill] those have smooth equivariant triangulations. However, $Y$ admits such a triangulation even if it has corners, because it is embedded in the smooth $\Gamma$-manifold $X$.

1.4. The general case. First we discuss a motivating concept for our comparison theorem. Suppose that $\Gamma$ acts on $\mathbb{Z}^{n}$ and consider the tori

$$
\begin{aligned}
X & :=\operatorname{Hom}_{\mathbb{Z}}\left(\mathbb{Z}^{n}, \mathbb{C}^{\times}\right) \cong\left(\mathbb{C}^{\times}\right)^{n} \cong \operatorname{Prim}\left(\mathbb{C}\left[\mathbb{Z}^{n}\right]\right), \\
X^{\prime} & :=\operatorname{Hom}_{\mathbb{Z}}\left(\mathbb{Z}^{n}, S^{1}\right) \cong\left(S^{1}\right)^{n} \cong \operatorname{Prim}\left(\delta\left(\mathbb{Z}^{n}\right)\right),
\end{aligned}
$$

where the $S$ stands for complex valued Schwartz functions. We want to compare the periodic cyclic homology of the algebras

$$
\begin{aligned}
A_{\text {alg }} & =\mathbb{C}\left[\mathbb{Z}^{n}\right] \rtimes \Gamma=\mathcal{O}(X) \rtimes \Gamma, \\
A_{\text {smooth }} & =S\left(\mathbb{Z}^{n}\right) \rtimes \Gamma=C^{\infty}\left(X^{\prime}\right) \rtimes \Gamma .
\end{aligned}
$$

Although these algebras definitely have different spectra, it is natural to expect that $\mathrm{HP}_{*}\left(A_{\text {alg }}\right) \cong \mathrm{HP}_{*}\left(A_{\text {smooth }}\right)$. The best notion to explain this appears to be "diffeotopy equivalence of non-Hausdorff spaces". This is a typically noncommutative geometric concept that might contradict one's intuition. The idea is that $\operatorname{Prim}\left(A_{\text {alg }}\right)$ and $\operatorname{Prim}\left(A_{\text {smooth }}\right)$ are equivalent in this specific sense, and for that very reason these algebras have the same periodic cyclic homology.

Since usual homotopies do not see non-Hausdorff phenomena we have to be careful in defining this notion. We say that a continuous map $X \rightarrow Y$ is a homotopy (diffeotopy) equivalence of non-Hausdorff spaces if there exist finite length stratifications of $X$ and $Y$ such that

- all the strata are Hausdorff spaces,

- the maps are compatible with the stratifications,

- the induced maps on the strata are homotopy (diffeotopy) equivalences.

Notice that we do not require the existence of a continuous map from $Y$ to $X$ because that would exclude many interesting cases. For example consider the plane with a 
doubled origin. It is contractible in the usual sense, but as a non-Hausdorff space it is diffeotopy equivalent to two points!

Generally speaking an algebra homomorphism that induces a diffeotopy equivalence on primitive ideal spectra (endowed with a suitable "analytic" topology) should yield an isomorphism on periodic cyclic homology. Our notion is probably not strong enough to prove things with, but does provide a generalization of Theorem 1.1 at a conceptual level.

Thus inspired we require the following conditions for our comparison theorem. Let $\Gamma$ be a finite group acting (by $\alpha$ ) on a nonsingular complex affine variety $X$. Suppose that we have elements $u_{\gamma} \in \mathrm{GL}_{N}(\mathcal{O}(X))$ such that

$$
\gamma \cdot f=u_{\gamma}\left(f \circ \alpha_{\gamma}^{-1}\right) u_{\gamma}^{-1}
$$

defines an action of $\Gamma$ on the algebra $\mathcal{O}\left(X ; M_{N}(\mathbb{C})\right)$. Let $X^{\prime}$ be a submanifold of $X$ with the following properties:

- $X^{\prime}$ is smooth but may have corners,

- $X^{\prime}$ is stable under the action of $\Gamma$,

- the inclusion $X^{\prime} \rightarrow X$ is a diffeotopy equivalence in the category of smooth $\Gamma$-manifolds.

We write

$$
A_{\text {alg }}=\mathcal{O}\left(X ; M_{N}(\mathbb{C})\right)^{\Gamma}, \quad A_{\text {smooth }}=C^{\infty}\left(X^{\prime} ; M_{N}(\mathbb{C})\right)^{\Gamma} .
$$

The inclusion of $\operatorname{Prim}\left(A_{\text {smooth }}\right)$ in $\operatorname{Prim}\left(A_{\text {alg }}\right)$ is the prototype of a diffeotopy equivalence of non-Hausdorff spaces.

Theorem 1.10. The natural map $A_{\mathrm{alg}} \rightarrow A_{\mathrm{smooth}}$ induces an isomorphism

$$
\mathrm{HP}_{*}\left(A_{\text {alg }}\right) \rightarrow \mathrm{HP}_{*}\left(A_{\text {smooth }}\right) .
$$

Proof. We will use Lemma 1.3 to reduce the proof to manageable pieces. For every subset $H \subset \Gamma$ the variety $X^{H}$ is nonsingular and $X^{\prime H}=X^{H} \cap X^{\prime}$ is a submanifold. Let $\mathscr{L}$ be the collection of all the irreducible components of all the $X^{H}$, with $H$ running over all subsets of $\Gamma$. Let $\mathscr{L}_{p}$ be its subset of elements of dimension $\leq p$ and define $\Gamma$-stable closed subvarieties

$$
X_{p}:=\bigcup_{V \in \mathscr{L}_{p}} V .
$$

By the third condition above $X_{p}^{\prime}:=X_{p} \cap X^{\prime}$ is $\Gamma$-equivariantly diffeotopy equivalent to $X_{p}$.

By construction the singularities of $X_{p}$ are all contained in $X_{p-1}$. Moreover, because the action of $\Gamma$ is locally linearizable, these singularities are all normal 
crossings. Hence we have for arbitrary subsets $G, H \subset \Gamma$ :

$$
\begin{aligned}
X^{G} \cap X^{H} & =X^{G \cup H}, \\
\mathcal{O}_{0}\left(X^{G} \cup X^{H}, X^{G} \cap X^{H}\right) & =\mathcal{O}_{0}\left(X^{G}, X^{G \cup H}\right) \oplus \mathcal{O}_{0}\left(X^{H}, X^{G \cup H}\right), \\
X^{\prime G} \cap X^{\prime H} & =X^{\prime G \cup H}, \\
C_{0}^{\infty}\left(X^{\prime G} \cup X^{\prime H}, X^{\prime G} \cap X^{\prime H}\right) & =C_{0}^{\infty}\left(X^{\prime G}, X^{\prime G \cup H}\right) \oplus C_{0}^{\infty}\left(X^{\prime H}, X^{\prime G \cup H}\right) .
\end{aligned}
$$

Consider the sequences of ideals

$$
\begin{aligned}
A_{\text {alg }} & =I_{0} \supset I_{1} \supset \cdots \supset I_{\operatorname{dim} X}=0, \\
A_{\text {smooth }} & =J_{0} \supset J_{1} \supset \cdots \supset J_{\operatorname{dim} X}=0, \\
I_{p} & =\left\{a \in A_{\text {alg }}|a|_{X_{p}}=0\right\}=\mathcal{O}_{0}\left(X, X_{p} ; M_{N}(\mathbb{C})\right)^{\Gamma}, \\
J_{p} & =\left\{a \in A_{\text {smooth }}|a|_{X_{p}^{\prime}}=0\right\}=C_{0}^{\infty}\left(X^{\prime}, X_{p}^{\prime} ; M_{N}(\mathbb{C})\right)^{\Gamma} .
\end{aligned}
$$

We want to compare the periodic cyclic homology of the quotients

$$
I_{p-1} / I_{p} \cong \mathcal{O}_{0}\left(X_{p}, X_{p-1} ; M_{N}(\mathbb{C})\right)^{\Gamma}, \quad J_{p-1} / J_{p} \cong C_{0}^{\infty}\left(X_{p}^{\prime}, X_{p-1}^{\prime} ; M_{N}(\mathbb{C})\right)^{\Gamma} .
$$

Let $Z(B)$ denote the center of an algebra $B$. To the filtration (1.20) we associate the spaces

$$
Y_{p}=\operatorname{Prim}\left(Z\left(A_{\mathrm{alg}} / I_{p}\right)\right), \quad Z_{p}=\left\{I \in Y_{p} \mid Z\left(I_{p-1} / I_{p}\right) \subset I\right\} .
$$

The $Y_{p}$ are called the centers of the filtration and the $Z_{p}$ the subcenters. Notice that, unlike Prim $\left(I_{p}\right)$, these are separated algebraic varieties. By $[\mathrm{KNS}]$, Theorem 9, there are natural isomorphisms

$$
\mathrm{HP}_{*}\left(Z\left(I_{p-1} / I_{p}\right)\right) \cong H^{[*]}\left(Y_{p}, Z_{p}\right) \cong \operatorname{HP}_{*}\left(\mathcal{O}_{0}\left(Y_{p}, Z_{p}\right)\right) .
$$

We claim that

$$
Z\left(I_{p} / I_{p-1}\right) \rightarrow I_{p} / I_{p-1}
$$

is a spectrum preserving morphism of finite type $\mathcal{O}(X)$-algebras. To see this, we first consider the composite map

$$
\theta_{p}: \operatorname{Prim}\left(I_{p-1} / I_{p}\right) \rightarrow \operatorname{Prim}\left(Z\left(I_{p} / I_{p-1}\right)\right)=Y_{p} \backslash Z_{p} \rightarrow\left(X_{p} \backslash X_{p-1}\right) / \Gamma .
$$

For $x \in X_{p} \backslash X_{p-1}$ the image of

$$
I_{p-1} / I_{p} \rightarrow M_{N}(\mathbb{C}): f \mapsto f(x)
$$

is the semisimple algebra $S_{x}=\operatorname{End}_{\pi_{x}\left(\Gamma_{x}\right)}\left(\mathbb{C}^{N}\right)$, where $\pi_{x}(\gamma)=u_{\gamma}(x)$. Since $u_{\gamma} \in \mathrm{GL}_{N}(\mathcal{O}(X))$ the type of $\left(\pi_{x}, \mathbb{C}^{N}\right)$ as a projective $\Gamma_{x}$-representation cannot change along the irreducible components of $X^{\Gamma_{x}}$. Together with (1.20) this implies 
that, locally on $\left(X_{p} \backslash X_{p-1}\right) / \Gamma, I_{p-1} / I_{p}$ is of the form $S_{x} \otimes \mathcal{O}_{0}\left(U, U^{\prime}\right)$. Hence $Z\left(I_{p-1} / I_{p}\right)$ is locally of the form $Z\left(S_{x}\right) \otimes \mathcal{O}_{0}\left(U, U^{\prime}\right)$, which proves our claim about (1.23). Now we may apply Theorem 1.1 , which tells us that

$$
\mathrm{HP}_{*}\left(Z\left(I_{p-1} / I_{p}\right)\right) \rightarrow \mathrm{HP}_{*}\left(I_{p-1} / I_{p}\right)
$$

is an isomorphism.

According to a very general extension theorem for smooth functions [BiSc], Theorem 0.2.1, the ideals $J_{p}$ are admissible in $A_{\text {smooth }}$. Alternatively, this can be derived from Theorem 1.4 using (1.19). From (1.19) we also see that $J_{p-1} / J_{p}$ is a finite direct sum of algebras of the form considered in Theorem 1.9, so

$$
\text { ch } \otimes \mathrm{id}: K_{*}\left(J_{p-1} / J_{p}\right) \otimes_{\mathbb{Z}} \mathbb{C} \rightarrow \operatorname{HP}_{*}\left(J_{p-1} / J_{p}\right)
$$

is an isomorphism. Moreover $J_{p-1} / J_{p}$ is dense and holomorphically closed in

$$
A_{p}:=C_{0}\left(X_{p}, X_{p-1} ; M_{N}(\mathbb{C})\right)^{\Gamma} .
$$

The density theorem for K-theory [Bost], Théorème A.2.1, tells us that the inclusion $J_{p-1} / J_{p} \rightarrow A_{p}$ induces an isomorphism

$$
K_{*}\left(J_{p-1} / J_{p}\right) \rightarrow K_{*}\left(A_{p}\right) .
$$

The same arguments apply to the center of $J_{p-1} / J_{p}$, so there are natural isomorphisms

$$
\begin{aligned}
K_{*}\left(Z\left(J_{p-1} / J_{p}\right)\right) \otimes_{\mathbb{Z}} \mathbb{C} & \rightarrow \operatorname{HP}_{*}\left(Z\left(J_{p-1} / J_{p}\right)\right), \\
K_{*}\left(Z\left(J_{p-1} / J_{p}\right)\right) & \rightarrow K_{*}\left(Z\left(A_{p}\right)\right) .
\end{aligned}
$$

The spectrum of the algebras in (1.27) and (1.28) is $Y_{p}^{\prime} \backslash Z_{p}^{\prime}$ where

$$
\begin{aligned}
Y_{p}^{\prime} & =\operatorname{Prim}\left(Z\left(A_{\text {smooth }} / J_{p}\right)\right)=Y_{p} \cap \theta_{p}^{-1}\left(X_{p}^{\prime} / \Gamma\right), \\
Z_{p}^{\prime} & =\left\{J \in Y_{p}^{\prime} \mid Z\left(J_{p-1} / J_{p}\right) \subset J\right\}=Z_{p} \cap \theta_{p}^{-1}\left(X_{p}^{\prime} / \Gamma\right),
\end{aligned}
$$

with $\theta_{p}$ as in (1.24). Since the cardinality of $\theta_{p}^{-1}(x)$ is locally constant for $x \in$ $X_{p} \backslash X_{p-1}$, any $\Gamma$-equivariant diffeotopy implementing the diffeotopy equivalence $X_{p}^{\prime} \rightarrow X_{p}$ naturally gives rise to a diffeotopy for the inclusion map $\left(Y_{p}^{\prime}, Z_{p}^{\prime}\right) \rightarrow$ $\left(Y_{p}, Z_{p}\right)$. Therefore

$$
\breve{H}^{n}\left(Y_{p}, Z_{p} ; \mathbb{C}\right) \rightarrow \check{H}^{n}\left(Y_{p}^{\prime}, Z_{p}^{\prime} ; \mathbb{C}\right)
$$

is an isomorphism for all $n$.

Furthermore $A_{p}$ is a finite direct sum of algebras of the form $C_{0}\left(Y, Z ; M_{k}(\mathbb{C})\right)$ with $Y$ a connected manifold. The center of such an algebra is $C_{0}(Y, Z)$, which clearly is Morita-equivalent to the algebra itself. Hence

$$
Z\left(A_{p}\right)=C_{0}\left(Y_{p}^{\prime}, Z_{p}^{\prime}\right)
$$


and the inclusion map induces an isomorphism

$$
K_{*}\left(C_{0}\left(Y_{p}^{\prime}, Z_{p}^{\prime}\right)\right) \rightarrow K_{*}\left(A_{p}\right) .
$$

Returning to the smooth level we note that

$$
Z\left(J_{p-1} / J_{p}\right):=\widetilde{C}_{0}^{\infty}\left(Y_{p}^{\prime}, Z_{p}^{\prime}\right)
$$

satisfies the conditions 1 and 2 of Theorem 1.6. The proof of Theorem 1.6 yields a natural isomorphism

$$
\mathrm{HP}_{*}\left(\widetilde{C}_{0}^{\infty}\left(Y_{p}^{\prime}, Z_{p}^{\prime}\right)\right) \cong H^{[*]}\left(Y_{p}^{\prime}, Z_{p}^{\prime}\right) .
$$

Combining all the above we obtain a diagram

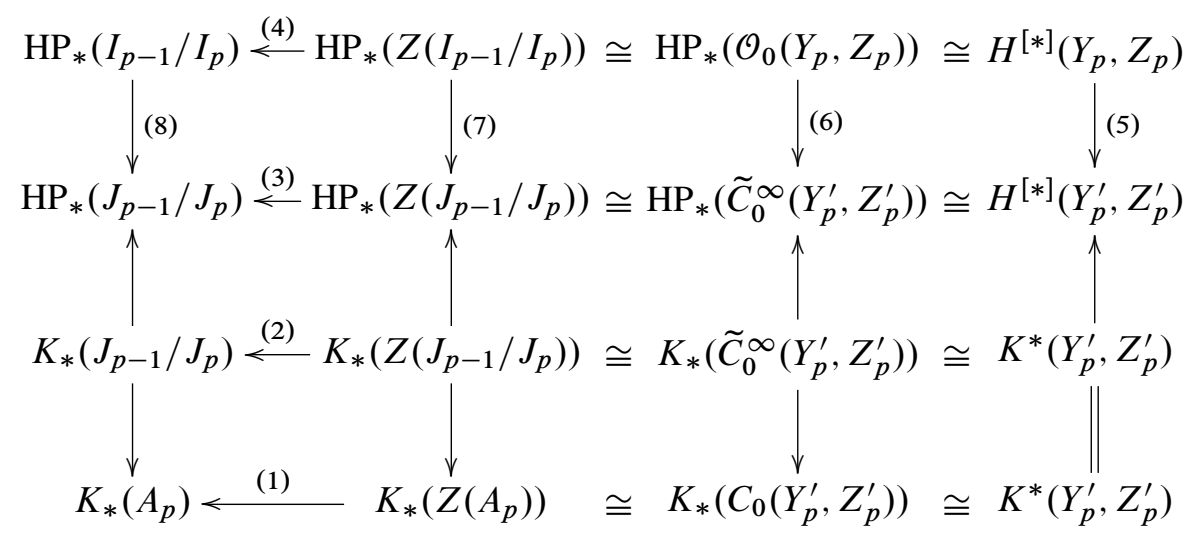

which is commutative because all the maps are natural. So far we know that

- the maps from row 3 to row 4 are isomorphisms by the density theorem in topological K-theory,

- the Chern characters from row 3 to row 2 become isomorphisms after tensoring with $\mathbb{C}$,

- (1), (4) and (5) are isomorphisms.

With some obvious diagram chases we first deduce that (2) and (3) are isomorphisms, and then that (6), (7) and finally (8) are isomorphisms.

Example. Let $X=\mathbb{C}^{2}, X^{\prime}=[-1,1]^{2} \subset \mathbb{R}^{2} \subset \mathbb{C}^{2}$ and $\Gamma=\{ \pm 1\}^{2}$. We describe the stratifications of the spectra of the algebras

$$
A_{\text {alg }}=\mathcal{O}(X) \rtimes \Gamma, \quad A_{\text {smooth }}=C^{\infty}\left(X^{\prime}\right) \rtimes \Gamma .
$$

First the strata of $X$ and $X^{\prime}$ :

$$
\begin{array}{ll}
X_{0}=\{(0,0)\}, & X_{0}^{\prime}=\{(0,0)\}, \\
X_{1}=\{0\} \times \mathbb{C} \cup \mathbb{C} \times\{0\}, & X_{1}^{\prime}=\{0\} \times[-1,1] \cup[-1,1] \times\{0\}, \\
X_{2}=\mathbb{C}^{2}, & X_{2}^{\prime}=[-1,1]^{2} .
\end{array}
$$


Let $\sigma$ and $\tau$ be the two irreducible representations of the group $\{ \pm 1\}$. We extend them to representations $\sigma_{0}, \tau_{0}$ of $C^{\infty}([-1,1]) \rtimes\{ \pm 1\}$ with central character $0 \in[-1,1]$. The centers of the filtration are

$$
\begin{aligned}
& Y_{0}=\left\{\sigma_{0}, \tau_{0}\right\}^{2}, \\
& Y_{1}=\left\{\sigma_{0}, \tau_{0}\right\} \times \mathbb{C} /\{ \pm 1\} \cup \mathbb{C} /\{ \pm 1\} \times\left\{\sigma_{0}, \tau_{0}\right\} / \sim \cong\{0\} \times \mathbb{C} \cup \mathbb{C} \times\{0\}, \\
& Y_{2}=X / \Gamma \cong(\mathbb{C} /\{ \pm 1\})^{2}, \\
& Y_{0}^{\prime}=\left\{\sigma_{0}, \tau_{0}\right\}^{2}, \\
& Y_{1}^{\prime}=\left\{\sigma_{0}, \tau_{0}\right\} \times[-1,1] \cup[-1,1] \times\left\{\sigma_{0}, \tau_{0}\right\} / \sim \cong\{0\} \times[-1,1] \cup[-1,1] \times\{0\}, \\
& Y_{2}^{\prime}=X^{\prime} / \Gamma \cong[0,1]^{2},
\end{aligned}
$$

where the equivalence relation $\sim$ identifies all the points lying over $(0,0) \in \mathbb{C}^{2}$. Next we write down the subcenters of the filtration:

$$
\begin{array}{ll}
Z_{0}=\emptyset, & Z_{0}^{\prime}=\emptyset, \\
Z_{1}=\{(0,0)\}, & Z_{1}^{\prime}=\{(0,0)\}, \\
Z_{2}=X_{1}, & Z_{2}^{\prime}=X_{1}^{\prime} .
\end{array}
$$

Finally we mention the primitive ideal spectra of the subquotients of the filtrations:

$$
\begin{array}{ll}
\left(Y_{0} \backslash Z_{0}\right) / \Gamma \cong 4 \text { points, } & \left(Y_{0}^{\prime} \backslash Z_{0}^{\prime}\right) / \Gamma \cong 4 \text { points, } \\
\left(Y_{1} \backslash Z_{1}\right) / \Gamma \cong\left\{\sigma_{0}, \tau_{0}\right\} \times \mathbb{C}^{\times} /\{ \pm 1\}, & \left(Y_{1}^{\prime} \backslash Z_{1}^{\prime}\right) / \Gamma \cong\left\{\sigma_{0}, \tau_{0}\right\} \times(0,1], \\
\left(Y_{2} \backslash Z_{2}\right) / \Gamma \cong\left(\mathbb{C}^{\times} /\{ \pm 1\}\right)^{2}, & \left(Y_{2}^{\prime} \backslash Z_{2}^{\prime}\right) / \Gamma \cong(0,1]^{2}
\end{array}
$$

\section{Some representation theory of reductive $p$-adic groups}

2.1. Convolution algebras. In this chapter we collect some important results concerning smooth representations of reductive $p$-adic groups. Good sources for the theory discussed here are [BeDe], [Car], [Sil], [SSZ], [Tits], [Wal].

Let $\mathbb{F}$ be a non-Archimedean local field with discrete valuation $v$ and norm $\|\cdot\|_{\mathbb{F}}$. We assume that the cardinality of the residue field is a power $q$ of a prime $p$. Let $\mathcal{E}$ be a connected reductive algebraic group defined over $\mathbb{F}$, and let $G=\mathscr{H}(\mathbb{F})$ be the group of $\mathbb{F}$-rational points. We briefly call $G$ a reductive $p$-adic group.

We denote the collection of compact open subgroups of $G$ by $\operatorname{CO}(G)$. A representation $V$ of $G$ is called smooth if every $v \in V$ is fixed by a compact open subgroup, or equivalently if $V=\bigcup_{K \in \operatorname{CO}(G)} V^{K}$. We say that such a smooth representation $V$ is admissible if every $V^{K}$ has finite dimension. For example every smooth $G$-representation of finite length is admissible [BeDe], 3.12. Let $\operatorname{Rep}(G)$ be the category of smooth $G$-representations on complex vector spaces, and let $\operatorname{Irr}(G)$ be the set of equivalence classes of irreducible objects in $\operatorname{Rep}(G)$. The Jordan-Hölder 
content $\mathrm{JH}(V)$ is the collection of all elements of $\operatorname{Irr}(G)$ which are equivalent to a subquotient of the $G$-representation $V$.

Fix a Haar measure $d \mu$ on $G$. Recall that the convolution product of two functions $f, f^{\prime}: G \rightarrow \mathbb{C}$ is defined as

$$
\left(f * f^{\prime}\right)\left(g^{\prime}\right)=\int_{G} f(g) f^{\prime}\left(g^{-1} g^{\prime}\right) d \mu(g) .
$$

For $K \in \mathrm{CO}(G)$ we let $\mathscr{H}(G, K)$ be the convolution algebra of $K$-biinvariant complex-valued compactly supported functions on $G$. This is called the Hecke algebra of $(G, K)$. Our main subject of study will be the Hecke algebra of $G$, which consists of all compactly supported locally constant functions on $G$ :

$$
\mathscr{H}(G):=\bigcup_{K \in \operatorname{CO}(G)} \mathcal{H}(G, K) .
$$

For every $K \in \operatorname{CO}(G)$ there is an idempotent $e_{K} \in \mathscr{H}(G)$, which is $\mu(K)^{-1}$ times the characteristic function of $K$. Notice that

$$
\mathscr{H}(G, K)=e_{K} \mathscr{H}(G) e_{K}=\mathscr{H}(G)^{K \times K},
$$

where $G \times G$ acts on $\mathscr{H}(G)$ by left and right translations. In particular, the nonunital algebra $\mathscr{H}(G)$ is idempotented, which assures that many properties of unital algebras also hold for $\mathscr{H}(G)$.

An $\mathscr{H}(G)$-module $V$ is called nondegenerate or essential if

$$
\mathscr{H}(G) \cdot V=V,
$$

or equivalently if for all $v \in V$ there exists a $K \in \operatorname{CO}(G)$ such that $e_{K} \cdot v=v$. A smooth $G$-representation $(\pi, V)$ is made into an essential $\mathscr{H}(G)$-module by

$$
\pi(f) v=\int_{G} f(g) \pi(g) v d \mu(g), \quad v \in V, f \in \mathscr{H}(G) .
$$

This leads to an equivalence between $\operatorname{Rep}(G)$ and the category of essential $\mathscr{H}(G)$ modules. Hence we may identify the primitive ideal spectrum of $\mathscr{H}(G)$ with $\operatorname{Irr}(G)$.

Let $S(G, K)$ be the space of rapidly decreasing $K$-biinvariant functions on $G$. According to [Vig], Theorem 29, this is a unital nuclear Fréchet *-algebra. HarishChandra's Schwartz algebra consists of all uniformly locally constant rapidly decreasing functions on $G$ :

$$
\mathcal{S}(G):=\bigcup_{K \in \operatorname{CO}(G)} \mathcal{S}(G, K) .
$$

Endowed with the inductive limit topology this is a complete locally convex topological algebra with separately continuous multiplication. Clearly

$$
\delta(G, K)=e_{K} \delta(G) e_{K}=S(G)^{K \times K} .
$$


If $\left(K_{i}\right)_{i=1}^{\infty}$ is a decreasing sequence of compact open subgroups of $G$ which forms a neighborhood basis of the unit element $e \in G$, then

$$
\delta(G)=\bigcup_{i=1}^{\infty} \delta\left(G, K_{i}\right)
$$

is a strict inductive limit of nuclear Fréchet spaces. Nevertheless $\delta(G)$ is not metrizable. We say that a smooth $G$-representation $(\pi, V)$ is tempered if the $\mathscr{H}(G)$-module structure extends to $\delta(G)$. If $(\pi, V)$ is admissible, then there is at most one such extension; see [SSZ], p. 51. Thus we have

- the category $\operatorname{Rep}^{t}(G)$ of tempered smooth $G$-representations,

- the space $\operatorname{Irr}^{t}(G)$ of equivalence classes of irreducible objects in $\operatorname{Rep}^{t}(G)$,

- the primitive ideal spectrum of $\delta(G)$, which by [SSZ], p. 52, can be identified with $\operatorname{Irr}^{t}(G)$.

Furthermore we consider the reduced $C^{*}$-algebra of $G$. By definition $C_{r}^{*}(G)$ is the completion of $\mathscr{H}(G)$ with respect to the operator norm coming from the left regular representation of $G$ on $L^{2}(G)$. For $K \in \operatorname{CO}(G)$ let $C_{r}^{*}(G, K)$ be the norm closure of $\mathscr{H}(G, K)$ in $B\left(L^{2}(G)\right)$. This is a unital type I $C^{*}$-algebra which contains $\delta(G, K)$ as a holomorphically closed dense subalgebra [Vig], Theorem 29. Moreover by [SSZ], p. 53,

$$
C_{r}^{*}(G, K)=e_{K} C_{r}^{*}(G) e_{K}=C_{r}^{*}(G)^{K \times K} .
$$

Therefore we can construct $C_{r}^{*}(G)$ also as an inductive limit of $C^{*}$-algebras:

$$
C_{r}^{*}(G)=\lim _{K \in \operatorname{CO}(G)} C_{r}^{*}(G, K) .
$$

Having introduced these algebras we will describe the Bernstein decomposition of $\operatorname{Rep}(G)$. Suppose that $P$ is a parabolic subgroup of $G$ and that $P=M \ltimes N$ where $N$ is the unipotent radical of $P$ and $M$ is a Levi subgroup. Although $G$ and $M$ are unimodular the modular function $\delta_{P}$ of $P$ is general not constant. To be precise,

$$
\delta_{P}(m n)=\left\|\operatorname{det}\left(\left.\operatorname{ad}(m)\right|_{\mathfrak{n}}\right)\right\|_{\mathbb{F}}, \quad m \in M, n \in N,
$$

where $\mathfrak{n}$ is the Lie algebra of $N$. For $\sigma \in \operatorname{Rep}(M)$ one defines

$$
I_{P}^{G}(\sigma):=\operatorname{Ind}_{P}^{G}\left(\delta_{P}^{1 / 2} \otimes \sigma\right) .
$$

This means that we first inflate $\sigma$ to $P$, then we twist it with $\delta_{P}^{1 / 2}$ and finally we take the smooth induction to $G$. The twist is useful to preserve unitarity. The functor $I_{P}^{G}$ is known as parabolic induction. It is transitive in the sense that for any parabolic subgroup $Q \subset P$ we have

$$
I_{Q}^{G}=I_{P}^{G} \circ I_{Q \cap M}^{M}
$$


Let $\sigma$ be an irreducible supercuspidal representation of $M$. Thus the restriction of $\sigma$ to the derived group of $M$ is unitary, but $Z(M)$ may act on $\sigma$ by an arbitrary character. We call $(M, \sigma)$ a cuspidal pair, and from it we construct the parabolically induced $G$-representation $I_{P}^{G}(\sigma)$. For every $(\pi, V) \in \operatorname{Irr}(G)$ there is a cuspidal pair $(M, \sigma)$, uniquely determined up to $G$-conjugacy, such that $V \in \operatorname{JH}\left(I_{P}^{G}(\sigma)\right)$.

We denote the complex torus of nonramified characters of $M$ by $X_{\mathrm{nr}}(M)$, and the compact subtorus of unitary nonramified characters by $X_{\text {unr }}(M)$. We say that two cuspidal pairs $(M, \sigma)$ and $\left.M^{\prime}, \sigma^{\prime}\right)$ are inertially equivalent if there exist $\chi \in X_{\mathrm{nr}}\left(M^{\prime}\right)$ and $g \in G$ such that $M^{\prime}=g M g^{-1}$ and $\sigma^{\prime} \otimes \chi \cong \sigma^{g}$. With an inertial equivalence class $\mathfrak{s}=[M, \sigma]_{G}$ we associate a subcategory $\operatorname{Rep}(G)^{\mathfrak{s}}$ of $\operatorname{Rep}(G)$. By definition its objects are smooth $G$-representations $\pi$ with the following property: for every $\rho \in \mathrm{JH}(\pi)$ there is a $(M, \sigma) \in \mathfrak{s}$ such that $\rho$ is a subrepresentation of $I_{P}^{G}(\sigma)$. These blocks $\operatorname{Rep}(G)^{\mathfrak{s}}$ give rise to the Bernstein decomposition [BeDe], Proposition 2.10,

$$
\operatorname{Rep}(G)=\prod_{\mathfrak{s} \in \Omega(G)} \operatorname{Rep}(G)^{\mathfrak{s}} .
$$

The set $\Omega(G)$ of Bernstein components is countably infinite. There are corresponding decompositions of the Hecke and Schwartz algebras of $G$ into two-sided ideals:

$$
\begin{aligned}
\mathscr{H}(G) & =\bigoplus_{\mathfrak{s} \in \Omega(G)} \mathcal{H}(G)^{\mathfrak{s}}, \\
\mathcal{S}(G) & =\bigoplus_{\mathfrak{s} \in \Omega(G)} \mathcal{S}(G)^{\mathfrak{s}} .
\end{aligned}
$$

For $C_{r}^{*}(G)$ this is a less straightforward since its elements can be supported on infinitely many Bernstein components. Let $C_{r}^{*}(G)^{\mathfrak{s}}$ be the two-sided ideal generated by $\mathscr{H}(G)^{\mathfrak{s}}$. The reduced $C^{*}$-algebra of $G$ decomposes as a direct sum in the $C^{*}$ algebra sense,

$$
C_{r}^{*}(G)=\underset{\mathfrak{S}}{\lim _{\mathfrak{s} \in \mathscr{S}}} \bigoplus_{r} C_{r}^{*}(G)^{\mathfrak{s}},
$$

where the direct limit runs over all finite subsets $\subseteq$ of $\Omega(G)$. For $K \in \operatorname{CO}(G)$ and $\mathfrak{s} \in \Omega(G)$ we write

$$
\begin{aligned}
\mathscr{H}(G, K)^{\mathfrak{s}} & =\mathscr{H}(G)^{\mathfrak{s}} \cap \mathscr{H}(G, K), \\
\mathcal{S}(G, K)^{\mathfrak{s}} & =\delta(G)^{\mathfrak{s}} \cap \mathcal{S}(G, K), \\
C_{r}^{*}(G, K)^{\mathfrak{s}} & =C_{r}^{*}(G)^{\mathfrak{s}} \cap C_{r}^{*}(G, K) .
\end{aligned}
$$

Every element of $\mathscr{H}(G)$ has a unique decomposition as a sum of a part in $\mathscr{H}(G)^{\mathfrak{s}}$ and a part in the annihilator of this ideal. In particular we can write

$$
e_{K}=e_{K}^{\mathfrak{F}}+e_{K}^{\prime} \in \mathscr{H}(G)^{\mathfrak{s}} \oplus \underset{\mathfrak{s}^{\prime} \in \Omega(G) \backslash\{\mathfrak{s}\}}{\bigoplus} \mathcal{H}(G)^{\mathfrak{s}^{\prime}} .
$$


Proposition 2.1. a) For fixed $K \in \mathrm{CO}(G)$ there exist only finitely many $s \in \Omega(G)$ such that $\mathscr{H}(G, K)^{\mathfrak{s}} \neq 0$.

b) For every $\mathfrak{s} \in \Omega(G)$ there exists a $K_{\mathfrak{s}} \in \mathrm{CO}(G)$ such that for all compact open subgroups $K \subset K_{\mathfrak{s}}$ the bimodules $e_{K}^{\mathfrak{S}} \mathscr{H}(G)$ and $\mathscr{H}(G) e_{K}^{\mathfrak{s}}$ provide a Morita equivalence between

$$
\mathscr{H}(G)^{\mathfrak{s}}=\mathscr{H}(G) e_{K}^{\mathfrak{S}} \mathscr{H}(G) \text { and } \mathscr{H}(G, K)^{\mathfrak{s}}=e_{K}^{\mathfrak{s}} \mathscr{H}(G) e_{K}^{\mathfrak{s}}
$$

c) As b) but with $S(G)$ instead of $\mathscr{H}(G)$.

d) As b) but with $C_{r}^{*}(G)$ instead of $\mathscr{H}(G)$.

Proof. a) See [BeDe], $\$ 3.7$.

b) By [BeDe], Corollaire 3.9, there exists a $K_{\mathfrak{s}} \in \mathrm{CO}(G)$ such that

$$
\mathscr{H}(G)^{\mathfrak{s}}=\mathscr{H}(G) e_{K_{\mathfrak{s}}^{\mathfrak{s}}} \mathscr{H}(G)
$$

For any $K \in \operatorname{CO}(G)$ with $K \subset K_{\mathfrak{s}}$ we have $e_{K_{\mathfrak{s}}} \in \mathscr{H}(G) e_{K} \mathscr{H}(G)$. Hence

$$
\mathscr{H}(G) e_{K}^{\mathfrak{F}} \mathscr{H}(G)=\left(\mathscr{H}(G) e_{K} \mathscr{H}(G)\right) \cap \mathscr{H}(G)^{\mathfrak{s}}
$$

contains $e_{K_{\mathfrak{S}}}^{\mathfrak{S}}$ and must equal $\mathscr{H}(G)^{\mathfrak{S}}$. We note that these $e_{K}^{\mathfrak{S}}$ are precisely the special idempotents constructed in [BuKu], Proposition 3.13.

c) and d) follow directly from b) and the characterisation of $S(G)^{\mathfrak{s}}$ and $C_{r}^{*}(G)^{\mathfrak{s}}$ as the ideals generated by $\mathscr{H}(G)^{\mathfrak{s}}$.

$$
\begin{aligned}
& \text { Let } \mathfrak{s}=[M, \sigma]_{G} \in \Omega(G) \text { and put } \\
& \qquad \begin{aligned}
N(M, \sigma) & =\left\{g \in N_{G}(M) \mid \sigma^{g} \cong \sigma \otimes \chi \text { for some } \chi \in X_{\mathrm{nr}}(M)\right\}, \\
W_{\mathfrak{s}} & =N(M, \sigma) / M .
\end{aligned}
\end{aligned}
$$

Theorem 2.2. For $K \in \mathrm{CO}(G)$ as in Proposition 2.1.b $\mathscr{H}(G, K)^{\mathfrak{s}}$ is a unital finite type algebra with center isomorphic to

$$
\mathcal{O}\left(X_{\mathrm{nr}}(M) / W_{\mathfrak{s}}\right)=\mathcal{O}\left(X_{\mathrm{nr}}(M)\right)^{W_{\mathfrak{s}}} .
$$

There are natural isomorphisms

$$
\begin{aligned}
Z\left(\mathcal{H}\left(G, K_{\mathfrak{\Im}}\right)^{\mathfrak{I}}\right) & \cong Z\left(\operatorname{Rep}(G)^{\mathfrak{F}}\right), \\
\operatorname{Prim}\left(\mathcal{H}\left(G, K_{\mathfrak{s}}\right)^{\mathfrak{I}}\right) & \cong \operatorname{Irr}(G) \cap \operatorname{Rep}(G)^{\mathfrak{s}}, \\
\operatorname{Prim}\left(S\left(G, K_{\mathfrak{s}}\right)^{\mathfrak{S}}\right) & \cong \operatorname{Irr}^{t}(G) \cap \operatorname{Rep}(G)^{\mathfrak{s}} .
\end{aligned}
$$

Proof. This follows from Théorème 2.13 and Corollaire 3.4 of [BeDe], in combination with Proposition 2.1. 
The cohomological dimension of the abelian category $\operatorname{Rep}(G)$ equals the rank of $G$, which is by definition the dimension of a maximal split subtorus of $G$ [ScSt], Section II.3. The author does not know whether the abelian category $\operatorname{Rep}^{t}(G)$ has finite cohomological dimension. Yet one can determine something like the global dimension of $\delta(G)$, at the cost of using more advanced techniques. Namely, according to Meyer [Mey2], Theorem 29, the cohomological dimension of the exact category $\operatorname{Mod}_{b}(S(G))$ of complete essential bornological $S(G)$-modules is also equal to the rank of $G$. The natural tensor product to work with in this category is the completed bornological $A$-balanced tensor product, which we denote by $\widehat{\otimes}_{A}$. For Fréchet spaces $\hat{\otimes}_{\mathbb{C}}$ agrees with the completed projective tensor product, so we abbreviate it to $\hat{\otimes}$. For later use we translate these cohomological dimensions to statements about Hochschild homology.

Lemma 2.3. a) $\mathrm{HH}_{n}(\mathscr{H}(G))=0$ for all $n>\operatorname{rk}(G)$,

b) $\mathrm{HH}_{n}\left(S(G), \widehat{\otimes}_{\mathbb{C}}\right)=0$ for all $n>\operatorname{rk}(G)$.

Proof. a) can be found in [Nis], but we prefer to derive it from the above. Let

$$
\mathbb{C} \leftarrow P_{0} \leftarrow P_{1} \leftarrow \cdots \leftarrow P_{\operatorname{rk}(G)} \leftarrow 0
$$

be a projective resolution of the trivial $G$-module $\mathbb{C}$. Endowing $P_{m} \otimes \mathscr{H}(G)$ with the diagonal $G$-action, $\mathscr{H}(G) \leftarrow P_{*} \otimes \mathscr{H}(G)$ becomes a resolution of $\mathscr{H}(G)$ by projective $\mathscr{H}(G)$-bimodules. By definition

$$
\begin{aligned}
& \mathrm{HH}_{n}(\mathscr{H}(G))=\operatorname{Tor}_{n}^{\mathscr{H}(G) \otimes \mathscr{H}(G)^{\mathrm{op}}}(\mathcal{H}(G), \mathscr{H}(G)) \\
& =H_{n}\left(\operatorname{Hom}_{\mathscr{H}(G) \otimes \mathscr{H}(G)^{\mathrm{op}}}\left(P_{*} \otimes \mathscr{H}(G), \mathcal{H}(G)\right)\right),
\end{aligned}
$$

which clearly vanishes for $n>\operatorname{rk}(G)$.

b) We will use that the inclusion $\mathscr{H}(G) \rightarrow S(G)$ is isocohomological [Mey2], Theorem 22. According to [Mey2], (22), the differential complex

$$
S(G) \leftarrow S(G) \hat{\otimes}_{\mathscr{H}(G)} P_{*} \hat{\otimes}_{\mathbb{C}} S(G)
$$

is a projective resolution of $S(G)$ in $\operatorname{Mod}_{b}(S(G))$. Hence

$$
\begin{aligned}
\mathrm{HH}_{n}\left(S(G), \hat{\otimes}_{\mathbb{C}}\right) & =\operatorname{Tor}_{n}^{S(G) \hat{\otimes} S(G)^{\mathrm{op}}}(S(G), S(G)) \\
& =H_{n}\left(\operatorname{Hom}_{\mathcal{S}(G) \hat{\otimes} S(G)^{\mathrm{op}}}\left(\mathcal{S}(G) \hat{\otimes}_{\mathcal{H}(G)} P_{*} \hat{\otimes}_{\mathbb{C}} \mathcal{S}(G), \mathcal{S}(G)\right)\right) .
\end{aligned}
$$

From (2.15) we see immediately that this vanishes for all $n>\operatorname{rk}(G)$.

Corollary 2.4. Let $\mathfrak{s} \in \Omega(G), n>\operatorname{rk}(G)$ and $K \in \operatorname{CO}(G)$ be such that $K \subset K_{\mathfrak{s}}$.

a) $\mathrm{HH}_{n}\left(\mathscr{H}(G)^{\mathfrak{s}}\right)=0=\mathrm{HH}_{n}\left(\mathscr{H}(G, K)^{\mathfrak{s}}\right)$.

b) $\mathrm{HH}_{n}\left(S(G)^{\mathfrak{s}}, \hat{\otimes}_{\mathbb{C}}\right)=0=\mathrm{HH}_{n}\left(S(G, K)^{\mathfrak{s}}, \hat{\otimes}\right)$. 
Proof. b) From (2.12) and (2.17) we see that

$$
\mathrm{HH}_{n}\left(S(G), \hat{\otimes}_{\mathbb{C}}\right) \cong \bigoplus_{\mathfrak{s} \in \Omega(G)} \mathrm{HH}_{n}\left(S(G)^{\mathfrak{s}}, \hat{\otimes}_{\mathbb{C}}\right)
$$

By Proposition 2.1.c,

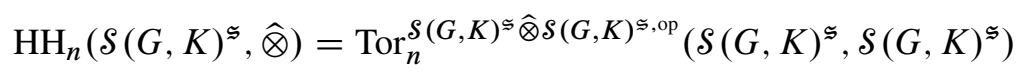

$$
\begin{aligned}
& \cong \operatorname{Tor}_{n}^{\mathcal{S}(G)^{\mathfrak{s}} \hat{\otimes} \delta(G)^{\mathfrak{s}, \text { op }}}\left(\mathcal{S}(G)^{\mathfrak{F}}, \mathcal{S}(G)^{\mathfrak{s}}\right) \\
& =\mathrm{HH}_{n}\left(S(G)^{\mathfrak{s}}, \hat{\otimes}_{\mathbb{C}}\right) \text {, }
\end{aligned}
$$

where we take the torsion functors in the category of complete bornological modules. By Lemma 2.3.b these homology groups all vanish for $n>\operatorname{rk}(G)$.

a) can be proved in exactly the same way as b), using (2.11), Lemma 2.3.a and Proposition 2.1.b.

2.2. The Plancherel theorem. The Plancherel formula for $G$ is an explicit decomposition of the trace

$$
\mathscr{H}(G) \rightarrow \mathbb{C}, \quad f \mapsto f(e)
$$

in terms of the traces of irreducible $G$-representations. Closely related is the Plancherel theorem, which describes $\delta(G)$ in terms of its irreducible representations. This description is due to Harish-Chandra [HC1], [HC2], although he published only a sketch of the proof. Harish-Chandra's notes were worked out in detail by Waldspurger [Wal]. In the present section we recall the most important ingredients of the Plancherel theorem, relying almost entirely on the above papers.

A parabolic pair $(P, A)$ consists of a parabolic subgroup $P$ of $G$ and a maximal split torus $A$ in the center of some Levi subgroup $M$ of $P$. If $N$ is the unipotent radical of $P$ then $P=M \ltimes N$ and $M=Z_{G}(A)$. Moreover restriction from $M$ to $A$ defines a surjection $X_{\mathrm{nr}}(M) \rightarrow X_{\mathrm{nr}}(A)$ with finite kernel.

The maximal parabolic pair is $\left(G, A_{G}\right)$, where $A_{G}$ is the unique maximal split torus of $Z(G)$. We fix a maximal split torus $A_{0}$ of $G$, and a minimal parabolic subgroup $P_{0}$ containing $A_{0}$. We call $(P, A)$ semi-standard if $A \subset A_{0}$, and standard if moreover $P \supset P_{0}$. Every parabolic pair is conjugate to a standard one.

Let $(\omega, E)$ be an irreducible square-integrable representation of $M$. By definition this entails that $E$ is smooth, pre-unitary and admissible. Let $(\breve{\omega}, \breve{E})$ be the smooth contragredient representation. The admissible $G \times G$-representation

$$
L(\omega, P)=I_{P \times P}^{G \times G}(E \otimes \breve{E})=I_{P}^{G}(E) \otimes I_{P}^{G}(\breve{E})
$$

is naturally a nonunital Hilbert algebra. Notice that for every $\chi \in X_{\text {unr }}(M)$ the representation $\omega \otimes \chi$ is also square-integrable, and that $L(\omega \otimes \chi, P)$ can be identified with $L(\omega, P)$. Let $\mathbf{k}_{\omega}$ be the set of $k \in X_{\mathrm{nr}}(M)$ such that $\omega \otimes k$ is equivalent to $\omega$. 
This is a finite subgroup of $X_{\text {unr }}(M)$. For every $k \in \mathbf{k}_{\omega}$ there exists a canonical unitary intertwiner

$$
I(k, \omega) \in \operatorname{Hom}_{G \times G}(L(\omega, P), L(\omega \otimes k, P)) .
$$

Next we consider the intertwiners associated to elements of various Weyl groups. Let $(Q, B)$ be another parabolic pair. Write $W(A|G| B)$ for the set of all homomorphisms $B \rightarrow A$ induced by inner automorphisms of $G$. This is a group in case $A=B$ :

$$
W(G, A):=W(A|G| A)=N_{G}(A) / Z_{G}(A)=N_{G}(A) / M .
$$

Let $\left(Q, A^{g}\right)$, with $g \in G$, be yet another parabolic pair, and put $n=[g] \in$ $W\left(A^{g}|G| A\right)$. The equivalence class of the $M^{g}$-representation $\left(\omega^{g^{-1}}, E\right)$ depends only on $n$ and may therefore be denoted by $n \omega$. Waldspurger constructs certain normalized intertwiners ${ }^{\circ} C_{Q \mid P}(n, \omega)$. Preferring the simpler notation $I(n, \omega)$ we recall their properties.

Theorem 2.5 ([Wal], Paragraphe V). Let $(P, A),\left(P^{\prime}, A^{\prime}\right)$ and $(Q, B)$ be semistandard parabolic pairs and let $n \in W(B|G| A)$. There exists an intertwiner

$$
I(n, \omega \otimes \chi) \in \operatorname{Hom}_{G \times G}(L(\omega, P), L(n \omega, Q))
$$

with the following properties:

- $\chi \rightarrow I(n, \omega \otimes \chi)$ is a rational function on $X_{\mathrm{nr}}(M)$,

- $I(n, \omega \otimes \chi)$ is unitary and regular for $\chi \in X_{\mathrm{unr}}(M)$,

- If $n^{\prime} \in W\left(A^{\prime}|G| B\right)$ then $I\left(n^{\prime}, n(\omega \otimes \chi)\right) I(n, \omega \otimes \chi)=I\left(n^{\prime} n, \omega \otimes \chi\right)$.

To define the Fourier transform implementing the Plancherel isomorphism we introduce a space of induction data such that every irreducible tempered representation is a direct summand of (at least) one of these parabolically induced representations. For every semi-standard parabolic pair $(P, A)$ choose a set $\Delta_{M}$ of irreducible squareintegrable representations of $M=Z_{G}(A)$, with the following property. For every square-integrable $\pi \in \operatorname{Irr}(M)$ there exists precisely one $\omega \in \Delta_{M}$ such that $\pi$ is equivalent to $\omega \otimes \chi$, for some $\chi \in X_{\mathrm{nr}}(M)$. We call a triple $(P, A, \omega)$ standard if $(P, A)$ is a standard parabolic pair and $\omega \in \Delta_{M}$.

An induction datum is a quadruple $(P, A, \omega, \chi)$ where $(P, A)$ is a semi-standard parabolic pair, $\omega \in \Delta_{M}$ and $\chi \in X_{\mathrm{nr}}(M)$. Let $\Xi$ be the scheme of all induction data and $\Xi_{u}$ the smooth submanifold of unitary induction data, that is, those with $\chi \in X_{\text {unr }}(M)$. Then $\Xi$ and $\Xi_{u}$ are countable disjoint unions of complex algebraic tori and compact tori, respectively. For $\xi=(P, A, \omega, \chi) \in \Xi$ we put

$$
I(\xi)=I_{P}^{G}(\omega \otimes \chi) .
$$


By [Wal], Lemme III.2.3, the representation $I(\xi)$ is tempered if and only if $\omega \otimes \chi$ is tempered, if and only if $\xi \in \Xi_{u}$. Like for cuspidal pairs one can define inertial equivalence on $\Xi_{u}$. The set $\Omega^{t}(G)$ of all equivalence classes $[P, A, \omega]_{G}$ is called the Harish-Chandra spectrum of $G$. It comes with a natural surjection $\Omega^{t}(G) \rightarrow \Omega(G)$, see [SSZ], Section 1. It follows from Proposition 2.1 and [Wal], Théorème VIII.1.2, that this map is finite-to-one.

Let $\mathscr{L}_{\Xi}$ be the vector bundle over $\Xi$ which is trivial on every component and whose fiber at $\xi$ is $L(\omega, P)$. We say that a section of this bundle is algebraic (polynomial) or rational if it is supported on only finitely many components, and has the required property on every component. Now we can define the Fourier transform:

$$
\mathscr{F}: \mathscr{H}(G) \rightarrow \mathcal{O}\left(\Xi ; \mathscr{L}_{\Xi}\right), \quad \mathcal{F}(f)(P, A, \omega, \chi)=I(P, A, \omega, \chi)(f) \in L(\omega, P),
$$

where the notation from (2.4) is used. Notice that this differs slightly from $\check{f}(\omega \otimes \chi, P)$ as in [Wal], $\S$ VII.1. To make it fit better with its natural adjoint Waldspurger adjusts the Fourier transform. We use (2.20) because it is multiplicative.

To formalize the action of the intertwiners on sections of $\mathscr{L}_{\Xi}$ we construct a locally finite groupoid $\mathcal{W}$. The set of objects of $\mathcal{W}$ is $\Xi$ and the morphisms from $\xi$ to $\xi^{\prime}$ are the pairs $(k, n)$ with the following properties:

- $k \in \mathbf{k}_{\omega}$,

- $n \in W\left(A|G| A^{\prime}\right)$ and $n\left(A^{\prime}\right)=A$,

- $n \omega^{\prime}$ is equivalent to $\omega \otimes \tilde{\chi}$ for some $\tilde{\chi} \in X_{\mathrm{nr}}(M)$.

The multiplication in $\mathcal{W}$, if possible, is

$$
(k, n)\left(k^{\prime}, n^{\prime}\right)=\left(k\left(k^{\prime} \circ n\right), n n^{\prime}\right) .
$$

Let $\Gamma\left(\Xi ; \mathscr{L}_{\Xi}\right)$ be a suitable algebra of sections of $\mathscr{L}_{\Xi}$. For $f \in \Gamma\left(\Xi ; \mathscr{L}_{\Xi}\right)$ we define

$$
k \cdot f(\chi)=I(k, \omega) f\left(k^{-1} \chi\right), \quad n \cdot f\left(\chi^{\prime}\right)=I(n, \omega) f\left(\chi^{\prime} \circ n\right) .
$$

Notice that the intertwiners do not stabilize $\mathcal{O}\left(\Xi ; \mathscr{L}_{\Xi}\right)$ in general. Nevertheless, we write $\mathcal{O}\left(\Xi ; \mathscr{L}_{\Xi}\right)^{W}$ for the subalgebra of $\mathcal{W}$-invariant sections, which by construction contains $\mathcal{F}(\mathscr{H}(G))$. Because $(\omega, E)$ is admissible,

$$
C^{\infty}\left(X_{\mathrm{unr}}(M)\right) \otimes L(\omega, P)^{K \times K}=C^{\infty}\left(X_{\mathrm{unr}}(M)\right) \otimes I_{P}^{G}(E)^{K} \otimes I_{P}^{G}(\breve{E})^{K}
$$

has a natural Fréchet topology for every $K \in \mathrm{CO}(G)$. We endow

$$
C_{c}^{\infty}\left(\Xi_{u} ; \mathscr{L}_{\Xi}\right)=\lim _{K \in \operatorname{CO}(G)} C_{c}^{\infty}\left(\Xi_{u} ; \mathscr{L}_{\Xi}^{K \times K}\right)
$$

with the inductive limit topology. The Plancherel theorem for reductive $p$-adic groups reads: 
Theorem 2.6 ([HC2], [Wal]). The Fourier transform

$$
\mathcal{F}: S(G) \rightarrow C_{c}^{\infty}\left(\Xi_{u} ; \mathscr{L}_{\Xi}\right)^{\mathcal{W}}
$$

is an isomorphism of topological algebras.

A simple representation theoretic consequence of this important theorem is

Corollary 2.7. For any $w \in \mathcal{W}$ and $\xi \in \Xi$ such that $w \xi$ is defined, the $G$-representations $I(\xi)$ and $I(w \xi)$ have the same irreducible subquotients, counted with multiplicity.

Proof. By [Cas], Corollary 2.3.3, we have to show that the traces of $I(\xi)$ and $I(w \xi)$ are the same, in other words, that the function

$$
\begin{aligned}
\mathscr{H}(G) \times X_{\mathrm{nr}}(M) & \rightarrow \mathbb{C}, \\
(f, \chi) & \mapsto \operatorname{tr} I(P, A, \omega, \chi)(f)-\operatorname{tr} I\left(w P, w A, w \omega, \chi \circ w^{-1}\right)(f),
\end{aligned}
$$

is identically 0 . Because this is a polynomial function of $\chi$, it suffices to show that it is 0 on $\mathscr{H}(G) \times X_{\text {unr }}(M)$. That follows from Theorem 2.6.

The Plancherel theorem can be used to describe the Fourier transform of $C_{r}^{*}(G)$. For $(\omega, E) \in \Delta_{M}$ let $\mathcal{K}(\omega, P)$ be the algebra of compact operators on the Hilbert space completion of $I_{P}^{G}(E)$. Notice that

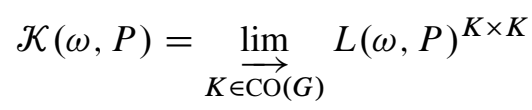

in the $C^{*}$-algebra sense, and that the intertwiner $I(n, \omega)$ extends to $\mathcal{K}(\omega, P)$ because it is unitary. Let $\mathcal{K}_{\Xi}$ be the vector bundle over $\Xi$ whose fiber at $(P, A, \omega, \chi)$ is $\mathcal{K}(\omega, P)$, and let $C_{0}\left(\Xi_{u} ; \mathcal{K}_{\Xi}\right)$ be the $C^{*}$-completion of

$$
\bigoplus_{(P, A, \omega)} C\left(X_{\mathrm{unr}}(M) ; \mathcal{K}(\omega, P)\right) .
$$

Theorem 2.8 ([Ply], Theorem 2.5). The Fourier transform extends to an isomorphism of $C^{*}$-algebras

$$
C_{r}^{*}(G) \rightarrow C_{0}\left(\Xi_{u} ; \mathcal{K}_{\Xi}\right)^{\mathcal{W}}
$$

We can also describe the images of the subalgebras $\delta(G, K)$ and $C_{r}^{*}(G, K)$ under the Fourier transform.

Theorem 2.9. Fix $K \in \mathrm{CO}(G)$. There exists a finite set of standard triples $\left(P_{i}, A_{i}, \omega_{i}\right)$ with the following properties. 
a) The Fourier transform yields algebra homomorphisms

$$
\begin{aligned}
\mathscr{H}(G, K) & \rightarrow \bigoplus_{i=1}^{n_{K}}\left(\mathcal{O}\left(X_{\mathrm{nr}}\left(M_{i}\right)\right) \otimes L\left(\omega_{i}, P_{i}\right)^{K \times K}\right)^{w_{i},} \\
\mathcal{S}(G, K) & \rightarrow \bigoplus_{i=1}^{n_{K}}\left(C^{\infty}\left(X_{\mathrm{unr}}\left(M_{i}\right)\right) \otimes L\left(\omega_{i}, P_{i}\right)^{K \times K}\right)^{w_{i}}, \\
C_{r}^{*}(G, K) & \rightarrow \bigoplus_{i=1}^{n_{K}}\left(C\left(X_{\mathrm{unr}}\left(M_{i}\right)\right) \otimes L\left(\omega_{i}, P_{i}\right)^{K \times K}\right)^{w_{i}},
\end{aligned}
$$

where $W_{i}$ is the isotropy group of $\left(P_{i}, A_{i}, \omega_{i}\right)$ in $W$.

b) The first map is injective, the second is an isomorphism of Fréchet algebras and the third is an isomorphism of $C^{*}$-algebras.

c) For every $w \in W_{i}$ there exists a unitary section

$$
u_{w} \in C^{\infty}\left(X_{\mathrm{unr}}\left(M_{i}\right)\right) \otimes L\left(\omega_{i}, P_{i}\right)^{K \times K}
$$

which extends to a rational section on $X_{\mathrm{nr}}\left(M_{i}\right)$ such that

$$
w f(\chi)=u_{w}(\chi) f\left(w^{-1} \chi\right) u_{w}^{-1}(\chi)
$$

for all $f \in C\left(X_{\mathrm{unr}}\left(M_{i}\right)\right) \otimes L\left(\omega_{i}, P_{i}\right)^{K \times K}$.

Proof. This is already proved in [Sol1], Theorem 10, but we include the proof anyway. Notice that, in contrast with Proposition 2.1, it is not necessary to require that $K$ is "small", except for being compact. That is because the most tricky (namely, not completely reducible) representations in a Bernstein component $[M, \sigma]_{G}$ appear only if we twist $\sigma$ by a nonunitary character $\chi \in X_{\mathrm{nr}}(M) \backslash X_{\mathrm{unr}}(M)$.

According to [Wal], Théorème VIII.1.2, there are only finitely many components in the Harish-Chandra spectrum $\Omega^{t}(G)$ on which the idempotent $e_{K}$ does not act as 0 . Pick one triple $\left(P_{i}, A_{i}, \omega_{i}\right)$ for each such component. Now a) and b) follow immediately from Theorems 2.6 and 2.8 .

Concerning c), every automorphism of

$$
L\left(\omega_{i}, P_{i}\right)^{K \times K} \cong \operatorname{End}_{\mathbb{C}}\left(I_{P}^{G}(E)^{K}\right)
$$

is inner, so (2.22) holds for some section $u_{w}$. Using Theorem 2.5 we can arrange that $u_{w}$ is rational on $X_{\mathrm{nr}}\left(M_{i}\right)$ and unitary on $X_{\mathrm{unr}}\left(M_{i}\right)$.

2.3. The Langlands classification. The Langlands classification describes the relation between the smooth spectrum of $G$ and the tempered spectra of its Levi subgroups. Let $(P, A)$ be a semi-standard parabolic pair, let $X^{*}(A)$ be the lattice of algebraic characters of $A$ and put

$$
a^{*}=X^{*}(A) \otimes_{\mathbb{Z}} \mathbb{R} \cong X^{*}(M) \otimes_{\mathbb{Z}} \mathbb{R} .
$$


For $A=A_{0}$ and $A=A_{G}$ we write $\mathrm{a}^{*}=\mathrm{a}_{0}^{*}$ and $\mathrm{a}^{*}=\mathrm{a}_{G}^{*}$, respectively. There is a natural homomorphism

$$
H_{M}: M \rightarrow \operatorname{Hom}_{\mathbb{Z}}\left(X^{*}(M), \mathbb{R}\right) \cong \operatorname{Hom}_{\mathbb{R}}\left(\mathfrak{a}^{*}, \mathbb{R}\right), q^{\left\langle\chi, H_{M}(m)\right\rangle}=\|\chi(m)\|_{\mathbb{F}},
$$

where $m \in M, \chi \in X^{*}(M)$ and $q$ is the cardinality of the residue field of $\mathbb{F}$. Conversely, for $v \in a^{*}$ we define a nonramified character $\chi_{\nu}$ of $M$ by

$$
\chi_{v}(m)=q^{\left\langle v, H_{M}(m)\right\rangle} .
$$

This yields an isomorphism

$$
a^{*} \cong \operatorname{Hom}\left(M, \mathbb{R}_{>0}\right)
$$

where Hom is taken in the category of topological groups. Let $Q$ be a parabolic subgroup such that $P \subset Q \subset G$, and let $q$ be its Lie algebra. It decomposes into $A$-eigenspaces

$$
\mathfrak{q}_{\alpha}:=\{x \in \mathfrak{q} \mid \operatorname{Ad}(a) x=\alpha(a) x \text { for all } a \in A\}
$$

with $\alpha \in X^{*}(A)$. The roots of $Q$ with respect to $A$ are

$$
\Sigma(Q, A):=\left\{\alpha \in X^{*}(A) \backslash\{1\} \mid \mathfrak{q}_{\alpha} \neq 0\right\} .
$$

The inclusions $A \rightarrow A_{0}$ and $M_{0} \rightarrow M$ identify $\mathrm{a}^{*}$ as a direct summand of $\mathrm{a}_{0}^{*}$. We have a root system

$$
\Sigma_{0}=\Sigma\left(G, A_{0}\right) \subset \mathrm{a}_{0}^{*}
$$

with positive roots $\Sigma\left(P_{0}, A_{0}\right)$, simple roots $\Delta_{0}=\Delta\left(P_{0}, A_{0}\right)$ and Weyl group $W_{0}=$ $W\left(P_{0}, A_{0}\right)$. We fix a $W_{0}$-invariant inner product $\langle,\rangle_{0}$ on $a_{0}^{*}$, so that we may identify this vector space with its dual. Denote the Lie algebras of $A$ and $A_{0}$ by a and $a_{0}$. (Notice that $a_{0}$ is not the dual of $a_{0}^{*}$, these are vector spaces over different fields.) For $F \subset \Delta_{0}$ we put

$$
\begin{aligned}
\Sigma_{F} & =\Sigma_{0} \cap \mathbb{R} F \subset \mathfrak{a}_{0}^{*}, & & W_{F}=\left\langle s_{\alpha} \mid \alpha \in F\right\rangle \subset W_{0}, \\
\mathfrak{a}_{F} & =\left\{x \in \mathfrak{a}_{0} \mid \alpha(x)=0 \text { for all } \alpha \in F\right\}, & & A_{F}=\exp \left(\mathfrak{a}_{F}\right), \\
\mathfrak{m}_{F} & =\left(\bigoplus_{\alpha \in \Sigma_{F}} \mathfrak{g}_{\alpha}\right) \oplus \mathfrak{a}_{0}, & & M_{F}=Z_{G}\left(A_{F}\right), \\
\mathfrak{n}_{F} & =\bigoplus_{\alpha \in \Sigma\left(P_{0}, A_{0}\right) \backslash \Sigma_{F}} \mathfrak{g}_{\alpha}, & & N_{F}=\exp \left(\mathfrak{n}_{F}\right), \\
\mathfrak{p}_{F} & =\left(\bigoplus_{\alpha \in \Sigma\left(P_{0}, A_{0}\right) \cup \Sigma_{F}} \mathfrak{g}_{\alpha}\right) \oplus \mathfrak{a}_{0}, & & P_{F}=M_{F} \ltimes N_{F} .
\end{aligned}
$$

Every standard parabolic pair is of the form $\left(P_{F}, A_{F}\right)$ for some $F \subset \Delta_{0}$. In this situation $F=\Delta\left(P_{F}, A_{F}\right)$ is the set of nonzero projections of $\Delta_{0} \subset \mathfrak{a}_{0}^{*}$ on $a_{F}^{*}$, and $W\left(M_{F}, A_{0}\right)=W_{F}$. In particular a standard parabolic pair is completely determined by either of its two ingredients. 
Furthermore we introduce the open and closed positive cones in $a^{*}$ :

$$
\begin{aligned}
& a^{*,+}=\left\{v \in a^{*} \mid\langle v, \alpha\rangle_{0}>0 \text { for all } \alpha \in \Delta(P, A)\right\}, \\
& \bar{a}^{*,+}=\left\{v \in a^{*} \mid\langle v, \alpha\rangle_{0} \geq 0 \text { for all } \alpha \in \Delta(P, A)\right\} .
\end{aligned}
$$

The antidual of $\bar{a}_{0}^{*,+}$ is the obtuse negative cone in $a_{0}^{*}$ :

$$
\bar{a}_{0}^{*,-}:=\left\{\mu \in a_{0}^{*} \mid\langle v, \mu\rangle \leq 0 \text { for all } v \in \bar{a}_{0}^{*,+}\right\} .
$$

The set of Langlands data $\Lambda^{+}$consists of all quadruples $\lambda=(P, A, \sigma, v)$ such that

- $(P, A)$ is a standard parabolic pair with Levi component $M=Z_{G}(A)$,

- $\sigma \in \operatorname{Irr}^{t}(M)$,

- $v \in a^{*,+}$.

Given a Langlands datum $\lambda \in \Lambda^{+}$we pick a concrete realization of $\sigma$ and we construct the admissible $G$-representation $I(\lambda)=I_{P}^{G}\left(\sigma \otimes \chi_{\nu}\right)$. Notice that $\lambda$ determines $I(\lambda)$ only modulo equivalence of $G$-representations. The classical Langlands classification for reductive $p$-adic groups reads:

Theorem 2.10. a) For every $\lambda \in \Lambda^{+}$the $G$-representation $I(\lambda)$ is indecomposable and has a unique irreducible quotient. We call this the Langlands quotient $J(\lambda)$.

b) For every $\pi \in \operatorname{Irr}(G)$ there is a unique $\lambda \in \Lambda^{+}$such that $\pi$ is equivalent to $J(\lambda)$.

Proof. See [Kon] or [BoWa], §XI.2. We note that Konno proves the uniqueness part only modulo $W_{0}$-conjugacy. However, two $W_{0}$-conjugate Langlands data are necessarily equal, which we will show in a more general setting in Lemma 2.13.

The Langlands datum of $\pi \in \operatorname{Irr}^{t}(G)$ is simply $\left(G, A_{G}, \pi, 0\right)$, and conversely $J(\lambda)$ cannot be tempered if $v \neq 0$. For every $\lambda \in \Lambda^{+}$the Langlands quotient is maximal among the irreducible constituents of $I(\lambda)$, in a suitable sense:

Lemma 2.11. Let $\lambda=(P, A, \sigma, v)$ and $\lambda^{\prime}=\left(P^{\prime}, A^{\prime}, \sigma^{\prime}, v^{\prime}\right)$ be Langlands data, and suppose that $J\left(\lambda^{\prime}\right) \in \mathrm{JH}(I(\lambda))$.

a) $v^{\prime}-v \in \bar{a}_{0}^{*,-}, A^{\prime} \subset A$ and $P^{\prime} \supset P$.

b) If $v^{\prime}=v$ then $\lambda^{\prime}=\lambda$.

c) $\operatorname{End}_{G}(I(\lambda))=\mathbb{C}$.

Proof. a) and b) The statements about $v$ and $v^{\prime}$ are [BoWa], Lemma XI.2.13. From the definition of $\Lambda^{+}$we see that $A^{\prime} \subset A$ and $P^{\prime} \supset P$ whenever $v^{\prime}-v \in \bar{a}_{0}^{*,-}$.

c) Let $\phi \in \operatorname{End}_{G}(I(\lambda))$ and write $M(\lambda)=\operatorname{ker}(I(\lambda) \rightarrow J(\lambda))$. By Theorem 2.10 and the above, $M(\lambda)$ and $J(\lambda)$ do not have any common irreducible constituents. Hence the composition

$$
M(\lambda) \stackrel{\phi}{\rightarrow} I(\lambda) \rightarrow J(\lambda)
$$


of $\phi$ with the quotient map is zero, and $\phi(M(\lambda)) \subset M(\lambda)$. Therefore $\phi$ induces a map $\phi_{J} \in \operatorname{End}_{G}(J(\lambda))$ and, because $J(\lambda)$ is irreducible, $\phi_{J}=\mu \mathrm{id}_{J(\lambda)}$ for some $\mu \in \mathbb{C}$. We want to show that

$$
\psi:=\phi-\mu \operatorname{id}_{I(\lambda)} \in \operatorname{End}_{G}(I(\lambda))
$$

equals zero. By construction $\psi(I(\lambda)) \subset M(\lambda)$. Let $V$ be an irreducible quotient representation of $M(\lambda)$ and consider the induced map $\psi_{V} \in \operatorname{Hom}_{G}(I(\lambda), V)$. It follows from Theorem 2.10.a that the smooth contragredient representation $I(\bar{\lambda})$ of $I(\lambda)$ has exactly one irreducible submodule, which moreover is equivalent to $J(\lambda)$. Since $\check{V}$ is not equivalent to the contragredient of $J(\lambda)$, we find

$$
0=\operatorname{Hom}_{G}(\check{V}, \check{I(\lambda)}) \cong \operatorname{Hom}_{G}(I(\lambda), V) .
$$

In particular $\psi_{V}=0$ and $\psi(I(\lambda)) \subset \operatorname{ker}(M(\lambda) \rightarrow V)$. Since $M(\lambda)$ has finite length [BeDe], p. 30, we conclude by induction that

$$
\operatorname{Hom}_{G}(I(\lambda), M(\lambda))=0 .
$$

Thus $\psi=0$ and $\phi=\mu \operatorname{id}_{I(\lambda)}$.

We would like to reformulate Lemma 2.11.b with a condition on $\sigma$ instead of $v$. To achieve this we will define a variation on the central character of a representation. Suppose that $\pi \in \operatorname{Irr}(G)$ belongs to the Bernstein component $\mathfrak{s}=[M, \rho]_{G}$. We may assume that $M$ is a standard Levi subgroup and that $\rho$ is a unitary supercuspidal $M$-representation. Pick $\chi_{\pi} \in X_{\mathrm{nr}}(M)$ such that $\pi \in \mathrm{JH}\left(I_{P}^{G}\left(\rho \otimes \chi_{\pi}\right)\right)$, and consider $\log \left|\chi_{\pi}\right| \in a^{*}$. This does not depend on the choice of $\rho$, and by Theorem 2.2 it is unique modulo $W_{\mathfrak{s}}$. However, we could have chosen another standard Levi subgroup $M^{\prime}$ conjugate to $M$. Since $W\left(A^{\prime}|G| A\right) \subset W_{0}$, this would lead to

$$
\log \left|\chi_{\pi}^{\prime}\right|=w \log \left|\chi_{\pi}\right| \in a_{0}^{*} \quad \text { for some } w \in W_{0} .
$$

Thus we get an invariant

$$
\operatorname{cc}_{G}(\pi):=W_{0} \log \left|\chi_{\pi}\right| \in \mathrm{a}_{0}^{*} / W_{0},
$$

which can be considered as a substitute for the absolute value of the $Z(\mathscr{H}(G))$ character of $\pi$. We note that, because the inner product on $a_{0}^{*}$ is $W_{0}$-invariant, $\left\|\operatorname{cc}_{G}(\pi)\right\|$ is well defined. Furthermore the orthogonal projection of $\operatorname{cc}_{G}(\pi)$ on $a_{G}^{*}$ consists of a single element, known as the central exponent of $\pi$. If $\pi \in \operatorname{JH}\left(I_{P_{F}}^{G}(\tau)\right)$ for some $\tau \in \operatorname{Irr}\left(M_{F}\right)$ then, by the transitivity of parabolic induction

$$
\operatorname{cc}_{G}(\pi)=W_{0} \operatorname{cc}_{M_{F}}(\tau) .
$$

Lemma 2.12. Let $\lambda=(P, A, \sigma, v)$ and $\lambda^{\prime}=\left(P^{\prime}, A^{\prime}, \sigma^{\prime}, v^{\prime}\right)$ be different Langlands data, and suppose that $J\left(\lambda^{\prime}\right) \in \mathrm{JH}(I(\lambda))$. Then

$$
\left\|\operatorname{cc}_{M^{\prime}}\left(\sigma^{\prime}\right)\right\|>\left\|\operatorname{cc}_{M}(\sigma)\right\| .
$$


Proof. By (2.29) all irreducible constituents of $I(\lambda)$ have the same $\operatorname{cc}_{G}$-invariant, so $\operatorname{cc}_{G}\left(J\left(\lambda^{\prime}\right)\right)=\operatorname{cc}_{G}(J(\lambda))$. We have

$$
\operatorname{cc}_{M}\left(\sigma \otimes \chi_{v}\right)=\operatorname{cc}_{M}(\sigma)+v=W_{M} \log \left|\chi_{\sigma}\right|+v \in a_{0}^{*} / W_{M} .
$$

Since $\sigma$ is irreducible and tempered, it is a unitary $M$-representation [Wal], Proposition III.4.1, in particular $\log \left|\chi_{\sigma}\right|=0$ on $A$. But $v \in \mathfrak{a}^{*}$ is zero on the derived group of $M$, so $\left\langle\log \left|\chi_{\sigma}\right|, v\right\rangle_{0}=0$. Hence

$$
\left\|\operatorname{cc}_{G}(J(\lambda))\right\|^{2}=\left\|\operatorname{cc}_{M}(\sigma)\right\|^{2}+\|v\|^{2},
$$

and similarly

$$
\left\|\operatorname{cc}_{G}\left(J\left(\lambda^{\prime}\right)\right)\right\|^{2}=\left\|\operatorname{cc}_{M^{\prime}\left(\sigma^{\prime}\right)}\right\|^{2}+\left\|v^{\prime}\right\|^{2} .
$$

By Lemma 2.9, $v^{\prime}-v \in \bar{a}_{0}^{*,-} \backslash\{0\}$, which by [Kon], Claim 3.5.1, implies that $\|v\|>\left\|v^{\prime}\right\|$. Since we have $\left\|\operatorname{cc}_{G}(J(\lambda))\right\|^{2}=\left\|\operatorname{cc}_{G}\left(J\left(\lambda^{\prime}\right)\right)\right\|^{2}$, it follows that $\left\|\operatorname{cc}_{M}(\sigma)\right\|^{2}<\left\|\operatorname{cc}_{M^{\prime}}\left(\sigma^{\prime}\right)\right\|^{2}$.

2.4. Parametrizing irreducible representations. We will combine the Langlands classification with the Plancherel theorem to parametrize the irreducible smooth $G$ representations. This parametrization is not complete, in the sense that certain packets contain more than one irreducible representation, but we do have some information about their number. Important for our purposes is that this parametrization clearly distinguishes tempered and nontempered representations. These results were inspired by unpublished work of Delorme and Opdam [DeOp2] on affine Hecke algebras.

For $\xi=(P, A, \omega, \chi) \in \Xi$ we define

$$
\begin{aligned}
v(\xi) & =\log |\chi|, & & \Sigma(\xi)=\left\{\alpha \in \Sigma(P, A) \mid\langle\nu(\xi), \alpha\rangle_{0}=0\right\}, \\
M(\xi) & =Z_{G}(A(\xi)), & A(\xi) & =\{a \in A \mid \alpha(a)=1 \text { for all } \alpha \in \Sigma(\xi)\}, \\
P(\xi) & =P M(\xi), & \omega(\xi) & =I_{P \cap M(\xi)}^{M(\xi)}\left(\omega \otimes \chi|\chi|^{-1}\right) .
\end{aligned}
$$

By [Wal], Lemme III.2.3, $\omega(\xi)$ is a tempered pre-unitary $M(\xi)$-representation. For $\xi \in \Xi_{u}$ we simply have

$$
v(\xi)=0, \quad \Sigma(\xi)=\Sigma(P, A), \quad A(\xi)=A_{G}, \quad P(\xi)=M(\xi)=G, \quad \omega(\xi)=I(\xi) .
$$

For general $\xi$ these objects are designed to divide parabolic induction into stages, like in $[\mathrm{KnVo}], \S \mathrm{XI} .9$. The first stage corresponds to the unitary part of $\chi$ and the second 
stage to its absolute value. This is possible since

$$
\begin{aligned}
I_{P(\xi)}^{G} & (|\chi| \otimes \omega(\xi)) \\
& \cong \operatorname{Ind}_{P(\xi)}^{G}\left(\delta_{P(\xi)}^{1 / 2} \otimes|\chi| \otimes \omega(\xi)\right) \\
& \cong \operatorname{Ind}_{P(\xi)}^{G}\left(\delta_{P(\xi)}^{1 / 2} \otimes|\chi| \otimes \operatorname{Ind}_{P \cap M(\xi)}^{M(\xi)}\left(\delta_{P \cap M(\xi)}^{1 / 2} \otimes \chi|\chi|^{-1} \otimes \omega\right)\right) \\
& \cong \operatorname{Ind}_{P M(\xi)}^{G}\left(\delta_{P M(\xi)}^{1 / 2} \otimes \operatorname{Ind}_{P \cap M(\xi)}^{M(\xi)}\left(\delta_{P \cap M(\xi)}^{1 / 2} \otimes \chi \otimes \omega\right)\right) \\
& \cong \operatorname{Ind}_{P M(\xi)}^{G}\left(\operatorname{Ind}_{P \cap(\xi)}^{M(\xi)}\left(\delta_{P M(\xi)}^{1 / 2} \otimes \delta_{P \cap M(\xi)}^{1 / 2} \otimes \chi \otimes \omega\right)\right) \\
& \cong \operatorname{Ind}_{P}^{G}\left(\delta_{P}^{1 / 2} \otimes \chi \otimes \omega\right)=I(\xi) .
\end{aligned}
$$

Clearly we can transfer the positivity condition from Langlands data to induction data. We say that $\xi=(P, A, \omega, \chi) \in \Xi^{+}$if $(P, A)$ is standard and $\log |\chi| \in \bar{a}^{*,+}$. This choice of a "positive cone" is justified by the following result.

Lemma 2.13. Every $\xi \in \Xi$ is $\mathcal{W}$-associate to an element of $\Xi^{+}$. If $\xi_{1}, \xi_{2} \in \Xi^{+}$are W-associate, then the objects $\Sigma\left(\xi_{i}\right), A\left(\xi_{i}\right), M\left(\xi_{i}\right), P\left(\xi_{i}\right)$ and $v\left(\xi_{i}\right)$ are the same for $i=1$ and $i=2$, while $\omega\left(\xi_{1}\right)$ and $\omega\left(\xi_{2}\right)$ are equivalent $M\left(\xi_{i}\right)$-representations.

Proof. As we noted before, every parabolic pair is conjugate to a standard one. By [Hum], Section 1.15 , every $W_{0}$-orbit in $a_{0}^{*}$ contains a unique point in a positive chamber $a^{*,+}$ (for a unique $A \subset A_{0}$ ). This proves the first claim and it also shows that

$$
\log \left|\chi_{1}\right|=\log \left|\chi_{2}\right| \in a_{0}^{*} \text {. }
$$

Hence the $v$ 's, $\Sigma$ 's, $A$ 's, $P$ 's and $M$ 's are the same for $i=1$ and $i=2$. If now $w \in \mathcal{W}$ is such that $w \omega_{1} \cong \omega_{2}$, then by Theorem 2.5 , applied to $M\left(\xi_{i}\right)$, there is a unitary intertwiner between $\omega\left(\xi_{1}\right)$ and $\omega\left(\xi_{2}\right)$.

The link between these positive induction data and the Langlands classification is easily provided:

Proposition 2.14. Suppose that $\xi=(P, A, \omega, \chi) \in \Xi^{+}$.

a) Let $\tau$ be an irreducible direct summand of $\omega(\xi)$. Then $(P(\xi), A(\xi), \tau, \nu(\xi)) \in$ $\Lambda^{+}$.

b) The irreducible quotients of $I(\xi)$ are exactly the modules $J(P(\xi), A(\xi), \tau, \nu(\xi))$ with $\tau$ as above, and these are tempered if and only if $\xi \in \Xi_{u}$.

c) The functor $I_{P(\xi)}^{G}$ induces an isomorphism $\operatorname{End}_{M(\xi)}(\omega(\xi)) \cong \operatorname{End}_{G}(I(\xi))$.

Proof. a) follows directly from the definitions in (2.30).

b) follows from a) and Theorem 2.10.

c) Let $\sigma$ be an irreducible direct summand of $\omega(\xi)$, which is not equivalent to $\tau$ as a $M(\xi)$-representation. By Lemma 2.11.b,

$$
J(P(\xi), A(\xi), \tau, \nu(\xi)) \notin \mathrm{JH}(I(P(\xi), A(\xi), \sigma, \nu(\xi))) .
$$


The proof of (2.28), with $I(P(\xi), A(\xi), \sigma, v(\xi))$ in the role of $M(\lambda)$, shows that

$$
\operatorname{Hom}_{G}(I(P(\xi), A(\xi), \tau, \nu(\xi)), I(P(\xi), A(\xi), \sigma, v(\xi)))=0
$$

Now the result follows easily from Lemma 2.11.c.

As announced, we can parametrize $\operatorname{Irr}(G)$ with our induction data.

Theorem 2.15. For every $\pi \in \operatorname{Irr}(G)$ there exists a unique association class $\mathcal{W}(P, A, \omega, \chi) \in \Xi / \mathcal{W}$ such that the following equivalent statements hold:

a) For some $\xi^{+} \in \mathcal{W}(P, A, \omega, \chi) \cap \Xi^{+}, \pi$ is equivalent to an irreducible quotient of $I\left(\xi^{+}\right)$.

b) $\pi \in \mathrm{JH}(I(P, A, \omega, \chi))$ and $\left\|\mathrm{cc}_{M}(\omega)\right\|$ is maximal with respect to this property.

Proof. a) Let $\left(P_{\pi}, A_{\pi}, \sigma, v\right)$ be the Langlands datum associated to $\pi$. Write $\mathcal{W}^{H}$, $\Xi^{H}$, etc. for $\mathcal{W}, \Xi$ but now corresponding to $H=M_{\pi}=Z_{G}\left(A_{\pi}\right)$ instead of $G$. By Theorem 2.6, applied to $H$, there exists a unique association class

$$
W^{H} \xi^{H}=W^{H}\left(P_{F} \cap H, A_{F}, \omega^{H}, \chi^{H}\right) \in \Xi_{u}^{H} / W^{H}
$$

such that $\sigma$ is a direct summand of $I^{H}(\xi)=I_{P_{F} \cap H}^{H}\left(\omega^{H} \otimes \chi^{H}\right)$. Put

$$
\xi^{+}=\left(P_{F}, A_{F}, \omega^{H}, \chi^{H} \cdot \chi_{\nu}\right) \in \Xi^{+} \text {. }
$$

By Proposition 2.14.b, $\pi \in \mathrm{JH}\left(I\left(\xi^{+}\right)\right)$and by Lemma 2.13 and Theorem 2.6 the class $\mathcal{W} \xi^{+} \in \Xi / \mathcal{W}$ is unique for this property.

b) We may assume that $\xi=(P, A, \omega, \chi) \in \Xi^{+}$in view of Corollary 2.7 and Lemma 2.13. Suppose that $\pi$ is not equivalent to a quotient of $I(\xi)$ and let $\tau$ be an irreducible summand of $\omega(\xi)$ such that $\pi \in \mathrm{JH}(I(\lambda))$ with $\lambda=(P(\xi), A(\xi), \tau, v(\xi)) \in$ $\Lambda^{+}$. By Lemma 2.12 and (2.29),

$$
\left\|c_{M_{F}}\left(\omega^{H}\right)\right\|=\left\|\operatorname{cc}_{M_{\pi}}(\sigma)\right\|<\left\|\operatorname{cc}_{M(\xi)}(\tau)\right\|=\left\|\operatorname{cc}_{M}(\omega)\right\| .
$$

This contradiction proves that $\pi$ must be equivalent to a quotient of $I(\xi)$. Hence $\xi$ satisfies the condition from a), which shows that $\mathcal{W} \xi$ is unique. In particular conditions a) and b) are equivalent.

Every standard triple $(P, A, \omega)$ gives a series of finite length $G$-representations $I(P, A, \omega, \chi)$, parametrized by $\chi \in X_{\mathrm{nr}}(M)$. Every such series lies in a single Bernstein component. Let $\operatorname{Irr}(G)_{(P, A, \omega)}$ denote the set of all $\pi \in \operatorname{Irr}(G)$ which are equivalent to an irreducible quotient of $I(\xi)$, for some $\xi \in \Xi^{+} \cap \mathcal{W}\left(P, A, \omega, X_{\mathrm{nr}}(M)\right)$. Theorem 2.15 tells us that these subsets form a partition of $\operatorname{Irr}(G)$. We note that

$$
\operatorname{Irr}^{t}(G)_{(P, A, \omega)}:=\operatorname{Irr}(G)_{(P, A, \omega)} \cap \operatorname{Irr}^{t}(G)
$$


consists of the direct summands of the $I(P, A, \omega, \chi)$ with $\chi \in X_{\mathrm{unr}}(M)$. As a topological space $\operatorname{Irr}(G)_{(P, A, \omega)}$ is usually not Hausdorff, since certain $\chi \in X_{\mathrm{nr}} M$ carry more than one point. The following result shows that the singularities depend only on the $\mathcal{W}$-action and can therefore already be detected on $\operatorname{Irr}^{t}(G)_{(P, A, \omega)}$.

Lemma 2.16. Suppose that $t \mapsto \xi_{t}=\left(P, A, \omega, \chi_{t}\right)$ is a path in $\Xi$, and that $\mathcal{W}_{\xi}:=$ $\left\{w \in \mathcal{W} \mid w\left(\xi_{t}\right)=\xi_{t}\right\}$ does not depend on $t$. Then $\left|J H\left(I\left(\xi_{t}\right)\right) \cap \operatorname{Irr}(G)_{(P, A, \omega)}\right|$ does not depend on $t$.

Proof. For $\chi_{t}$ moving within $X_{\text {unr }}(M)$ this property can be read off from Theorem 2.9. Take $n \in \mathcal{W}_{\xi}$. According to Theorem 2.5 the intertwiner $I(n, \omega \otimes \chi)$ extends holomorphically to a tubular neighborhood $U$ of $X_{\mathrm{unr}}(M)$ in $X_{\mathrm{nr}}(M)$. Hence the desired result also holds for paths with $\chi_{t} \in U$ for all $t$.

Now consider any path as in the statement. For every $t_{0}$ there is an $r \in(-1,0]$ and a neighborhood $T$ of $t_{0}$ such that

$$
\chi_{t}^{\prime}:=\chi_{t}\left|\chi_{t}\right|^{r} \in U \quad \text { for all } t \in T .
$$

By Proposition 2.14.b,

$$
\left|\operatorname{JH}\left(I\left(\xi_{t}\right)\right) \cap \operatorname{Irr}(G)_{(P, A, \omega)}\right|=\left|\operatorname{JH}\left(I\left(\xi_{t}^{\prime}\right)\right) \cap \operatorname{Irr}(G)_{(P, A, \omega)}\right| .
$$

We just saw that the right-hand side is independent of $t \in T$, hence so is the left-hand side.

With these partitions of $\operatorname{Irr}(G)$ and $\operatorname{Irr}^{t}(G)$ we can construct filtrations of corresponding algebras, which will be essential in the next chapter.

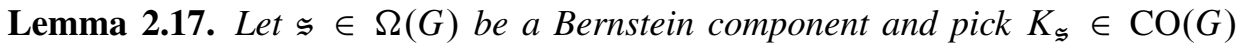
as in Proposition 2.1.b. There exist standard triples $\left(P_{i}, A_{i}, \omega_{i}\right)$ and filtrations by two-sided ideals

$$
\begin{aligned}
& \mathscr{H}\left(G, K_{\mathfrak{s}}\right)^{\mathfrak{S}}=\mathscr{H}_{0}^{\mathfrak{s}} \supset \mathscr{H}_{1}^{\mathfrak{s}} \supset \cdots \supset \mathscr{H}_{n_{\mathfrak{S}}}^{\mathfrak{s}}=0, \\
& \delta\left(G, K_{\mathfrak{s}}\right)^{\mathfrak{F}}={S_{0}^{\mathfrak{F}}} \supset{S_{1}^{\mathfrak{F}}} \supset \cdots \supset{S_{n_{\mathfrak{s}}}^{\mathfrak{s}}}=0
\end{aligned}
$$

such that

a. $\mathscr{H}_{i}^{\mathfrak{s}} \subset S_{i}^{\mathfrak{F}}$,

b. $\operatorname{Prim}\left(\mathscr{H}_{i-1}^{\mathfrak{s}} / \mathcal{H}_{i}^{\mathfrak{s}}\right) \cong \operatorname{Irr}(G)_{\left(P_{i}, A_{i}, \omega_{i}\right)}$

c. $\operatorname{Prim}\left({S_{i-1}^{\mathfrak{F}}}^{\mathfrak{S}} / \mathcal{S}_{i}^{\mathfrak{S}}\right) \cong \operatorname{Irr}^{t}(G)_{\left(P_{i}, A_{i}, \omega_{i}\right)}$.

Proof. By (2.11), $\delta\left(G, K_{\mathfrak{s}}\right)^{\mathfrak{F}}$ is a direct summand of $\delta\left(G, K_{\mathfrak{S}}\right)$, so Theorem 2.9 assures the existence of finitely many (say $\left.n_{\mathfrak{\Im}}\right)$ standard triples $\left(P_{i}, A_{i}, \omega_{i}\right)$ such that

$$
\delta\left(G, K_{\mathfrak{s}}\right)^{\mathfrak{s}} \cong \bigoplus_{i}\left(C^{\infty}\left(X_{\mathrm{unr}}\left(M_{i}\right)\right) \otimes L\left(\omega_{i}, P_{i}\right)^{K_{\mathfrak{5}} \times K_{\mathfrak{5}}}\right)^{w_{i}} .
$$


In view of Theorem 2.2, $\operatorname{Prim}\left(\mathscr{H}\left(G, K_{\mathfrak{s}}\right)^{\mathfrak{s}}\right)$ is a union of series corresponding to standard triples. Looking at the unitary parts of these series and at (2.33), we find that

$$
\operatorname{Prim}\left(\mathscr{H}\left(G, K_{\mathfrak{s}}\right)^{\mathfrak{s}}\right) \cong \bigcup_{i} \operatorname{Irr}(G)_{\left(P_{i}, A_{i}, \omega_{i}\right)} .
$$

Number the triples $\left(P_{i}, A_{i}, \omega_{i}\right)$ from 1 to $n_{\mathfrak{s}}$ such that

$$
\left\|\operatorname{cc}_{M_{j}}\left(\omega_{j}\right)\right\| \geq\left\|\operatorname{cc}_{M_{i}}\left(\omega_{i}\right)\right\| \quad \text { if } j \leq i .
$$

We define

$$
\begin{aligned}
\mathscr{H}_{i}^{\mathfrak{s}} & =\left\{h \in \mathcal{H}\left(G, K_{\mathfrak{s}}\right)^{\mathfrak{F}} \mid \pi(h)=0 \text { for all } \pi \in \operatorname{Irr}(G)_{\left(P_{j}, A_{j}, \omega_{j}\right)}, j \leq i\right\}, \\
\mathcal{S}_{i}^{\mathfrak{S}} & =\left\{h \in \mathcal{S}\left(G, K_{\mathfrak{s}}\right)^{\mathfrak{S}} \mid \pi(h)=0 \text { for all } \pi \in \operatorname{Irr}^{t}(G)_{\left(P_{j}, A_{j}, \omega_{j}\right)}, j \leq i\right\} .
\end{aligned}
$$

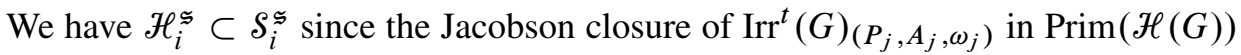
contains $\operatorname{Irr}(G)_{\left(P_{j}, A_{j}, \omega_{j}\right)}$. From (2.33) we get

$$
{S_{i-1}^{\mathfrak{F}}}^{\mathfrak{s}} / \mathcal{S}_{i}^{\mathfrak{F}} \cong\left(C^{\infty}\left(X_{\mathrm{unr}}\left(M_{i}\right)\right) \otimes L\left(\omega_{i}, P_{i}\right)^{K_{\mathfrak{5}} \times K_{\mathfrak{5}}}\right)^{\mathfrak{W}_{i}},
$$

which shows that c) holds. We claim that

$$
\bigcup_{j \leq i} \operatorname{Irr}(G)_{\left(P_{j}, A_{j}, \omega_{j}\right)}
$$

is closed in the Jacobson topology of $\mathscr{H}\left(G, K_{\mathfrak{s}}\right)^{\mathfrak{s}}$. Its Jacobson closure consists of all irreducible subquotients $\pi$ of $I\left(\xi_{j}\right)=I\left(P_{j}, A_{j}, \omega_{j}, \chi\right)$ for any $j \leq i$ and $\chi \in$ $X_{\text {nr }}\left(M_{j}\right)$. Suppose that $\pi \notin \operatorname{Irr}(G)_{\left(P_{j}, A_{j}, \omega_{j}\right)}$. By Theorem 2.15, $\pi \in \operatorname{Irr}(G)_{(P, A, \omega)}$ for some standard triple with $\left\|\operatorname{cc}_{M}(\omega)\right\|>\left\|\operatorname{cc}_{M_{j}}\left(\omega_{j}\right)\right\|$. In view of (2.34) we must have $w(P, A, \omega)=\left(P_{n}, A_{n}, \omega_{n}\right)$ for some $w \in \mathcal{W}$ and $n \in \mathbb{N}$. Then

$$
\left\|\operatorname{cc}_{M_{n}}\left(\omega_{n}\right)\right\|=\left\|\operatorname{cc}_{M}(\omega)\right\|>\left\|\operatorname{cc}_{M_{j}}\left(\omega_{j}\right)\right\|,
$$

so $n<j \leq i$, proving our claim. Consequently

$$
\operatorname{Prim}\left(\mathcal{H}_{i}^{\mathfrak{s}}\right) \cong \bigcup_{j>i} \operatorname{Irr}(G)_{\left(P_{j}, A_{j}, \omega_{j}\right)},
$$

which easily implies b).

\section{The noncommutative geometry of reductive $p$-adic groups}

3.1. Periodic cyclic homology. The results of Sections 1 and 2 enable us to compare the K-theory and the periodic cyclic homology of reductive $p$-adic groups. Before proceeding we recall that $\mathrm{HP}_{*}$ is continuous in certain situations. Here and in the next results we will freely use the notations from Section 2. 
Theorem 3.1. a) Suppose that $A=\lim _{i \rightarrow \infty} A_{i}$ is an inductive limit of algebras and that there exists $N \in \mathbb{N}$ such that $\mathrm{HH}_{n}\left(A_{i}\right)=0$ for all $n>N$ and for all $i$. Then $\mathrm{HP}_{*}(A) \cong \lim _{i \rightarrow \infty} \mathrm{HP}_{*}\left(A_{i}\right)$.

b) Suppose that $B=\lim _{i \rightarrow \infty} B_{i}$ is a strict inductive limit of nuclear Fréchet algebras and that there exists $N \in \mathbb{N}$ such that $\mathrm{HH}_{n}\left(B_{i}, \widehat{\otimes}\right)=0$ for all $n>N$ and for all $i$. Then $\mathrm{HP}_{*}\left(B, \hat{\otimes}_{\mathbb{C}}\right) \cong \lim _{i \rightarrow \infty} \operatorname{HP}_{*}\left(B_{i}, \hat{\otimes}\right)$.

Proof. According to [Mey3], Theorem 1.93, the completed bornological tensor product agrees with Grothendieck's completed inductive tensor product for strict inductive limits of nuclear Fréchet spaces. This identifies b) with [BrPl], Theorem 3. Part a) is just the simpler algebraic version of this result, which can also be found in [Nis], Proposition 2.2.

Theorem 3.2. Let $\mathfrak{s} \in \Omega(G)$ be a Bernstein component and let $K_{\mathfrak{s}} \in \mathrm{CO}(G)$ be as in Proposition 2.1.b.

a) The Chern character for $S\left(G, K_{\mathfrak{s}}\right)^{\mathfrak{s}}$ induces an isomorphism

$$
K_{*}\left(C_{r}(G)^{\mathfrak{s}}\right) \otimes_{\mathbb{Z}} \mathbb{C} \rightarrow \operatorname{HP}_{*}\left(S(G)^{\mathfrak{s}}, \hat{\otimes}_{\mathbb{C}}\right)
$$

b) The direct sum of these maps, over all $\mathfrak{s} \in \Omega(G)$, is a natural isomorphism

$$
K_{*}\left(C_{r}(G)\right) \otimes_{\mathbb{Z}} \mathbb{C} \rightarrow \mathrm{HP}_{*}\left(S(G), \hat{\otimes}_{\mathbb{C}}\right)
$$

Proof. By (2.33) and Theorem 1.9 the Chern character

$$
\operatorname{ch} \otimes \operatorname{id}_{\mathbb{C}}: K_{*}\left(S\left(G, K_{\mathfrak{s}}\right)^{\mathfrak{s}}\right) \otimes_{\mathbb{Z}} \mathbb{C} \rightarrow \operatorname{HP}_{*}\left(S\left(G, K_{\mathfrak{s}}\right)^{\mathfrak{F}}\right)
$$

is an isomorphism. Furthermore there are natural isomorphisms

$$
\begin{aligned}
K_{*}\left(C_{r}^{*}(G)\right) & \left.\cong K_{*} \underset{\mathfrak{S}}{\underset{\mathfrak{l i m} \in \mathbb{S}}{\longrightarrow}} C_{r}^{*}(G)^{\mathfrak{s}}\right) \\
& \cong \bigoplus_{\mathfrak{s} \in \Omega(G)} K_{*}\left(C_{r}^{*}(G)^{\mathfrak{s}}\right) \\
& \cong \bigoplus_{\mathfrak{s} \in \Omega(G)} \underset{K \in \operatorname{CO}(G)}{\lim _{\boldsymbol{C}}} K_{*}\left(C_{r}^{*}(G, K)^{\mathfrak{s}}\right) \\
& \cong \bigoplus_{\mathfrak{s} \in \Omega(G)} K_{*}\left(C_{r}^{*}\left(G, K_{\mathfrak{s}}\right)^{\mathfrak{s}}\right) \\
& \cong \bigoplus_{\mathfrak{s} \in \Omega(G)} K_{*}\left(S\left(G, K_{\mathfrak{s}}\right)^{\mathfrak{s}}\right)
\end{aligned}
$$

Here we used respectively (2.13), (2.9), Proposition 2.1.d and that $K_{*}$ is invariant for 
passing to holomorphically closed dense Fréchet subalgebras. Similarly,

$$
\begin{aligned}
\mathrm{HP}_{*}\left(S(G), \hat{\otimes}_{\mathbb{C}}\right) & \cong \underset{K \in \lim _{K}}{\longrightarrow} \mathrm{HP}_{*}(S(G, K), \hat{\otimes}) \\
& \cong \bigoplus_{\mathfrak{s} \in \Omega(G)} \lim _{K \in \mathrm{CO}(G)} \operatorname{HP}_{*}\left(S(G, K)^{\mathfrak{s}}, \hat{\otimes}\right) \\
& \cong \bigoplus_{\mathfrak{s} \in \Omega(G)} \operatorname{HP}_{*}\left(S(G)^{\mathfrak{s}}, \hat{\otimes}_{\mathbb{C}}\right) \\
& \cong \bigoplus_{\mathfrak{s} \in \Omega(G)} \operatorname{HP}_{*}\left(S\left(G, K_{\mathfrak{s}}\right)^{\mathfrak{s}}, \hat{\otimes}\right)
\end{aligned}
$$

For the first and third isomorphisms we need Theorem 3.1.b, which we may indeed apply due to Corollary 2.4.b. The second and fourth isomorphisms rely on Proposition 2.1.c.

Finally we combine the last lines of (3.2) and (3.3) with (3.1).

Altogether this theorem involves quite a few steps, but one is always guided by the general principle that algebras with the same spectrum should have closely related invariants.

Next we will prove the comparison theorem for the periodic cyclic homology of reductive $p$-adic groups. This result was suggested in [BHP3], Conjecture 8.9, and in [ABP], Section 4. It is more difficult than Theorem 3.2, precisely because the above principle does not apply. We remark that Nistor [Nis], Theorem 4.2, already gave a rather explicit description of $\mathrm{HP}_{*}(\mathscr{H}(G))$.

Theorem 3.3. The inclusions $\mathscr{H}(G)^{\mathfrak{s}} \rightarrow S(G)^{\mathfrak{s}}$ for $\mathfrak{s} \in \Omega(G)$ induce isomorphisms

$$
\begin{aligned}
& \mathrm{HP}_{*}\left(\mathscr{H}(G)^{\mathfrak{s}}\right) \sim \mathrm{HP}_{*}\left(S(G)^{\mathfrak{s}}, \hat{\otimes}_{\mathbb{C}}\right), \\
& \mathrm{HP}_{*}(\mathscr{H}(G)) \stackrel{\sim}{\longrightarrow} \operatorname{HP}_{*}\left(S(G), \hat{\otimes}_{\mathbb{C}}\right) .
\end{aligned}
$$

Proof. It follows from Theorem 3.1.a, Corollary 2.4.a and Proposition 2.1.b that

$$
\begin{aligned}
\mathrm{HP}_{*}(\mathscr{H}(G)) & \cong \bigoplus_{\mathfrak{s} \in \Omega(G)} \mathrm{HP}_{*}\left(\mathscr{H}(G)^{\mathfrak{s}}\right) \\
& \cong \bigoplus_{\mathfrak{s} \in \Omega(G)} \underset{K \in \mathrm{CO}(G)}{\lim } \operatorname{HP}_{*}\left(\mathscr{H}(G, K)^{\mathfrak{s}}\right) \\
& \cong \bigoplus_{\mathfrak{s} \in \Omega(G)} \operatorname{HP}_{*}\left(\mathscr{H}\left(G, K_{\mathfrak{s}}\right)^{\mathfrak{s}}\right)
\end{aligned}
$$

Together with (3.3) this reduces the proof to showing that for every Bernstein component $s \in \Omega(G)$ the inclusion

$$
\mathscr{H}\left(G, K_{\mathfrak{s}}\right)^{\mathfrak{s}} \rightarrow \delta\left(G, K_{\mathfrak{s}}\right)^{\mathfrak{s}}
$$

induces an isomorphism on periodic cyclic homology. Applying Lemma 1.3 to the filtrations from Lemma 2.17, this will follow from the next result. 
Proposition 3.4. Let $\mathscr{H}_{i}^{\mathfrak{s}}$ and ${S_{i}^{\mathfrak{F}}}_{\text {be }}$ as in (2.35). The inclusion map

$$
\mathscr{H}_{i-1}^{\mathfrak{S}} / \mathcal{H}_{i}^{\mathfrak{S}} \rightarrow S_{i-1}^{\mathfrak{S}} / S_{i}^{\mathfrak{S}}
$$

induces an isomorphism on periodic cyclic homology.

Proof. We would like to copy the proof of Theorem 1.10 with $\Gamma=\mathcal{W}_{i}$ and

$$
\begin{aligned}
A_{\mathrm{alg}} & =\mathscr{H}_{i-1}^{\mathfrak{s}} / \mathscr{H}_{i}^{\mathfrak{s}}, & X & =X_{\mathrm{nr}}\left(M_{i}\right), \\
A_{\text {smooth }} & =S_{i-1}^{\mathfrak{F}} / S_{i}^{\mathfrak{F}}, & X^{\prime} & =X_{\mathrm{unr}}\left(M_{i}\right) .
\end{aligned}
$$

By Lemmas 2.16 and 2.17 the primitive ideal spectra fit into the framework of Section 1.4. On the smooth side everything works fine, but on the algebraic side we have to take into account that in general

- the intertwiners $u_{w}\left(w \in \mathcal{W}_{i}\right)$ are only rational on $X_{\mathrm{nr}}\left(M_{i}\right)$,

- the center of $\mathscr{H}_{i-1}^{\mathfrak{s}} / \mathscr{H}_{i}^{\mathfrak{s}}$ is "too small" in the sense that it does not contain all polynomial functions on $X_{\mathrm{nr}}\left(M_{i}\right) / W_{i}$.

In fact every element of

$$
Z\left(\mathscr{H}_{i-1}^{\mathfrak{s}} / \mathscr{H}_{i}^{\mathfrak{s}}\right) \subset \mathcal{O}\left(X_{\mathrm{nr}}\left(M_{i}\right) / W_{i}\right)
$$

necessarily vanishes on the singularities of the intertwiners, because these points of $X_{\mathrm{nr}}\left(M_{i}\right)$ carry representations from $\operatorname{Irr}(G)_{\left(P_{j}, A_{j}, \omega_{j}\right)}$ with $j<i$.

Let $\mathscr{L}$ be the collection of all the irreducible components of all the $X^{H}$, with $H$ running over all subsets of $\mathcal{W}_{i}$. We note that these are all of the form $\chi T$, with $\chi \in X_{\text {unr }}\left(M_{i}\right)$ and $T$ an algebraic subtorus of $X_{\mathrm{nr}}\left(M_{i}\right)$. We write $T_{u}=T \cap X_{\text {unr }}\left(M_{i}\right)$. Let $\mathscr{L}_{p}$ be the subset of $\mathscr{L}$ consisting of elements of dimension $\leq p$, and define the $\mathcal{W}_{i}$-stable subvarieties

$$
\begin{aligned}
& X_{p}:=\bigcup_{\chi T \in \mathscr{L}_{p}} \chi T, \\
& X_{p}^{\prime}:=X_{p} \cap X_{\mathrm{unr}}\left(M_{i}\right)=\bigcup_{\chi T \in \mathscr{L}_{p}} \chi T_{u} .
\end{aligned}
$$

Let $V$ be the finite dimensional vector space $I_{P_{i}}^{G}\left(E_{i}\right)^{K_{\Im}}$ and consider the ideals

$$
\begin{aligned}
I_{p} & :=\left\{h \in \mathscr{H}_{i-1}^{\mathfrak{s}} / \mathscr{H}_{i}^{\mathfrak{s}} \mid I\left(P_{i}, A_{i}, \omega_{i}, \chi\right)(h)=0 \text { for all } \chi \in X_{p}\right\}, \\
J_{p} & :=\left\{h \in S_{i-1}^{\mathfrak{F}} / S_{i}^{\mathfrak{F}} \mid I\left(P_{i}, A_{i}, \omega_{i}, \chi\right)(h)=0 \text { for all } \chi \in X_{p}^{\prime}\right\} .
\end{aligned}
$$

It follows from (2.36) that

$$
J_{p-1} / J_{p}=C_{0}^{\infty}\left(X_{p}^{\prime}, X_{p-1}^{\prime} ; \operatorname{End}(V)\right)^{\mathfrak{W}_{i}}, \quad I_{p-1} / I_{p} \subset \mathcal{O}_{0}\left(X_{p}, X_{p-1} ; \operatorname{End}(V)\right)^{\mathfrak{W}_{i}} .
$$

The Jacobson topology makes Prim $\left(A_{\text {alg }} / I_{p}\right)$ into a complex scheme, which need not be separable. Let

$$
\theta_{p}: \operatorname{Prim}\left(A_{\mathrm{alg}} / I_{p}\right) \rightarrow X_{p} / \mathcal{W}_{i}
$$


be the natural morphism. Take $C \in \mathscr{L}_{p}$ and consider $Y_{C}=\theta_{p}^{-1}\left(\left(\mathcal{W}_{i} C\right) / W_{i}\right)$. From Lemma 2.16 we see that $Y_{C}$ is a disjoint union of copies of $\left(W_{i} C\right) / W_{i}$, of which certain subvarieties are given a higher multiplicity. Moreover, the only thing that can cause inseparability on $\operatorname{Prim}\left(A_{\mathrm{alg}} / I_{p}\right)$ is a jump in the isotropy group of $W_{i}$ acting on $C$. By construction of $X_{p}$ this implies that all inseparable points lie in $\theta_{p}^{-1}\left(X_{p-1} / W_{i}\right)$, so the corresponding primitive ideals contain $I_{p-1} / I_{p}$.

We define $Y_{p}$ as the (maximal) separable quotient of $\operatorname{Prim}\left(A_{\mathrm{alg}} / I_{p}\right)$. From the above description we see that it is a complex algebraic variety on which $\theta_{p}$ is well defined. Let $Z_{p}$ be its closed subvariety corresponding to $X_{p-1} / W_{i}$. Then

$$
\operatorname{Prim}\left(I_{p-1} / I_{p}\right)=Y_{p} \backslash Z_{p},
$$

and this would agree with (1.21) if all the intertwiners were polynomial. We have

$$
\begin{aligned}
Y_{p}^{\prime} & :=Y_{p} \cap \theta_{p}^{-1}\left(X_{p}^{\prime} / W_{i}\right)=\text { Hausdorff quotient of } \operatorname{Prim}\left(A_{\text {smooth }} / J_{p}\right), \\
Z_{p}^{\prime} & :=Z_{p} \cap \theta_{p}^{-1}\left(X_{p}^{\prime} / W_{i}\right)=\left\{J / \sim \in Y_{p}^{\prime} \mid Z\left(J_{p-1} / J_{p}\right) \subset J\right\}, \\
Y_{p}^{\prime} \backslash Z_{p}^{\prime} & =\operatorname{Prim}\left(J_{p-1} / J_{p}\right)=\operatorname{Prim}\left(Z\left(J_{p-1} / J_{p}\right)\right) .
\end{aligned}
$$

With these notations the singularities of the $u_{w}$ form a closed subvariety of $Y_{p}$, disjoint from $Y_{p}^{\prime}$. From p. 518 we know that there are natural isomorphisms

$$
\operatorname{HP}_{*}\left(J_{p-1} / J_{p}\right) \leftarrow \operatorname{HP}_{*}\left(Z\left(J_{p-1} / J_{p}\right)\right)=\operatorname{HP}_{*}\left(\widetilde{C}_{0}^{\infty}\left(Y_{p}^{\prime}, Z_{p}^{\prime}\right)\right) \cong H^{[*]}\left(Y_{p}^{\prime}, Z_{p}^{\prime}\right) .
$$

For our comparison we will construct something similar on the algebraic side.

Lemma 3.5. There exists a natural map

$$
\mathrm{HP}_{*}\left(I_{p-1} / I_{p}\right) \rightarrow \mathrm{HP}_{*}\left(\mathcal{O}_{0}\left(Y_{p}, Z_{p}\right)\right) .
$$

Proof. The main problem is the absence of a natural algebra homomorphism between $I_{p-1} / I_{p}$ and $\mathcal{O}_{0}\left(Y_{p}, Z_{p}\right)$. As a substitute we will use the generalized trace map on the periodic cyclic bicomplex.

Let $C$ be an irreducible component of $X_{p}$, which is not contained in $X_{p-1}$. Pick any point $\chi \in C \backslash\left(C \cap X_{p-1}\right)$ and define

$$
\mathcal{W}_{C}:=\{w \in \mathcal{W} \mid w \chi=\chi\} .
$$

This depends only on $C$, not on the choice of $\chi$. Since $C$ is of the form "algebraic subtorus translated by a unitary element", $C \cap X_{\text {unr }}\left(M_{i}\right)$ is a (nonempty) real form of $C$. According to Theorem 2.5 the intertwiners $I(w, \omega \otimes \chi)$ with $w \in \mathcal{W}_{C}$ are regular on $C \cap X_{\text {unr }}\left(M_{i}\right)$, so they are regular on a nonempty Zariski-open subset of $C$. But $I(w, \omega \otimes \chi) \in \mathrm{GL}\left(\operatorname{End}_{\mathbb{C}}(V)\right)$ has finite order for all $\chi \in C$, so it is in fact regular on the whole of $C$. In particular, for every $\chi \in C$ there is a canonical decomposition of $V$ into isotypical projective $W_{C}$-representations:

$$
I\left(P_{i}, A_{i}, \omega_{i}, \chi\right)^{K_{\mathfrak{s}}}=V=V_{1}^{\chi} \oplus \cdots \oplus V_{n_{C}}^{\chi} .
$$


Since $\chi \mapsto I(w, \omega \otimes \chi)$ is polynomial on $C$, the type of $V$ as a projective $W_{C}$ representation is independent of $\chi \in C$. Moreover the corresponding projections $p(C, n, \chi) \in \operatorname{End}_{\mathbb{C}}(V)$ are polynomial in $\chi$.

For $\chi \in C \cap\left(X_{p}^{\prime} \backslash X_{p-1}^{\prime}\right)$ Theorem 2.9 assures that

$$
\operatorname{End}_{\mathcal{W}_{C}}\left(I\left(P_{i}, A_{i}, \omega_{i}, \chi\right)^{K_{\mathfrak{S}}}\right)=\left\{I\left(P_{i}, A_{i}, \omega_{i}, \chi\right)(h) \mid h \in \mathcal{S}_{i-1}^{\mathfrak{s}} / \mathcal{S}_{i}^{\mathfrak{F}}\right\},
$$

so the summands of (3.7) are in bijection with the irreducible constituents of the $\mathscr{H}_{i-1}^{\mathfrak{s}} / \mathscr{H}_{i}^{\mathfrak{s}}$-module $I\left(P_{i}, A_{i}, \omega_{i}, \chi\right)^{K_{\mathfrak{s}}}$. Together with Lemma 2.16 this shows that for every $\chi \in C \cap\left(X_{p} \backslash X_{p-1}\right)$ and every $\pi \in \mathrm{JH}_{A_{\text {alg }}}\left(I\left(P_{i}, A_{i}, \omega_{i}, \chi\right)^{K_{\mathfrak{s}}}\right)$ there is a unique direct summand of (3.7) in which $\pi$ appears. We remark that $\operatorname{JH}_{G}\left(I\left(P_{i}, A_{i}, \omega_{i}, \chi\right)\right)$ may be larger, but the additional elements do not belong to $\operatorname{Irr}(G)_{\left(P_{i}, A_{i}, \omega_{i}\right)}$.

For any $w \in \mathcal{W}_{i}$ and any $n \leq n_{C}$ the intertwiner $I(w, \omega \otimes \chi) \operatorname{maps}_{\operatorname{End}}\left(V_{n}^{\chi}\right)$ to $\operatorname{End}_{\mathbb{C}}\left(V_{n^{\prime}}^{w \chi}\right)$, for some isotypical component $V_{n^{\prime}}^{w \chi}$ of the projective $\left(w W_{C} w^{-1}\right)$ representation $I\left(P_{i}, A_{i}, \omega_{i}, w \chi\right)^{K_{\mathfrak{5}}}$. Write

$$
\tilde{Y}_{p}:=\bigsqcup_{C}\left\{1, \ldots, n_{C}\right\} \times C \quad, \quad \tilde{Z}_{p}:=\bigsqcup_{C}\left\{1, \ldots, n_{C}\right\} \times\left(C \cap X_{p-1}\right),
$$

and let $\mathcal{W}_{i}$ act on these spaces by sending $(n, C, \chi)$ to $\left(n^{\prime}, w C, w \chi\right)$. The above amounts to a bijection

$$
\operatorname{Prim}\left(I_{p-1} / I_{p}\right)=Y_{p} \backslash Z_{p} \rightarrow\left(\tilde{Y}_{p} \backslash \tilde{Z}_{p}\right) / W_{i} .
$$

Furthermore we can make $\mathcal{W}_{i}$ act on the algebra

$$
B_{p}:=\mathcal{O}_{0}\left(\tilde{Y}_{p}, \tilde{Z}_{p}\right) \otimes \operatorname{End}(V)
$$

by the natural combination of the actions on $I_{p-1} / I_{p}$ and on $\widetilde{Y}_{p}$. There is a morphism of finite type $Z\left(\mathcal{H}\left(G, K_{\mathfrak{s}}\right)^{\mathfrak{s}}\right)$-algebras

$$
\phi: I_{p-1} / I_{p} \rightarrow B_{p}^{w_{i}}, \quad \phi(h)(C, n, \chi)=p(C, n, \chi) I\left(P_{i}, A_{i}, \omega_{i}, \chi\right)(h) p(C, n, \chi) .
$$

By (3.8) the central subalgebra

$$
Z\left(B_{p}\right)^{W_{i}}=\mathcal{O}_{0}\left(\tilde{Y}_{p}, \tilde{Z}_{p}\right)^{W_{i}} \subset B_{p}^{W_{i}}
$$

satisfies

$$
\operatorname{Prim}\left(Z\left(B_{p}\right)^{W_{i}}\right)=\left(\tilde{Y}_{p} \backslash \tilde{Z}_{p}\right) / W_{i} \cong \operatorname{Prim}\left(I_{p-1} / I_{p}\right) .
$$

Now we are in the right position to apply the generalized trace map. Recall [Lod], Section 1.2 , that, for any algebra $B$, this is a collection of linear maps

$$
\operatorname{tr}_{m}:(B \otimes \operatorname{End}(V))^{\otimes m} \rightarrow B^{\otimes m}
$$

which together form chain maps on the standard complexes computing Hochschild and (periodic) cyclic homology. The tracial property can be formulated as

$$
\operatorname{tr}_{n} \circ \operatorname{Ad}(b)^{\otimes n}=\operatorname{tr}_{n}
$$


for all invertible elements $b$ in the multiplier algebra of $B$. The induced maps on homology are natural and inverse to the maps induced by the inclusion

$$
B \rightarrow B \otimes \operatorname{End}(V): b \mapsto e b e
$$

with $e \in \operatorname{End}(V)$ an idempotent of rank one. Consider the composition

$$
\operatorname{tr}_{m} \circ \phi^{\otimes m}:\left(I_{p-1} / I_{p}\right)^{\otimes m} \rightarrow Z\left(B_{p}\right)^{\otimes m} .
$$

In view of (3.9) and (2.22) the image is contained in $\left(\left(Z\left(B_{p}\right)\right)^{\otimes m}\right)^{w_{i}}$. The periodic cyclic bicomplex [Lod], Section 5.1, with terms $\left(\left(Z\left(B_{p}\right)\right)^{\otimes m}\right)^{w_{i}}$ has homology $\mathrm{HP}_{*}\left(Z\left(B_{p}\right)\right)^{W_{i}}$, because $W_{i}$ is finite and acts by algebra automorphisms. Thus we obtain a natural map

$$
\tau:=\operatorname{HP}_{*}(\operatorname{tr} \circ \phi): \operatorname{HP}_{*}\left(I_{p-1} / I_{p}\right) \rightarrow \operatorname{HP}_{*}\left(Z\left(B_{p}\right)\right)^{w_{i}} .
$$

By Theorem 1.6.a there are natural isomorphisms

$$
\begin{aligned}
\operatorname{HP}_{*}\left(Z\left(B_{p}\right)\right)^{w_{i}} \leftarrow \operatorname{HP}_{*}\left(Z\left(B_{p}\right)^{w_{i}}\right) & =\operatorname{HP}_{*}\left(\mathcal{O}_{0}\left(\tilde{Y}_{p}, \widetilde{Z}_{p}\right)^{w_{i}}\right) \\
& =\operatorname{HP}_{*}\left(\mathcal{O}_{0}\left(Y_{p}, Z_{p}\right)\right) \cong H^{[*]}\left(Y_{p}, Z_{p}\right) .
\end{aligned}
$$

The combination of (3.11) and (3.12) yields the required map.

Lemma 3.6. The map

$$
\operatorname{HP}_{*}\left(I_{p-1} / I_{p}\right) \rightarrow \operatorname{HP}_{*}\left(\mathcal{O}_{0}\left(Y_{p}, Z_{p}\right)\right)
$$

constructed in Lemma 3.5 is an isomorphism.

Proof. We will prove the equivalent statement that (3.11) is bijective.

For $q \in \mathbb{N}$, let $Y_{p, q} \subset \operatorname{Prim}\left(I_{p-1} / I_{p}\right)$ be the collection of all primitive ideals for which the corresponding irreducible $I_{p-1} / I_{p}$-module has dimension $\leq q$. According to [KNS], p. 328, these are Jacobson-closed subsets, and they give rise to the standard filtration

$$
I_{p-1} / I_{p}=L_{0} \supset L_{1} \supset \cdots \supset L_{\operatorname{dim} V}, \quad L_{q}:=\bigcap_{J \in Y_{p, q}} J .
$$

Since every irreducible $I_{p-1} / I_{p}$-module is a subquotient of $V, L_{\operatorname{dim} V}$ is the Jacobson radical of $I_{p-1} / I_{p}$. We would like to apply the results of [KNS] to (3.13), but this is not directly possible because $I_{p-1} / I_{p}$ does not have a unit. Therefore we consider the unital finite type algebra

$$
\begin{aligned}
A & :=Z\left(\mathscr{H}\left(G, K_{\mathfrak{s}}\right)^{\mathfrak{5}}\right) \oplus M_{2}\left(I_{p-1} / I_{p}\right), \\
(f \oplus h)\left(f^{\prime} \oplus h^{\prime}\right) & :=\left(f f^{\prime} \oplus f h^{\prime}+f^{\prime} h+h h^{\prime}\right) .
\end{aligned}
$$




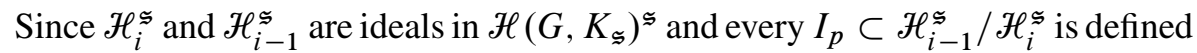
in terms of $W_{i}$-stable subvarieties of $X_{\mathrm{nr}}\left(M_{i}\right)$, this multiplication is well defined. The standard filtration of $A$ is

$$
A \supset M_{2}\left(L_{0}\right) \supset M_{2}\left(L_{0}\right) \supset M_{2}\left(L_{1}\right) \supset M_{2}\left(L_{1}\right) \supset \cdots \supset M_{2}\left(L_{\operatorname{dim} V}\right) .
$$

According to [KNS], Proposition 1 and Theorem 10, applied to the algebra $A$ and its ideal $M_{2}\left(L_{0}\right)=M_{2}\left(I_{p-1} / I_{p}\right)$, there are natural isomorphisms

$$
\operatorname{HP}_{*}\left(M_{2}\left(L_{q-1}\right) / M_{2}\left(L_{q}\right)\right) \cong H^{[*]}\left(Y_{p, q} \cup Z_{p}, Y_{p, q-1} \cup Z_{p}\right) .
$$

Let $\tilde{Y}_{p, q}$ be the inverse image of $Y_{p, q}$ under the map $\tilde{Y}_{p} \backslash \widetilde{Z}_{p} \rightarrow Y_{p} \backslash Z_{p}$ from (3.8). To (3.13) corresponds the filtration

$$
Z\left(B_{p}\right)=F_{0} \supset F_{1} \supset \cdots \supset F_{\operatorname{dim} V}=0, \quad F_{q}:=\mathcal{O}_{0}\left(\tilde{Y}_{p}, \tilde{Y}_{p, q} \cup Z_{p}\right) .
$$

By construction (3.11) induces a map

$$
\tau_{q}: \operatorname{HP}_{*}\left(L_{q-1} / L_{q}\right) \rightarrow \operatorname{HP}_{*}\left(F_{q-1} / F_{q}\right)^{w_{i}},
$$

which is natural with respect to morphisms of the underlying varieties. By Theorem 1.6.a the right-hand side is isomorphic to

$$
\begin{aligned}
& \operatorname{HP}_{*}\left(\mathcal{O}_{0}\left(\tilde{Y}_{p, q} \cup \tilde{Z}_{p}, \tilde{Y}_{p, q-1} \cup \tilde{Z}_{p}\right)\right)^{w_{i}} \\
& \cong \operatorname{HP}_{*}\left(\mathcal{O}_{0}\left(Y_{p, q} \cup Z_{p}, Y_{p, q-1} \cup Z_{p}\right)\right) \cong H^{[*]}\left(Y_{p, q} \cup Z_{p}, Y_{p, q-1} \cup Z_{p}\right) .
\end{aligned}
$$

Since $\tau_{q}$ is natural, (3.14) and (3.15) show that it is an isomorphism. Although $\tau$ and $\tau_{q}$ are not induced by algebra homomorphisms, they do come from maps of the appropriate periodic cyclic bicomplexes, which is enough to ensure that they are compatible with the connecting maps from (1.12). Hence we can use a variation on Lemma 1.3, where the role of $\operatorname{HP}_{*}(\phi)$ is played by $\tau_{*}$. This leads to the conclusion that (3.11) is an isomorphism.

We return to the proof of Proposition 3.4. Lemmas 3.5 and 3.6 allow us to write down the following diagram:

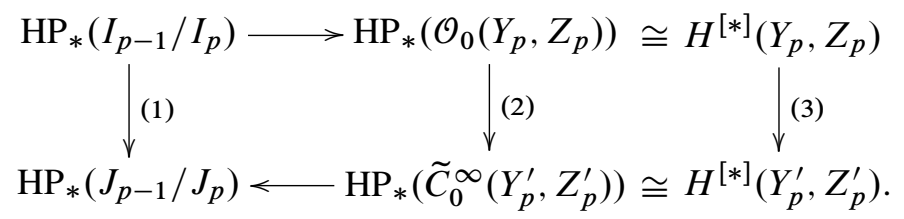

By Lemma $2.16\left(Y_{p}^{\prime}, Z_{p}^{\prime}\right)$ is a deformation retract of $\left(Y_{p}, Z_{p}\right)$, so (3) and (2) are isomorphisms. Unfortunately the diagram does not commute, because unlike (3.10) $\widetilde{C}_{0}^{\infty}\left(Y_{p}^{\prime}, Z_{p}^{\prime}\right) \rightarrow J_{p-1} / J_{p}$ is not a "rank one" inclusion. However, all the vector spaces in the diagram decompose as direct sums over the components of $Y_{p} \backslash Z_{p}$, which 
were labelled $(C, n)$ on p. 542. For every such component the diagram commutes up to a scalar factor, namely the dimension of the corresponding module $V_{n}^{x}$ from (3.7). Therefore the diagram does show that the arrow (1) is an isomorphism. This concludes the proofs of Proposition 3.4 and Theorem 3.3.

We remark that a slightly simpler version of the above proof also works for affine Hecke algebras and their Schwartz completions; see [Sol2].

3.2. Example: $\mathbf{S L}_{2}\left(\mathbb{Q}_{p}\right)$. To clarify the proof of Theorem 3.3 we show in some detail what it involves in the simplest case. This section is partly based on the related calculations in [BHP2] and [Sol3], Section 6.1.

Let $p$ be an odd prime, $\mathbb{Q}_{p}$ the field of $p$-adic numbers, $\mathbb{Z}_{p}$ the ring of $p$-adic integers and $p \mathbb{Z}_{p}$ its unique maximal ideal. We consider the reductive group $G=$ $\mathrm{SL}_{2}\left(\mathbb{Q}_{p}\right)$ with the maximal torus $A=\left\{\left(\begin{array}{cc}a & 0 \\ 0 & a^{-1}\end{array}\right) \mid a \in \mathbb{Q}_{p}^{\times}\right\}$and the minimal parabolic subgroup $P=\left\{\left(\begin{array}{cc}a & b \\ 0 & a^{-1}\end{array}\right) \mid a \in \mathbb{Q}_{p}^{\times}, b \in \mathbb{Q}_{p}\right\}$. We have

$$
\begin{aligned}
& M=Z_{G}(A)=A, \\
& W=N_{G}(A) / Z_{G}(A)=\left(A \cup\left(\begin{array}{cc}
0 & -1 \\
1 & 0
\end{array}\right) A\right) / A .
\end{aligned}
$$

The Iwahori subgroup is $K=\left\{\left(\begin{array}{ll}a & b \\ c & d\end{array}\right) \mid a, b, d \in \mathbb{Z}_{p}, c \in p \mathbb{Z}_{p}\right\}$. In this situation $\mathscr{H}(G, K)$ is Morita equivalent to $\mathscr{H}(G)^{\mathfrak{s}}$, where $\mathfrak{s}=[M, \sigma] \in \Omega(G)$ is the Borel component corresponding to the trivial representation $(\sigma, E)$ of $M$. According to [IwMa], $\mathscr{H}(G, K)$ is isomorphic to the Iwahori-Hecke algebra $\mathscr{H}\left(A_{1}, p\right)$ of type $A_{1}$ with parameter $p$. Furthermore $\delta(G, K)$ is isomorphic to the Schwartz completion $\delta\left(A_{1}, p\right)$ of $\mathscr{H}\left(A_{1}, p\right)$; see [DeOp1].

We identify $X_{\mathrm{nr}}(M)$ with $\mathbb{C}^{\times}$by evaluation at $\left(\begin{array}{cc}p & 0 \\ 0 & p^{-1}\end{array}\right)$. For almost all $\chi \in$ $X_{\mathrm{nr}}(M)$ the $G$-representation $I(P, A, \sigma, \chi)$ is irreducible, so the separated quotient of $\operatorname{Prim}\left(\mathscr{H}(G)^{\mathfrak{F}}\right.$ is

$$
X_{\mathrm{nr}}(M) / W \cong \mathbb{C}^{\times} /\left(z \sim z^{-1}\right) .
$$

The $K$-invariant part $I^{\prime}(\chi)$ of $I(P, A, \sigma, \chi)$ is a two-dimensional $\mathscr{H}(G, K)$-module with underlying vector space $V_{\chi}=V:=I_{P}^{G}(E)^{K}$. The intertwining operator $u_{w}(\chi)$ has rank one if $\chi^{2}=p^{ \pm 1}$ and is invertible for all other $\chi \in X_{\mathrm{nr}}(M)$. More precisely the homomorphism

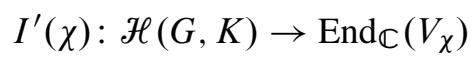

is surjective for the generic $\chi$ and has image conjugate to $\left\{\left(\begin{array}{cc}a & b \\ 0 & d\end{array}\right) \mid a, b, d \in \mathbb{C}\right\}$ for special $\chi$. Therefore Prim $(\mathscr{H}(G, K))$ has only two pairs of nonseparated points at

$$
W \chi=p^{ \pm 1 / 2} \text { and } W \chi=-p^{ \pm 1 / 2} .
$$

The $\mathscr{H}(G, K)$-modules $V_{\chi}$ with $\chi \in X_{\text {unr }}(M)$ extend continuously to $\delta(G, K)$ modules. Besides that, $S(G, K)$ admits precisely two inequivalent one-dimensional square-integrable modules, namely the irreducible submodules of $V_{p^{1 / 2}}$ and of $V_{-p^{1 / 2}}$. 
Hence Prim $(\mathcal{S}(G, K))$ consists of two isolated points (say $\delta_{+}$and $\delta_{-}$) and a copy of $X_{\text {unr }}(M) / W \cong[-1,1]$. In the filtrations

$$
\begin{aligned}
\mathscr{H}\left(G, K_{\mathfrak{S}}\right)^{\mathfrak{S}} & =\mathscr{H}_{0}^{\mathfrak{S}} \supset \mathscr{H}_{1}^{\mathfrak{s}} \supset \mathscr{H}_{2}^{\mathfrak{s}}=0, \\
\mathcal{S}\left(G, K_{\mathfrak{s}}\right)^{\mathfrak{s}} & =S_{0}^{\mathfrak{S}} \supset S_{1}^{\mathfrak{S}} \supset S_{2}^{\mathfrak{s}}=0
\end{aligned}
$$

we have

$$
\begin{aligned}
\mathcal{S}_{0}^{\mathfrak{S}} & \cong C^{\infty}\left(X_{\mathrm{nr}}(M)\right)^{W} \otimes \operatorname{End}_{\mathbb{C}}(V) \oplus \mathbb{C} \oplus \mathbb{C}, \\
\mathcal{S}_{1}^{\mathfrak{S}} & \cong C^{\infty}\left(X_{\mathrm{nr}}(M)\right)^{W} \otimes \operatorname{End}_{\mathbb{C}}(V), \\
\mathscr{H}_{1}^{\mathfrak{s}} & =\operatorname{ker}\left(\delta_{+}\right) \cap \operatorname{ker}\left(\delta_{-}\right) \subset \mathcal{O}\left(X_{\mathrm{nr}}(M) ; \operatorname{End}_{\mathbb{C}}(V)\right)^{W}, \\
\mathscr{H}_{0}^{\mathfrak{S}} / \mathscr{H}_{1}^{\mathfrak{s}} & \cong S_{0}^{\mathfrak{S}} / \mathcal{S}_{1}^{\mathfrak{S}} \cong \operatorname{End}_{\mathbb{C}}\left(\delta_{+}\right) \oplus \operatorname{End}_{\mathbb{C}}\left(\delta_{-}\right) \cong \mathbb{C} \oplus \mathbb{C} .
\end{aligned}
$$

The tricky step is to see that $\operatorname{HP}_{*}\left(\mathscr{H}_{1}^{\mathfrak{s}}\right) \cong \operatorname{HP}_{*}\left(\mathcal{S}_{1}^{\mathfrak{S}}\right)$. Clearly

$$
\begin{aligned}
\operatorname{Prim}\left(S_{1}^{\mathfrak{T}}\right) & \cong X_{\mathrm{unr}}(M) / W \\
Z\left(S_{1}^{\mathfrak{s}}\right) & \cong C^{\infty}\left(X_{\mathrm{unr}}(M)\right)^{W} \\
\operatorname{HP}_{*}\left(\left(_{1}^{\mathfrak{F}}\right)\right. & \cong \mathrm{HP}_{*}\left(Z\left(\left(_{1}^{\mathfrak{F}}\right)\right) \cong H^{[*]}\left(X_{\mathrm{unr}}(M) / W\right) \cong \check{H}^{*}([-1,1] ; \mathbb{C})\right.
\end{aligned}
$$

However the image of

$$
I^{\prime}\left( \pm p^{1 / 2}\right): \mathscr{H}_{1}^{\mathfrak{S}} \rightarrow \operatorname{End}_{\mathbb{C}}\left(V_{ \pm p^{1 / 2}}\right)
$$

is not $M_{2}(\mathbb{C})$ but is conjugate to $\left\{\left(\begin{array}{ll}0 & b \\ 0 & d\end{array}\right) \mid b, d \in \mathbb{C}\right\}$. Therefore

$$
Z\left(\mathscr{H}_{1}^{\mathfrak{F}}\right) \cong \mathcal{O}_{0}\left(X_{\mathrm{nr}}(M) / W,\left\{p^{ \pm 1 / 2},-p^{ \pm 1 / 2}\right\}\right),
$$

even though $\operatorname{Prim}\left(\mathcal{H}_{1}^{\mathfrak{s}}\right) \cong X_{\mathrm{nr}}(M) / W$. Consider the diagram

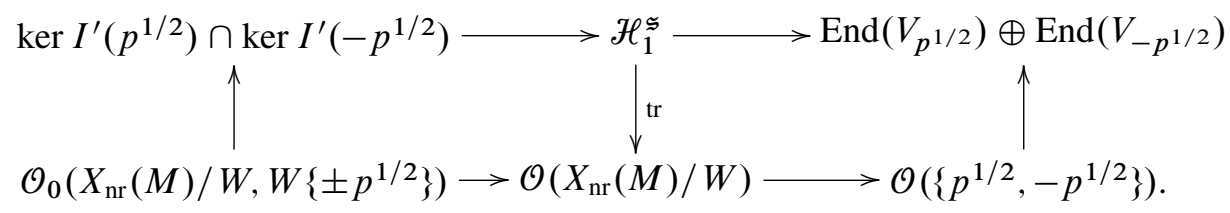

The upward arrows identify the centers of the respective algebras. These morphisms are spectrum preserving, so they induce isomorphisms on periodic cyclic homology. The downward arrow is the (generalized) trace map, which induces a map

$$
\mathrm{HP}_{*}(\operatorname{tr}): \operatorname{HP}_{*}\left(\mathscr{H}_{1}^{\mathfrak{s}}\right) \rightarrow \operatorname{HP}_{*}\left(\mathcal{O}\left(X_{\mathrm{nr}}(M)\right)\right)^{W} \cong \operatorname{HP}_{*}\left(\mathcal{O}\left(X_{\mathrm{nr}}(M) / W\right)\right) .
$$

Now we apply the functor $\mathrm{HP}_{*}$ to the entire diagram, and we replace the downward arrow by $\frac{1}{2} \mathrm{HP}_{*}(\mathrm{tr})$. The resulting diagram commutes and shows that $\mathrm{HP}_{*}(\mathrm{tr})$ is a 
natural isomorphism. From the commutative diagram

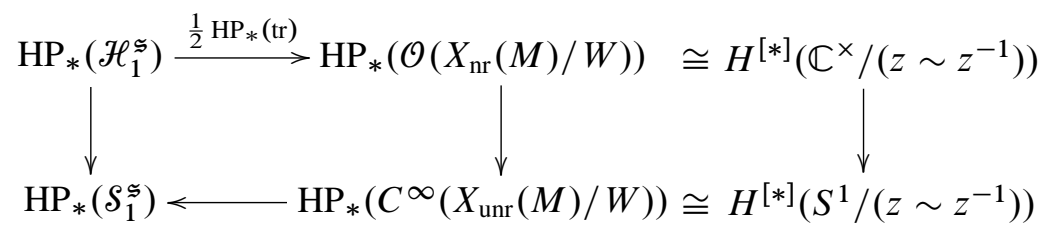

we see that the left vertical arrow is indeed an isomorphism. From this we can derive a natural isomorphism

$$
\operatorname{HP}_{*}\left(\mathscr{H}(G)^{\mathfrak{s}}\right) \cong \operatorname{HP}_{*}(\mathscr{H}(G, K)) \rightarrow \operatorname{HP}_{*}(S(G, K), \hat{\otimes}) \cong \operatorname{HP}_{*}\left(S(G)^{\mathfrak{s}}, \hat{\otimes}_{\mathbb{C}}\right) .
$$

We remark that the Borel component is the most complicated Bernstein component of $\mathrm{SL}_{2}\left(\mathbb{Q}_{p}\right)$. Indeed with one exception every other $\mathfrak{s} \in \Omega\left(\mathrm{SL}_{2}\left(\mathbb{Q}_{p}\right)\right)$ has a trivial Weyl group, and therefore $\operatorname{Prim}\left(\mathscr{H}\left(\mathrm{SL}_{2}\left(\mathbb{Q}_{p}\right)\right)^{\mathfrak{s}}\right)$ is homeomorphic to either $\mathbb{C}^{\times}$or a point. Moreover both $\mathscr{H}\left(\mathrm{SL}_{2}\left(\mathbb{Q}_{p}\right)\right)^{\mathfrak{F}}$ and $S\left(\mathrm{SL}_{2}\left(\mathbb{Q}_{p}\right)\right)^{\mathfrak{s}}$ are Morita equivalent to commutative algebras for such $\mathfrak{s}$.

3.3. Equivariant cosheaf homology. The most interesting applications of the results of Section 3.1 lie in their connection with the Baum-Connes conjecture. To make this relation precise we need several additional homology theories. In particular, our forthcoming discussing will require some detailed knowledge of equivariant cosheaf homology. Therefore we first provide an overview of this theory, which is mostly taken from [BCH], [HiNi].

Let $G$ be a totally disconnected group and $\Sigma$ a polysimplicial complex. We assume that $\Sigma$ is equipped with a polysimplicial $G$-action, which is proper in the sense that the isotropy group $G_{\sigma}$ of any polysimplex $\sigma$ is compact and open. Let $\Sigma^{p}$ denote the collection of $p$-dimensional polysimplices of $\Sigma$, endowed with the discrete topology. Define the vector space

$$
C_{p}(G ; \Sigma):=\bigoplus_{\sigma \in \Sigma^{p}} C_{c}^{\infty}\left(G_{\sigma}\right)
$$

where $C^{\infty}(X)$ denotes the set of locally constant complex valued functions on a totally disconnected space $X$. We write the elements of $C_{p}(G ; \Sigma)$ as formal sums $\sum_{\sigma} f_{\sigma}[\sigma]$. If $\tau$ is a face of $\sigma$ then $G_{\tau} \supset G_{\sigma}$, so we may consider $f_{\sigma}$ as a locally constant function on $G_{\tau}$. On every polysimplex we fix an orientation and identify $[\bar{\sigma}]$ with $-[\sigma]$, where $\bar{\sigma}$ means $\sigma$ with the opposite orientation. We write the simplicial boundary operator as

$$
\delta \sigma=\sum_{\tau \in \Sigma^{p-1}}[\sigma: \tau] \tau \quad \text { with }[\sigma: \tau] \in\{-1,0,1\} .
$$

This gives a differential

$$
\delta_{p}: C_{p}(G ; \Sigma) \rightarrow C_{p-1}(G ; \Sigma), \quad \delta_{p}\left(f_{\sigma}[\sigma]\right)=\sum_{\tau \in \Sigma^{p-1}}[\sigma: \tau] f_{\sigma}[\tau] .
$$


We endow the differential complex $\left(C_{*}(G ; \Sigma), \delta_{*}\right)$ with the $G$-action

$$
g \cdot f_{\sigma}[\sigma]=f_{\sigma}^{g}[g \sigma],
$$

where $f_{\sigma}^{g} \in C_{c}^{\infty}\left(G_{g \sigma}\right)$ is defined by $f_{\sigma}^{g}(h)=f_{\sigma}\left(g^{-1} h g\right)$ and $g \sigma$ is endowed with the orientation coming from our chosen orientation on $\sigma$. Clearly $\delta_{*}$ is $G$ equivariant, so it is well defined on the space $C_{*}(G ; \Sigma)_{G}$ of $G$-coinvariants. The equivariant cosheaf homology of $\Sigma$ is

$$
\mathrm{CH}_{n}^{G}(\Sigma):=H_{n}\left(C_{*}(G ; \Sigma)_{G}, \delta_{*}\right) .
$$

There is also a relative version of this theory. Let $\Sigma^{\prime}$ be a $G$-stable subcomplex of $\Sigma$. We define the relative equivariant cosheaf homology of $\left(\Sigma, \Sigma^{\prime}\right)$ as

$$
\mathrm{CH}_{n}^{G}\left(\Sigma, \Sigma^{\prime}\right):=H_{n}\left(C_{*}(G ; \Sigma)_{G} / C_{*}\left(G ; \Sigma^{\prime}\right)_{G}, \delta_{*}\right) .
$$

As usual there is a long exact sequence in homology:

$$
\cdots \rightarrow \mathrm{CH}_{n+1}^{G}\left(\Sigma, \Sigma^{\prime}\right) \rightarrow \mathrm{CH}_{n}^{G}\left(\Sigma^{\prime}\right) \rightarrow \mathrm{CH}_{n}^{G}(\Sigma) \rightarrow \mathrm{CH}_{n}^{G}\left(\Sigma, \Sigma^{\prime}\right) \rightarrow \cdots .
$$

If $G$ acts freely on $\Sigma$ then $\mathrm{CH}_{n}^{G}\left(\Sigma, \Sigma^{\prime}\right)$ reduces to the usual simplicial homology $H_{n}\left(\Sigma / G, \Sigma^{\prime} / G\right)$ with complex coefficients.

Higson and Nistor [HiNi] introduced a natural map

$$
\mathrm{CH}_{n}(\Sigma) \rightarrow \mathrm{HH}_{n}\left(C_{c}^{\infty}(G)\right),
$$

whose construction we recall in as much detail as we need. Let

$$
{ }_{n} \hat{\Sigma}^{p}:=\left\{\left(g_{0}, g_{1}, \ldots, g_{n}, \sigma\right) \in G^{n+1} \times \Sigma^{p} \mid g_{0} g_{1} \ldots g_{n} \sigma=\sigma\right\}
$$

be the $n$th Brylinski space of $\Sigma^{p}$. By definition $\Sigma^{p}$ is discrete, so ${ }_{n} \hat{\Sigma}^{p}$ is a totally disconnected space and $C_{c}^{\infty}\left({ }_{n} \hat{\Sigma}^{p}\right)$ is defined. According to [HiNi], Section 4, there is an exact sequence

$$
0 \leftarrow C_{p}(G ; \Sigma)_{G} \leftarrow C_{c}^{\infty}\left({ }_{0} \hat{\Sigma}^{p}\right) \leftarrow C_{c}^{\infty}\left({ }_{1} \hat{\Sigma}^{p}\right) \leftarrow C_{c}^{\infty}\left({ }_{2} \hat{\Sigma}^{p}\right) \leftarrow \cdots .
$$

Consequently $\mathrm{CH}_{*}^{G}(\Sigma)$ can be computed as the homology of a double complex

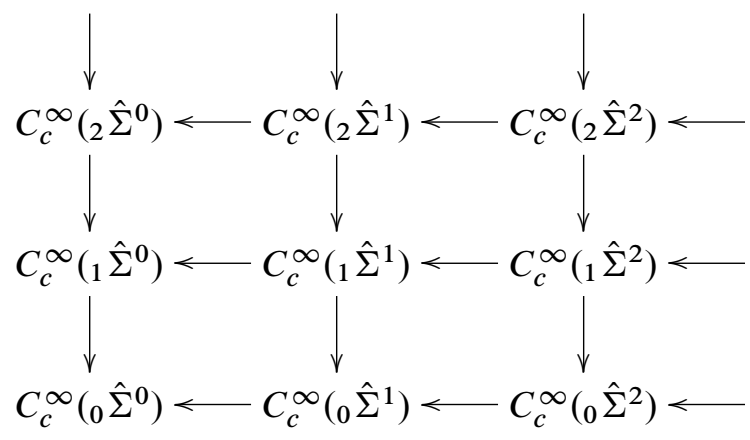


In this diagram the horizontal maps come from the boundary map $\partial$ on $\Sigma$, while the vertical maps are essentially the differentials for a Hochschild complex. There are natural maps

$$
C_{c}^{\infty}\left({ }_{n} \hat{\Sigma}^{0}\right) \rightarrow C_{c}^{\infty}\left(G^{n+1}\right), f \rightarrow\left(\left(g_{0}, \ldots, g_{n}\right) \mapsto \sum_{x \in \Sigma^{0}} f\left(g_{0}, \ldots, g_{n}, x\right)\right)
$$

from the double complex (3.22) to the standard Hochschild complex for $C_{c}^{\infty}(G)$. Together (3.21), (3.22) and (3.23) yield the map (3.20).

Suppose now that $G$ acts properly on some affine building. The Hochschild homology of $C_{c}^{\infty}(G)$ admits a decomposition

$$
\mathrm{HH}_{n}\left(C_{c}^{\infty}(G)\right)=\mathrm{HH}_{n}\left(C_{c}^{\infty}(G)\right)_{\mathrm{ell}} \oplus \mathrm{HH}_{n}\left(C_{c}^{\infty}(G)\right)_{\text {hyp }}
$$

into an elliptic and an hyperbolic part. Upon periodization the hyperbolic part disappears and one finds [HiNi], Section 7,

$$
\operatorname{HP}_{n}\left(C_{c}^{\infty}(G)\right)=\bigoplus_{m \in \mathbb{Z}} \mathrm{HH}_{n+2 m}\left(C_{c}^{\infty}(G)\right)_{\mathrm{ell}} .
$$

This and (3.20) yield a map

$$
\mu_{\mathrm{HN}}: \mathrm{CH}_{[*]}^{G}(\Sigma) \rightarrow \mathrm{HP}_{*}\left(C_{c}^{\infty}(G)\right) .
$$

As Higson and Nistor observe, this is an algebraic analogue of the Baum-Connes assembly map. Let $h: X \rightarrow \Sigma$ be any morphism of proper $G$-simplicial complexes. It induces a map between the corresponding bicomplexes (3.22), and from the explicit formula (3.23) we see that $\mu_{\mathrm{HN}}$ for $X$ factors as

$$
\mathrm{CH}_{n}^{G}(X) \stackrel{\mathrm{CH}_{n}^{G}(h)}{\longrightarrow} \mathrm{CH}_{n}^{G}(\Sigma) \rightarrow \mathrm{HH}_{n}\left(C_{c}^{\infty}(G)\right) \rightarrow \mathrm{HP}_{n}\left(C_{c}^{\infty}(G)\right) .
$$

Higson and Nistor [HiNi] showed that (3.25) is an isomorphism if $\Sigma$ is an affine building. The special case where $G$ is a semisimple $p$-adic group was also proved by Schneider [Sch].

3.4. The Baum-Connes conjecture. Let $G$ be any locally compact group acting properly on a Hausdorff space $\Sigma$. A subspace $X \subset \Sigma$ is called $G$-compact if $X / G$ is compact. The equivariant $K$-homology of $\Sigma$ is defined as

$$
K_{*}^{G}(\Sigma)=\underline{\lim } \mathrm{KK}_{*}^{G}\left(C_{0}(X), \mathbb{C}\right),
$$

where $\mathrm{KK}_{*}^{G}$ is Kasparov's equivariant KK-theory [Kas] and the limit runs over all $G$ compact subspaces $X$ of $\Sigma$. The Baum-Connes conjecture asserts that the assembly map

$$
\mu: K_{*}^{G}(\Sigma) \rightarrow K_{*}\left(C_{r}^{*}(G)\right)
$$


is an isomorphism if $\Sigma$ is a classifying space for proper $G$-actions. Building upon the work of Kasparov, Vincent Lafforgue proved this conjecture for many groups, including all locally compact groups that act properly isometrically on an affine building [Laf].

Now we specialize to a reductive $p$-adic group $G$. In this case the affine BruhatTits building $\beta G$ is a classifying space for proper $G$-actions. We recall that $\beta G$ is a finite dimensional locally finite polysimplicial complex endowed with an isometric $G$-action such that $\beta G / G$ is compact and contractible. For any $X$ as above there exists a continuous $G$-map $h: X \rightarrow \beta G$, and it is unique up to homotopy. The assembly map $\mu: K_{*}^{G}(X) \rightarrow K_{*}\left(C_{r}^{*}(G)\right.$ factors as

$$
K_{*}^{G}(X) \stackrel{K_{*}^{G}(h)}{\longrightarrow} K_{*}^{G}(\beta G) \stackrel{\mu}{\rightarrow} K_{*}\left(C_{r}^{*}(G)\right) .
$$

A natural receptacle for a Chern character from $K_{*}^{G}(X)$ is formed by

$$
\mathrm{HL}_{*}^{G}(X):=\mathrm{HL}_{*}^{G}\left(C_{0}(X), \mathbb{C}\right),
$$

where $\mathrm{HL}_{*}^{G}$ denotes equivariant local cyclic homology, as defined and studied by Voigt [Voi2]. With these notions we can state and prove a more precise version of [BHP3], Proposition 9.4. We note that a similar idea was already used in [BHP2] to prove the Baum-Connes conjecture for $G=\mathrm{GL}_{n}(\mathbb{F})$.

Theorem 3.7. There exists a commutative diagram

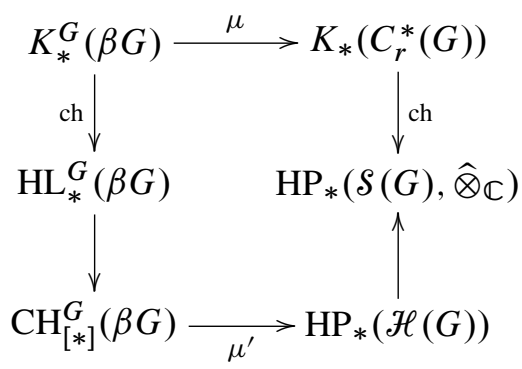

with the following properties:

a) Both Chern characters become isomorphisms after applying $\otimes_{\mathbb{Z}} \mathbb{C}$ to their domain.

b) The other maps are natural isomorphisms.

Proof. As mentioned before, Lafforgue [Laf] showed that the assembly map $\mu$ is an isomorphism. The right column is taken care of by Theorems 3.2 and 3.3.

Let $\Sigma$ be a finite dimensional, locally finite $G$-compact $G$-simplicial complex. It was proved in [Voi2], Proposition 10.4, that the inclusion map $C_{c}^{\infty}(\Sigma) \rightarrow C_{0}(\Sigma)$ induces an isomorphism

$$
\mathrm{HL}_{*}^{G}(\Sigma)=\mathrm{HL}_{*}^{G}\left(C_{0}(\Sigma), \mathbb{C}\right) \rightarrow \mathrm{HL}_{*}^{G}\left(C_{c}^{\infty}(\Sigma), \mathbb{C}\right) .
$$


Let $\mathrm{HP}_{*}^{G}$ denote Voigt's equivariant periodic cyclic homology [Voi1], Section 3. According to [Voi3], Section 6, there are natural isomorphisms

$$
\begin{aligned}
& \mathrm{HL}_{*}^{G}\left(C_{c}^{\infty}(\Sigma), \mathbb{C}\right) \cong \mathrm{HP}_{*}^{G}\left(C_{c}^{\infty}(\Sigma), \mathbb{C}\right), \\
& \text { ch: } \mathrm{KK}_{*}^{G}\left(C_{0}(\Sigma), \mathbb{C}\right) \otimes_{\mathbb{Z}} \mathbb{C} \rightarrow \mathrm{HL}_{*}^{G}\left(C_{0}(\Sigma), \mathbb{C}\right) \text {. }
\end{aligned}
$$

We have to check that

$$
\mathrm{HP}_{*}^{G}\left(C_{c}^{\infty}(\Sigma), \mathbb{C}\right) \cong \mathrm{CH}_{[*]}^{G}(\Sigma)
$$

Baum and Schneider $[\mathrm{BaSc}]$, Section 1.B, showed that cosheaf homology can be regarded as a special case of a bivariant (co)homology theory:

$$
\mathrm{CH}_{n}^{G}(\Sigma) \cong H_{G}^{n}(\Sigma \text {, point }) \text {. }
$$

According to [Voi1] the right-hand side of (3.31) is naturally isomorphic to the lefthand side of (3.30), so we get natural isomorphisms

$$
K_{*}^{G}(\Sigma) \otimes_{\mathbb{Z}} \mathbb{C} \rightarrow \mathrm{HL}_{*}^{G}(\Sigma) \rightarrow \mathrm{CH}_{[*]}^{G}(\Sigma) .
$$

The case $\Sigma=\beta G$ gives us the left column of the theorem. To complete the proof we define

$$
\mu^{\prime}: \mathrm{CH}_{[*]}^{G}(\beta G) \rightarrow \mathrm{HP}_{*}(\mathscr{H}(G))
$$

as the unique map so that the diagram commutes.

It is not immediately clear that $\mu^{\prime}$ from (3.33) is the same map as $\mu_{\mathrm{HN}}$ from (3.25), for $C_{c}^{\infty}(G)=\mathscr{H}(G)$ and $\Sigma=\beta G$. We will prove this by reduction to the following simpler case. Let $U \in \operatorname{CO}(G)$ and consider the discrete proper homogeneous $G$ space $G / U$. By the universal property of $\beta G$ there exists a continuous $G$-equivariant map $G / U \rightarrow \beta G$, and it is unique up to homotopy. With a suitable simplicial subdivision of $\beta G$ we can achieve that this is in fact a simplicial $G$-map.

Lemma 3.8. The following diagram commutes for elements in the upper left corner.

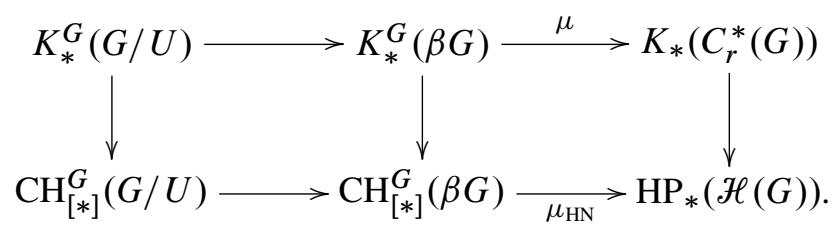

Proof. The left-hand square commutes by functoriality. It follows readily from the definitions that $K_{*}^{G}(G / U) \cong K_{*}^{U}$ (point). By the functoriality of the Baum-Connes assembly map there is a commutative diagram

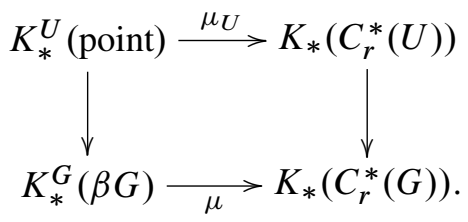


Since $U$ is compact and totally disconnected, both $K_{*}^{U}$ (point) and $K_{*}\left(C_{r}^{*}(U)\right)$ are naturally isomorphic to the ring of smooth (virtual) representations $R(U)$, and $\mu_{U}$ corresponds to the composition of these isomorphisms. The right vertical map comes from the inclusion $C_{r}^{*}(U) \rightarrow C_{r}^{*}(G)$, so it sends a $U$-module $V$ to $\operatorname{Ind}_{U}^{G}(V)$.

Similarly there are a canonical isomorphism

$$
\mathrm{CH}_{*}^{G}(G / U) \cong \mathrm{CH}_{*}^{U} \text { (point) }
$$

and a commutative diagram

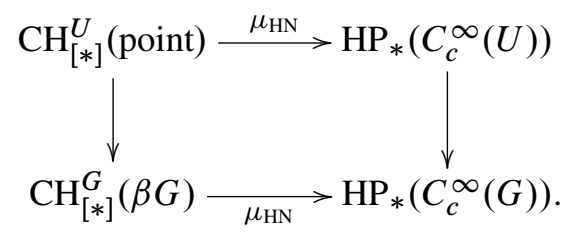

According to [HiNi], Section 4, we have $\operatorname{HP}_{1}\left(C_{c}^{\infty}(U)\right)=0$ and

$$
\mathrm{HP}_{0}\left(C_{c}^{\infty}(U)\right)=\mathrm{HH}_{0}\left(C_{c}^{\infty}(U)\right)=C_{c}^{\infty}(U)_{U} .
$$

By definition, also

$$
\mathrm{CH}_{n}^{U} \text { (point) }= \begin{cases}C_{c}^{\infty}(U)_{U} & \text { if } n=0, \\ 0 & \text { if } n>0 .\end{cases}
$$

A glance at the double complex (3.22) shows that

$$
\mu_{\mathrm{HN}}: \mathrm{CH}_{0}^{U} \text { (point) } \rightarrow \mathrm{HP}_{0}\left(C_{c}^{\infty}(U)\right)
$$

corresponds to the identity map under these identifications. Furthermore $U$ is profinite, so

$$
C_{c}^{\infty}(U)=\lim _{\longrightarrow} \mathbb{C}[F]
$$

where the limit runs over all finite quotient groups $F$ of $U$. Similarly we can write $C_{r}^{*}(U)$ as an inductive limit in the category of $C^{*}$-algebras. In this situation both $K_{*}$ and $\mathrm{HP}_{*}$ commute with $\stackrel{\lim }{\longrightarrow}$, so we get a Chern character

$$
K_{*}\left(C_{r}^{*}(U)\right) \cong \lim _{\longrightarrow} K_{*}(\mathbb{C}[F]) \rightarrow \underset{\lim }{\longrightarrow} \mathrm{HP}_{*}(\mathbb{C}[F]) \cong \mathrm{HP}_{*}\left(C_{c}^{\infty}(U)\right) .
$$

Now the right-hand square of the diagram

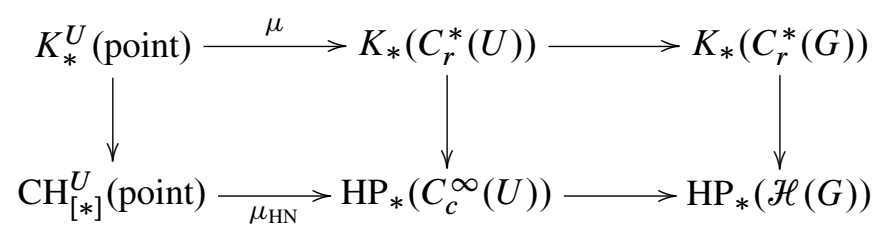


commutes by functoriality. According to Voigt [Voi2], Proposition 13.5, the Chern character

$$
K_{*}^{U} \text { (point) } \rightarrow \mathrm{HL}_{*}^{U} \text { (point) }
$$

can be identified with the character map $R(U) \rightarrow C_{c}^{\infty}(U)^{U}$. The isomorphism between $\mathrm{HL}_{*}^{U}$ (point) and $\mathrm{CH}_{*}^{U}$ (point) then becomes the canonical map

$$
C_{c}^{\infty}(U)^{U} \rightarrow C_{c}^{\infty}(U)_{U}
$$

which is bijective because $U$ is compact. Since the Chern character for $\mathbb{C}[F]$ in (3.36) may also be identified with the character map, we find that the left-hand square of (3.37) commutes. Together the commutative diagrams (3.34), (3.35) and (3.37) complete the proof.

Lemma 3.9. The maps $\mu_{\mathrm{HN}}$ from (3.25) and $\mu^{\prime}$ from (3.33) are the same.

Proof. We have to show that the diagram

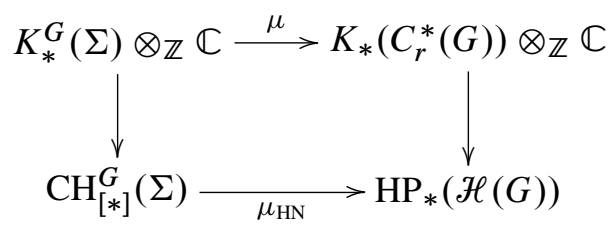

commutes for $\Sigma=\beta G$. By subdividing all polysimplices we may assume that $\beta G$ is a simplicial complex, and that $G$ preserves this structure. Let $\beta^{(n)} G$ denote the $n$-skeleton of $\beta G$, and $(\beta G)^{n}$ the collection of $n$-simplices. Both inherit a $G$-action from $\beta G$. We will prove the commutativity of (3.38) for $\Sigma=\beta^{(n)}$, with induction to $n$.

The set $\beta^{(0)} G$ is a finite union of $G$-spaces of the form $G / U$ with $U \in \operatorname{CO}(G)$, so the case $n=0$ follows from Lemma 3.8. Similarly (3.38) commutes for $\Sigma=(\beta G)^{n}$.

We consider $(\beta G)^{n} \times S^{n}$ as a $G$-space with a trivial action on $S^{n}$. The long exact sequence (3.19) for the pair $\left((\beta G)^{n} \times S^{n},(\beta G)^{n} \times\right.$ point) reads

$$
\begin{aligned}
\cdots & \rightarrow \mathrm{CH}_{p}^{G}\left((\beta G)^{n}\right) \rightarrow \mathrm{CH}_{p}^{G}\left((\beta G)^{n} \times S^{n}\right) \stackrel{\phi}{\rightarrow} \mathrm{CH}_{p-n}^{G}\left((\beta G)^{n}\right) \otimes \mathbb{C} H_{n}\left(S^{n}, \text { point }\right) \\
& \rightarrow \mathrm{CH}_{p-1}^{G}\left((\beta G)^{n}\right) \rightarrow \mathrm{CH}_{p-1}^{G}\left((\beta G)^{n} \times S^{n}\right) \rightarrow \cdots .
\end{aligned}
$$

We note that

$$
\mathrm{CH}_{p}^{G}\left((\beta G)^{n} \times S^{n}\right) \cong \mathrm{CH}_{p}^{G}\left((\beta G)^{n}\right) \otimes_{\mathbb{C}} H_{0}\left(S^{n}\right) \oplus \mathrm{CH}_{p-n}^{G}\left((\beta G)^{n}\right) \otimes_{\mathbb{C}} H_{n}\left(S^{n}, \text { point }\right),
$$

so $\phi$ is surjective and the sequence splits. Since $\mu_{\mathrm{HN}}-\mu^{\prime}=0$ on $\mathrm{CH}_{*}^{G}\left((\beta G)^{n}\right)$, 
there exists a unique map $f$ making the following diagram commutative:

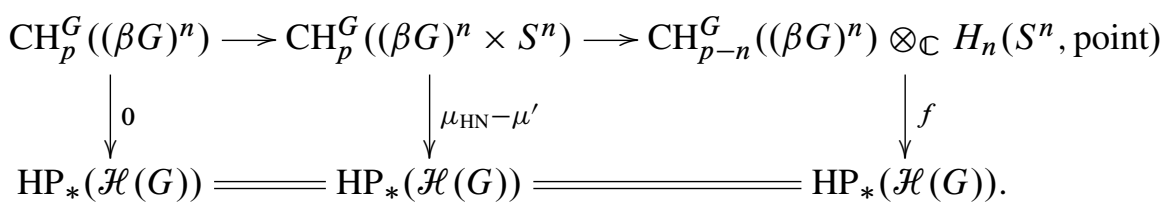

By the universal property of $\beta G$, there exists a $G$-map $h:(\beta G)^{n} \times S^{n} \rightarrow \beta G$, and it is unique up to homotopy. Hence we may assume that $h$ maps $\{\sigma\} \times S^{n} \subset(\beta G)^{n} \times S^{n}$ to the barycenter of $\sigma$ in $\beta G$. By (3.26) and (3.29) the middle map in (3.39) factors as

$$
\mathrm{CH}_{p}^{G}\left((\beta G)^{n} \times S^{n}\right) \stackrel{\mathrm{CH}_{p}^{G}(h)}{\longrightarrow} \mathrm{CH}_{p}^{G}(\beta G) \stackrel{\mu_{\mathrm{HN}}-\mu^{\prime}}{\longrightarrow} \mathrm{HP}_{*}(\mathscr{H}(G)) .
$$

But $\mathrm{CH}_{p}^{G}(h)$ kills the $n$th homology of $S^{n}$, which in combination with (3.39) shows that (3.40) is zero. Therefore the above map $f$ must also be zero. As $G$-spaces we have

$$
\beta^{(n)} G \backslash \beta^{(n-1)} G \cong(\beta G)^{n} \times S^{n} \backslash \text { point, }
$$

with $G$ acting trivially on the last factor. The corresponding long exact sequence in equivariant cosheaf homology is

$$
\begin{aligned}
\cdots & \rightarrow \mathrm{CH}_{p}^{G}\left(\beta^{(n-1)} G\right) \rightarrow \mathrm{CH}_{p}^{G}\left(\beta^{(n)} G\right) \rightarrow \mathrm{CH}_{p-n}^{G}\left((\beta G)^{n}\right) \otimes_{\mathbb{C}} H_{n}\left(S^{n}, \text { point }\right) \\
& \rightarrow \mathrm{CH}_{p-1}^{G}\left(\beta^{(n-1)} G\right) \rightarrow \mathrm{CH}_{p-1}^{G}\left(\beta^{(n)} G\right) \rightarrow \cdots .
\end{aligned}
$$

By the induction hypothesis $\mu_{\mathrm{HN}}-\mu^{\prime}=0$ on $\mathrm{CH}_{*}^{G}\left(\beta^{(n-1)} G\right)$, so we can write down a commutative diagram

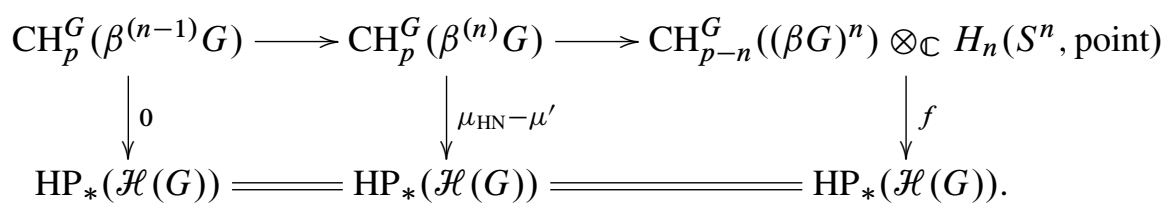

We already showed that $f=0$, so $\mu_{\mathrm{HN}}-\mu^{\prime}=0$ on $\mathrm{CH}_{p}^{G}\left(\beta^{(n)} G\right)$. Thus (3.38) commutes for $\Sigma=\beta^{(n)} G$, which completes our induction step.

The above proof can be compared with [Mey1], Section 5.1.

Corollary 3.10. It can be proved with periodic cyclic homology that the BaumConnes assembly map

$$
\mu \otimes \mathrm{id}: K_{*}^{G}(\beta G) \otimes_{\mathbb{Z}} \mathbb{Q} \rightarrow K_{*}\left(C_{r}^{*}(G)\right) \otimes_{\mathbb{Z}} \mathbb{Q}
$$

is an isomorphism for every reductive p-adic group $G$. 
Proof. Lemma 3.9 and (3.25) show that $\mu^{\prime}=\mu_{\mathrm{HN}}$ is an isomorphism. Hence all the isomorphisms in the diagram of Theorem 3.7 admit mutually independent proofs. With the commutativity of the diagram we can use any five of them to prove the sixth. In particular we can show without using Lafforgue's work that

$$
K_{*}^{G}(\beta G) \otimes_{\mathbb{Z}} \mathbb{C} \cong K_{*}\left(C_{r}^{*}(G)\right) \otimes_{\mathbb{Z}} \mathbb{C},
$$

which is equivalent to $\mu$ being a rational isomorphism.

Acknowledgements. This article is partly based on the author's PhD-thesis, which was written at the Universiteit van Amsterdam under the supervision of Eric Opdam. The author is grateful for the support and advice that Professor Opdam has given him during his $\mathrm{PhD}$ research. He would also like to thank Ralf Meyer, Christian Voigt and the referee for their comments, which lead to substantial clarifications of some proofs.

\section{References}

[ABP] A.-M. Aubert, P.F. Baum, R.J. Plymen, The Hecke algebra of a reductive $p$ adic group: a view from noncommutative geometry. In Noncommutative geometry and number theory, Aspects Math. E37, Vieweg Verlag, Wiesbaden 2006, 1-34. Zbl 1120.14001 MR 2327297

$[\mathrm{BCH}]$ P. Baum, A. Connes, and N. Higson, Classifying space for proper actions and $K$-theory of group $C^{*}$-algebras. In $C^{*}$-algebras: 1943-1993 (San Antonio, TX, 1993), Contemp. Math. 167, Amer. Math. Soc., Providence, RI, 1994, 240-291. Zbl 0830.46061 MR 1292018

[BHP1] P. Baum, N. Higson, and R. Plymen, Equivariant homology for SL(2) of a $p$-adic field. In Index theory and operator algebras (Boulder, CO, 1991), Contemp. Math. 148, Amer. Math. Soc., Providence, RI, 1993, 1-18. Zbl 0844.46043 MR 1228497

[BHP2] P. Baum, N. Higson, and R. Plymen, A proof of the Baum-Connes conjecture for $p$ adic GL(n). C. R. Acad. Sci. Paris Sér. I Math. 325 (1997), 171-176. Zbl 0918.46061 MR 1467072

[BHP3] P. Baum, N. Higson, and R. Plymen, Representation theory of $p$-adic groups: a view from operator algebras. In The mathematical legacy of Harish-Chandra (Baltimore, MD, 1998), Proc. Sympos. Pure Math. 68, Amer. Math. Soc., Providence, RI, 2000, 111-149. Zbl 0982.19006 MR 1767895

[BaNi] P. Baum and V. Nistor, Periodic cyclic homology of Iwahori-Hecke algebras. $K$ Theory 27 (2002), 329-357. Zbl 1056.16005 MR 1962907

[BaSc] P. Baum and P. Schneider, Equivariant-bivariant Chern character for profinite groups. K-Theory 25 (2002), 313-353. Zbl 0997.55010 MR 1914452

[BeDe] J. N. Bernstein and P. Deligne, Le "centre" de Bernstein. In Representations of reductive groups over a local field, Travaux en Cours, Hermann, Paris 1984, 1-32. Zbl 0599.22016 MR 0771671 
[BiSc] E. Bierstone and G. W. Schwarz, Continuous linear division and extension of $\mathcal{C}^{\infty}$ functions. Duke Math. J. 50 (1983), 233-271. Zbl 0521.32008 MR 700140

[BoWa] A. Borel and N. R. Wallach, Continuous cohomology, discrete subgroups, and representations of reductive groups. Ann. of Math. Stud. 94, Princeton University Press, Princeton, N.J., 1980. Zbl 0443.22010 MR 0554917

[Bost] J.-B. Bost, Principe d'Oka, $K$-théorie et systèmes dynamiques non commutatifs. Invent. Math. 101 (1990), 261-333. Zbl 0719.46038 MR 1062964

[BLT] J.-P. Brasselet, A. Legrand, and N. Teleman, Hochschild homology of singular algebras. K-Theory 29 (2003), 1-25. Zbl 1045.58004 MR 2024012

[BrPl] J. Brodzki and R. Plymen, Periodic cyclic homology of certain nuclear algebras. $C$. R. Acad. Sci. Paris Sér. I Math. 329 (1999), 671-676. Zbl 0955.46041 MR 1724090

[BuKu] C. J. Bushnell and P. C. Kutzko, Smooth representations of reductive $p$-adic groups: structure theory via types. Proc. London Math. Soc. (3) 77 (1998), 582-634. Zbl 0911.22014 MR 1643417

[Car] P. Cartier, Representations of $p$-adic groups: a survey. In Automorphic forms, representations and L-functions (Proc. Sympos. Pure Math., Oregon State Univ., Corvallis, Ore., 1977), Part 1, Proc. Sympos. Pure Math. 33, Amer. Math. Soc., Providence, R.I., 1979, 111-155. Zbl 0421.22010 MR 0546593

[Cas] W. Casselman, Introduction to the theory of admissible representations of $p$-adic reductive groups. Preprint 1995.

[Con] A. Connes, Non-commutative differential geometry. Inst. Hautes Études Sci. Publ. Math. 62 (1985), 41-144. Zbl 0592.46056 MR 823176

[Cun] J. Cuntz, Excision in periodic cyclic theory for topological algebras. In Cyclic cohomology and noncommutative geometry (Waterloo, ON, 1995), Fields Inst. Commun. 17, Amer. Math. Soc., Providence, RI 1997, 43-53. Zbl 0890.18006 MR 1478700

[CuQu] J. Cuntz and D. Quillen, Excision in bivariant periodic cyclic cohomology. Invent. Math. 127 (1997), 67-98. Zbl 0889.46054 MR 1423026

[DeOp1] P. Delorme and E. M. Opdam, The Schwartz algebra of an affine Hecke algebra. $J$. Reine Angew. Math. 625 (2008), 59-114. Zbl 05505173 MR 2482216

[DeOp2] P. Delorme and E. M. Opdam, Analytic $R$-groups of affine Hecke algebras. Preprint 2005.

[HC1] Harish-Chandra, Harmonic analysis on reductive $p$-adic groups. In Harmonic analysis on homogeneous spaces (Williams Coll., Williamstown, Mass., 1972), Proc. Sympos. Pure Math. 26, Amer. Math. Soc., Providence, R.I., 1973, 167-192. Zbl 0289.22018 MR 0340486

[HC2] Harish-Chandra, The Plancherel formula for reductive $p$-adic groups. In Collected papers, Vol. IV, Springer-Verlag, New York 1984, 353-367.

[HiNi] N. Higson and V. Nistor, Cyclic homology of totally disconnected groups acting on buildings. J. Funct. Anal. 141 (1996), 466-495. Zbl 0921.46083 MR 1418516

[Hum] J.E. Humphreys, Reflection groups and Coxeter groups. Cambridge Stud. Adv. Math. 29, Cambridge University Press, Cambridge 1990. Zbl 0725.20028 MR 1066460 
[Ill] S. Illman, Smooth equivariant triangulations of $G$-manifolds for $G$ a finite group. Math. Ann. 233 (1978), 199-220. Zbl 0359.57001 MR 0500993

[IwMa] N. Iwahori and H. Matsumoto, On some Bruhat decomposition and the structure of the Hecke rings of p-adic Chevalley groups. Inst. Hautes Études Sci. Publ. Math. 25 (1965), 5-48. Zbl 0228.20015 MR 0185016

[Kas] G. G. Kasparov, Equivariant $K K$-theory and the Novikov conjecture. Invent. Math. 91 (1988), 147-201. Zbl 0647.46053 MR 918241

[KNS] D. Kazhdan, V. Nistor, and P. Schneider, Hochschild and cyclic homology of finite type algebras. Selecta Math. (N.S.) 4 (1998), 321-359. Zbl 0914.16005 MR 1669952

[KnVo] A. W. Knapp and D. A. Vogan, Jr., Cohomological induction and unitary representations. Princeton Math. Ser. 45, Princeton University Press, Princeton, NJ, 1995. Zbl 0863.22011 MR 1330919

[Kon] T. Konno, A note on the Langlands classification and irreducibility of induced representations of $p$-adic groups. Kyushu J. Math. 57 (2003), 383-409. Zbl 1054.22018 MR 2050093

[Lod] J.-L. Loday, Cyclic homology. 2nd ed., Grundlehren Math. Wiss. 301, SpringerVerlag, Berlin 1998. Zbl 0885.18007 MR 1600246

[Laf] V. Lafforgue, $K$-théorie bivariante pour les algèbres de Banach et conjecture de Baum-Connes. Invent. Math. 149 (2002), 1-95. Zbl 1084.19003 MR 1914617

[Mey1] M. Meyer, Chern characters for topological groups. PhD Thesis, Universität Münster, Münster 2006.

[Mey2] R. Meyer, Homological algebra for Schwartz algebras of reductive $p$-adic groups. In Noncommutative geometry and number theory, Aspects Math. E37, Vieweg Verlag, Wiesbaden 2006, 263-300. Zbl 1111.22016 MR 2327309

[Mey3] R. Meyer, Local and analytic cyclic homology, EMS Tracts Math. 3, EMS Publishing House, Zürich 2007. Zbl 1134.46001 MR 2337277

[Nis] V. Nistor, A non-commutative geometry approach to the representation theory of reductive $p$-adic groups: homology of Hecke algebras, a survey and some new results. In Noncommutative geometry and number theory, Aspects Math. E37, Vieweg Verlag, Wiesbaden 2006, 301-321. Zbl 1128.22010 MR 2327310

[Phi] N. C. Phillips, K-theory for Fréchet algebras. Internat. J. Math. 2 (1991), 77-129. Zbl 0744.46065 MR 1082838

[Ply] R. J. Plymen, Reduced $C^{*}$-algebra for reductive $p$-adic groups. J. Funct. Anal. 88 (1990), 251-266. Zbl 0718.22003 MR 1038441

[Sch] P. Schneider, The cyclic homology of $p$-adic reductive groups. J. Reine Angew. Math. 475 (1996), 39-54. Zbl 0849.22007 MR 1396725

[ScSt] P. Schneider and U. Stuhler, Representation theory and sheaves on the Bruhat-Tits building. Inst. Hautes Études Sci. Publ. Math. 85 (1997), 97-191. Zbl 0892.22012 MR 1471867

[SSZ] P. Schneider and E.-W. Zink, $K$-types for the tempered components of a $p$-adic general linear group. With an Appendix: The definition of the tempered category by P. Schneider and U. Stuhler. J. Reine Angew. Math. 517 (1999), 161-208. Zbl 0934.22021 MR 1728541 
[ScZi] P. Schneider and E.-W. Zink, The algebraic theory of tempered representations of p-adic groups I. Parabolic induction and restriction. J. Inst. Math. Jussieu 6 (2007), 639-688. Zbl 1126.22008 MR 2337311

[Sil] A. J. Silberger, Introduction to harmonic analysis on reductive p-adic groups. Math. Notes 23, Princeton University Press, Princeton, N.J., 1979. Zbl 0458.22006 MR 0544991

[Sol1] M. Solleveld, Some Fréchet algebras for which the Chern character is an isomorphism. K-Theory 36 (2005), 275-290 (2006). Zbl 1113.19002 MR 2275008

[Sol2] M. Solleveld, Periodic cyclic homology of Hecke algebras and their Schwartz completions. Preprint 2006. arXiv:math.KT/0605042

[Sol3] M. Solleveld Periodic cyclic homology of affine Hecke algebras., Ph.D. Thesis, Universiteit van Amsterdam, Amsterdam 2007.

[Tits] J. Tits, Reductive groups over local fields. In Automorphic forms, representations and L-functions (Proc. Sympos. Pure Math., Oregon State Univ., Corvallis, Ore., 1977), Part 1, Proc. Sympos. Pure Math. 33, Amer. Math. Soc., Providence, R.I., 1979, 29-69. Zbl 0415.20035 MR 0546588

[Tou] J.-C. Tougeron, Idéaux de fonctions différentiables. Ergeb. Math. Grenzgeb. 71, Springer-Verlag, Berlin 1972. Zbl 0251.58001 MR 0440598

[Vig] M.-F. Vignéras, On formal dimensions for reductive $p$-adic groups. In Festschrift in honor of I. I. Piatetski-Shapiro on the occasion of his sixtieth birthday (Ramat Aviv, 1989), Part I, Israel Math. Conf. Proc. 2, Weizmann, Jerusalem 1990, 225-266. Zbl 0732.22007 MR 1159104

[Voi1] C. Voigt, A new description of equivariant cohomology for totally disconnected groups. J. K-Theory 1 (2008), 431-472. Zbl 1162.19003 MR 2433277

[Voi2] C. Voigt, Equivariant local cyclic homology and the equivariant Chern-Connes character. Doc. Math. 12 (2007), 313-359. Zbl 1124.19002 MR 2365905

[Voi3] C. Voigt, Chern character for totally disconnected groups. Math. Ann. 343 (2009), 507-540. Zbl 1163.46045 MR 2480702

[Wal] J.-L. Waldspurger, La formule de Plancherel pour les groupes $p$-adiques (d'après Harish-Chandra). J. Inst. Math. Jussieu 2 (2003), 235-333. Zbl 1029.22016 MR 1989693

[Was] A. Wassermann, Cyclic cohomology of algebras of smooth functions on orbifolds. In Operator algebras and applications, Vol. 1, London Math. Soc. Lecture Note Ser. 135, Cambridge Univ. Press, Cambridge 1988, 229-244. Zbl 0693.55015 MR 0996447

[Wod] M. Wodzicki, The long exact sequence in cyclic homology associated with an extension of algebras. C. R. Acad. Sci. Paris Sér. I Math. 306 (1988), 399-403. Zbl 0637.16014 MR 934604

Received November 7, 2007; revised August 27, 2008

M. Solleveld, Mathematisches Institut, Georg-August-Universität Göttingen,

Bunsenstraße 3-5, 37073 Göttingen, Germany

E-mail: Maarten.Solleveld@mathematik.uni-goettingen.de 